
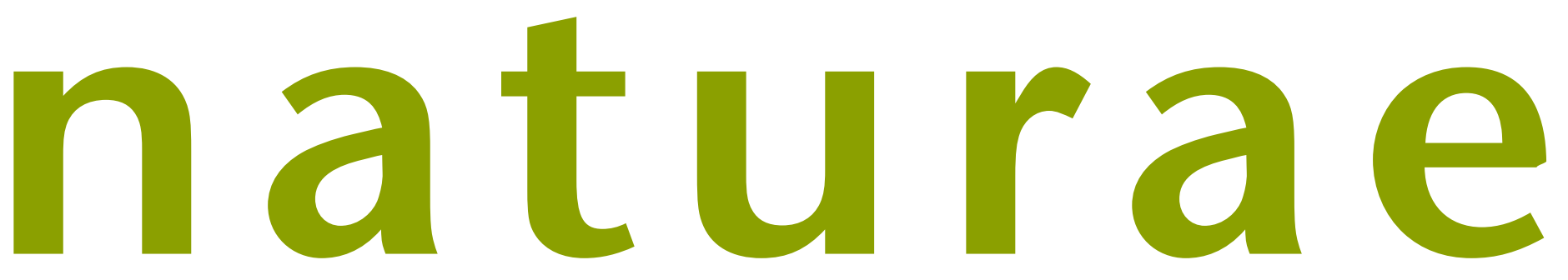

$2021 \cdot 14$

\title{
Priorités régionales de conservation pour les Mollusques continentaux:
éfi pour les taxons mal documentés un défi pour les taxons mal documentés
}

Jean-Michel BICHAIN, Kevin UMBRECHT, Julien RYELANDT \& Xavier CUCHERAT

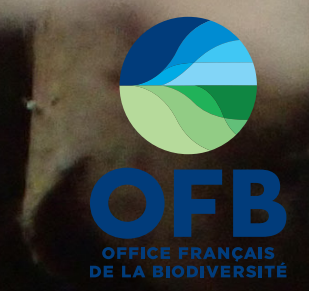


DiRECTEUR DE LA PUBLICATION / PUBLICATION DIRECTOR: Bruno David,

Président du Muséum national d'Histoire naturelle

RÉDACTEUR EN CHEF / EDITOR-IN-CHIEF: Jean-Philippe Siblet

ASSISTANTE DE RÉDACTION / ASSISTANT EDITOR: Sarah Figuet (naturae@mnhn.fr)

Mise EN PAGE / PAGE LAYOUT: Sarah Figuet

COMITÉ SCIENTIFIQUE / SCIENTIFIC BOARD:

Luc Abbadie (UPMC, Paris)

Luc Barbier (Parc naturel régional des caps et marais d'Opale, Colembert)

Aurélien Besnard (CEFE, Montpellier)

Vincent Boullet (Expert indépendant flore/végétation, Frugières-le-Pin)

Hervé Brustel (École d'ingénieurs de Purpan, Toulouse)

Patrick De Wever (MNHN, Paris)

Thierry Dutoit (UMR CNRS IMBE, Avignon)

Éric Feunteun (MNHN, Dinard)

Romain Garrouste (MNHN, Paris)

Grégoire Gautier (DRAAF Occitanie, Toulouse)

Olivier Gilg (Réserves naturelles de France, Dijon)

Frédéric Gosselin (Irstea, Nogent-sur-Vernisson)

Patrick Haffner (UMS PatriNat, Paris)

Frédéric Hendoux (MNHN, Paris)

Xavier Houard (OPIE, Guyancourt)

Isabelle Leviol (MNHN, Concarneau)

Francis Meunier (Conservatoire d'espaces naturels - Hauts-de-France, Amiens)

Serge Muller (MNHN, Paris)

Francis Olivereau (DREAL Centre, Orléans)

Laurent Poncet (UMS PatriNat, Paris)

Nicolas Poulet (OFB, Vincennes)

Jean-Philippe Siblet (UMS PatriNat, Paris)

Laurent Tillon (ONF, Paris)

Julien Touroult (UMS PatriNat, Paris)

COUVERTURE / COVER:

Cochlodina orthostoma (Menke, 1828) à proximité de Saint-Hippolyte (Doubs). Crédit photo: Julien Ryelandt.

Naturae est une revue en flux continu publiée par les Publications scientifiques du Muséum, Paris

Naturae is a fast track journal published by the Museum Science Press, Paris

Les Publications scientifiques du Muséum publient aussi / The Museum Science Press also publish:

Adansonia, Zoosystema, Anthropozoologica, European Journal of Taxonomy, Geodiversitas, Cryptogamie sous-sections Algologie, Bryologie, Mycologie, Comptes Rendus Palevol.

Diffusion - Publications scientifiques Muséum national d'Histoire naturelle

CP $41-57$ rue Cuvier F-75231 Paris cedex 05 (France)

Tél. : 33 (0)1 40794805 / Fax: 33 (0)1 40793840

diff.pub@mnhn.fr / http://sciencepress.mnhn.fr

(C) Publications scientifiques du Muséum national d'Histoire naturelle, Paris, 2021

ISSN (électronique / electronic): 1638-9387 


\title{
Priorités régionales de conservation pour les Mollusques continentaux: un défi pour les taxons mal documentés
}

\author{
Jean-Michel BICHAIN \\ Kevin UMBRECHT \\ Musée d'Histoire naturelle et d'Ethnographie de Colmar, \\ 11 rue de Turenne, F-68000 Colmar (France) \\ jean-michel.bichain@museumcolmar.org \\ kevin.umbrecht@museumcolmar.org \\ Julien RYELANDT \\ Conservatoire botanique national de Franche Comté - Observatoire régional des Invertébrés, \\ 7 rue Voirin, F-25000 Besançon (France) \\ julien.ryelandt.ori@cbnfc.org \\ Xavier CUCHERAT \\ arion.idé, 10 rue Louis Aragon, F-59147 Gondecourt (France) \\ xavier.cucherat@wanadoo.fr \\ Soumis le 30 octobre 2020 | Accepté le 18 janvier 2021 | Publié le 23 juin 2021
}

MOTS CLÉS

Hiérarchisation, méthode de notation

par point

Bourgogne-Franche-

Comté,

Gastropoda

Bivalvia.

Bichain J.-M., Umbrecht K., Ryelandt J. \& Cucherat X. 2021. - Priorités régionales de conservation pour les Mollusques continentaux: un défi pour les taxons mal documentés. Naturae 2021 (14): 183-211. https://doi.org/10.5852/naturae2021a14

\section{RÉSUMÉ}

À peine $1 \%$ des Mollusques continentaux, à l'échelle européenne ou nationale, bénéficient d'un statut réglementaire de protection bien que près de $30 \%$ à $40 \%$ des espèces du groupe soient considérées comme menacées d'après les critères de l'UICN. Il paraît donc nécessaire de fournir des outils complémentaires pour la gestion/planification des stratégies régionales de conservation. Nous proposons ici une liste hiérarchisée des espèces à enjeux de conservation à partir d'une méthode de notation par points fondée sur trois critères et d'un indice concernant l'état des connaissances. Le jeu de données mobilise environ 60000 données d'occurrence pour 227 espèces cibles pour les régions Grand Est et Bourgogne-Franche-Comté. Nous observons des disparités importantes de quantité/qualité d'informations non seulement entre les sous-unités géographiques, mais aussi entre les différents grands groupes taxonomiques. Les biais taxonomiques s'expliquent notamment par la rareté intrinsèque des espèces, la difficulté d'identifier les taxons et/ou la fiabilité de leurs descripteurs et les mesures réglementaires contraignantes qui orientent la recherche des espèces. Le résultat de la procédure de hiérarchisation indique que $51 \%$ des espèces cibles, dont $15 \%$ présentent un risque majeur d'extinction à l'échelle globale ou régionale, présentent un volume et une qualité d'informations jugés satisfaisants afin d'évaluer leur niveau de priorisation. Les autres espèces présentent un handicap de connaissance taxonomique, d'identification et/ou de répartition qui ne permet pas d'évaluer rigoureusement leur degré de priorisation. Nous montrons donc qu'il n'existe pas de bons indicateurs, dans l'état actuel des connaissances, pour le législateur et/ou le conservateur afin d'identifier, évaluer et prioriser les besoins de conservation des Mollusques à l'échelle régionale. Nos résultats permettent en revanche d'établir les bases d'une stratégie régionale en séparant les réels enjeux de conservation des enjeux d'amélioration des connaissances. 


\author{
KEY WORDS \\ Prioritisation \\ point-scoring method, \\ Grand Est, \\ Bourgogne-Franche- \\ Comté, \\ Gastropoda, \\ Bivalvia.
}

\begin{abstract}
Regional conservation priorities for non-marine Mollusca: a challenge for poorly-documented taxa. Barely $1 \%$ of continental molluscs, on a European or French national scale, have a protection status, although around $30 \%$ to $40 \%$ of the species are considered threatened according to the IUCN criteria. It therefore appears necessary to provide complementary tools for the management/planning of regional conservation strategies. The current study proposes a hierarchical list of species with conservation stakes built on a point-scoring method based on three criteria and an index linked to the current state of knowledge. Our dataset contains around 60000 occurrence data for 227 target species for the Grand Est and Bourgogne-Franche-Comté, two administrative regions in eastern France. We observe significant disparities in the quantity/quality of available information not only between geographical sub-units but also among major taxonomic groups. The taxonomic biases can be explained by the intrinsic rarity of species, the difficulty in identifying taxa and/or the reliability of their descriptors, and the restrictive protection measures that unbalance the search for species in the field. The result of the prioritisation procedure indicates that $51 \%$ of the target species, $15 \%$ of which present a major risk of global or regional extinction, present a volume and quality of information deemed satisfactory in order to assess their level of prioritisation. The other species present a handicap in terms of taxonomic knowledge, identification and/or distribution that makes it impossible to rigorously assess their conservation priority. We therefore show that there is no good indicator - in the current state of knowledge - for the legislator and/or the curator to identify, evaluate and prioritise regional molluscs conservation plans. Our results do, however, make it possible to establish the basis for a regional strategy by separating real conservation issues from issues of improving knowledge.
\end{abstract}

\section{INTRODUCTION}

La possibilité d'une sixième extinction de masse, c'est-àdire une perte massive, globale et rapide de la biodiversité mondiale (Leakey \& Lewin 1996; Pimm et al. 2014), liée à l'impact des activités humaines a émergé concomitamment avec le changement de paradigme concernant l'incomplétude de nos connaissances sur la réelle diversité biologique de la planète (Erwin 1982; Stork 2018). Il est désormais admis que les quelques deux millions d'espèces aujourd'hui décrites (Chapman 2009) ne représenteraient qu'une faible fraction du nombre global des espèces (Mora et al. 2011; Locey \& Lennon 2016; Larsen et al. 2017). Avec un volume de descriptions de l'ordre de 15000 à 20000 nouvelles espèces chaque année, plusieurs siècles seront théoriquement nécessaires afin d'achever l'inventaire du vivant. En d'autres termes, le monde que nous voulons explorer et protéger nous est largement inconnu. Dans ce double contexte, la communauté scientifique produit un nombre considérable de publications qui tente non seulement de décrire le vivant dans ses multiples dimensions, mais aussi - à travers une approche apparentée pour certains à de la médecine urgentiste (Bottrill et al. 2008; Wilson \& Law 2016; se reporter à Vucetich et al. 2017 pour une opinion opposée) - de proposer concepts, stratégies et solutions afin de ralentir voire d'enrayer la disparition/dégradation des milieux naturels (Godet \& Devictor 2018).

Cependant, la réussite et la pertinence des actions de conservation, fondées sur l'évaluation et la priorisation des risques, sont directement liées au volume, à la qualité des informations mobilisées et à leur transfert/traduction vers les domaines réservés à la législation, à la gestion, à la médiation scientifique et au génie écologique. Or, il apparaît que de nombreux biais existent et persistent (Di Marco et al. 2017) dans et entre les produits de la recherche et, par voie de conséquence, fausse non seulement notre regard collectif sur les réels enjeux de conservation, mais aussi sur l'orientation que devrait prendre les actions en faveur des milieux naturels.

En effet, la surreprésentation d'une infime part de la biodiversité, essentiellement les Oiseaux et les Mammifères, comme sujet et objet d'étude, constitue l'un de ces biais nommé par ailleurs biais taxonomique (Bonnet et al. 2002; Clark \& May 2002). En d'autres termes, à peine $1 \%$ de la biodiversité planétaire polarise et/ou capte la très grande majorité de l'attention et des ressources financières dédiées à la connaissance et à la préservation des espèces et des milieux naturels (Cardoso et al. 2011 a ; Donaldson et al. 2016; Mammides 2019; Eisenhauer et al. 2019). L'angle mort est plutôt large. D'autre part, les acteurs de la recherche sont rarement ceux qui définissent, gèrent et mettent en ouvre les plans de conservation et la disjonction entre ces deux compartiments recherche/gestion est un phénomène réel (Knight et al. 2008; Game et al. 2013). Ces déséquilibres de connaissances et de moyens expliquent en partie la difficulté d'atteindre les différents objectifs fixés depuis 1992 par la Convention sur la Diversité biologique et/ou par certaines de leurs déclinaisons européennes (voir par exemple Tittensor et al. 2014).

Les Mollusques continentaux illustrent cet état de fait. Ils appartiennent au phylum animal le plus diversifié, après les Arthropodes, avec $70000-76000$ espèces décrites (Rosenberg 2014). Ils incluent deux grandes lignées évolutives distinctes avec les classes des Bivalvia (Bivalves ou moules) et des Gastropoda (Escargots terrestres et aquatiques, Limaces). Le nombre connu d'espèces continentales est d'environ 24000 (Lydeard et al. 2004; Rosenberg 2014) et de 5100 (Strong et al. 2008; 
Graf 2013; Rosenberg 2014) respectivement dans les habitats terrestres et aquatiques. En revanche, la diversité spécifique réelle pourrait atteindre 40000 espèces dans les habitats terrestres et 10000 dans les habitats aquatiques (Lydeard et al. 2004). Concernant les menaces, les Mollusques cumulent le plus grand nombre d'extinctions enregistrées, bien que seules 8,5-10 \% des espèces du groupe soient évaluées selon la méthodologie UICN, dont 30 à $40 \%$ placés dans la catégorie Data Deficient (Cowie et al. 2017; Böhm et al. 2020). Ce fort taux de données insuffisantes, qui n'est que de l'ordre de $5 \%$ pour les Oiseaux et les Mammifères, s'explique par la pauvreté des données pertinentes disponibles pour les Mollusques, et les Invertébrés d'une manière générale (Cardoso et al. 2011b, 2012), qui peuvent satisfaire les critères de catégorisation UICN. En effet, à l'échelle du groupe, une grande part des espèces est connue uniquement sur la base des informations primaires contenues dans leur description (Régnier et al. 2015). Pour l'autre part, outre les problématiques taxonomiques de délimitation des espèces, il faut bien convenir que peu de données documentent leur biologie, écologie, dynamique des populations ou aire de répartition. En conséquence, la première Liste rouge des des Mollusques de France a vu le jour récemment (UICN Comité français et al. sous presse), alors que l'ensemble des vertébrés du territoire métropolitain en sont à leur deuxième génération d'évaluation UICN.

Enfin, au niveau européen, une trentaine de taxons terminaux et leur(s) habitat(s) sont réglementés par les annexes II, IV et $\mathrm{V}$ de la Directive de l'Union européenne 92/43/CEE et 59 sur le territoire métropolitain via les articles 2, 3 et 4 de l'arrêté du 23 avril 2007 fixant les listes des Mollusques protégés sur l'ensemble du territoire et les modalités de leur protection. Cela représente donc moins de $1 \%$ des 3400 espèces en Europe (Cuttelod et al. 2011) et environ $7 \%$ des 754 taxons terminaux présents en France métropolitaine (Gargominy et al. 2011). Nous sommes donc très loin d'une prise en compte réglementaire de la hauteur des menaces mis en évidence par les experts du groupe (Régnier et al. 2009; Cuttelod et al. 2011; Cowie et al. 2017; Böhm et al. 2020). Il s'agit de fait des seuls outils dont disposent les autorités environnementales, ainsi que les gestionnaires des espèces/espaces naturels pour la prise en compte spécifique des Mollusques continentaux.

L'urgence est donc de fournir, en l'état actuel des connaissances, des outils complémentaires qui puissent orienter de manière pertinente la gouvernance et la gestion des stratégies de protection et de conservation. Il est généralement admis que trois étapes sont nécessaires et ce, afin de mieux répartir les ressources disponibles vers les cibles (taxons, populations, habitats, lieux) les plus vulnérables (Pullin et al. 2013) :

- prioriser les cibles à partir d'une évaluation des menaces réelles et/ou potentielles;

- hiérarchiser les projets et les actions de conservation;

- sélectionner et mettre en œuvre des actions de conservation puis évaluer leur succès.

Les méthodes de classification s'insèrent dans cette stratégie globale en proposant notamment des listes d'espèces hiérarchisées en fonction des degrés de menace et en tenant compte de la disponibilité des données (voir Le Berre et al. 2019 pour une vue d'ensemble). Ces approches critériées et reproductibles different des listes de protection réglementaire qui reposent habituellement sur des avis d'experts et non sur des critères évalués objectivement. Par ailleurs, bien que les Listes rouges soient des outils essentiels pour la définition des priorités des actions de conservation, elles ne reflètent pas toujours les enjeux réels, en particulier pour une zone géographique donnée dans laquelle il peut être préférable de concentrer les efforts sur les espèces (sub)endémiques, ou en marge d'aire de répartition, pour lesquelles la responsabilité régionale est élevée.

Dans ce cadre, il nous a semblé pertinent - en première étape - de proposer une telle démarche de classification, à l'échelle des deux grandes régions Grand Est et BourgogneFranche-Comté, afin d'initier des priorités régionales de conservation pour les Mollusques continentaux. Les objectifs de cet article sont donc de fournir:

- une évaluation de la quantité et de la qualité des informations disponibles et la hauteur des différents biais d'observation;

- une liste hiérarchisée des espèces à enjeux de conservation fondée sur la méthode de Gauthier et al. (2010) et des grands axes d'action de conservation les concernant;

- une liste des taxons pour lesquels il est nécessaire d'améliorer prioritairement les connaissances afin de mieux déterminer leurs réels enjeux de conservation.

\section{MATÉRIEL ET MÉTHODES}

\section{AIRE D'ÉTUDE}

La zone d'étude couvre une superficie de $105488 \mathrm{~km}^{2} \mathrm{du}$ nord-est de la France, soit approximativement $1 / 6$ du territoire national. Elle est sous l'autorité administrative de deux grandes régions, le Grand Est avec $57704 \mathrm{~km}^{2}$ et la Bourgogne-FrancheComté avec $47784 \mathrm{~km}^{2}$, lesquelles incluent respectivement six et trois Parc naturels régionaux (PNR), et un projet de parc national à cheval sur les deux régions. Ce découpage territorial n'est pas uniforme d'un point de vue biogéographique par sa situation de zone de transition entre les influences océanique, continentale et montagnarde. Il inclut, par ailleurs, plusieurs grandes régions naturelles dont, à l'intérieur des frontières françaises, l'ensemble ou grande partie des massifs des Vosges, des Ardennes et du Jura, ainsi que la plaine de Champagne, du plateau Lorrain et de Langres, des fossés rhénan et bressan mais aussi la bordure nord-est du Massif central. Les altitudes s'étalent de 60 mètres en plaine de Champagne jusqu'à 1424 et 1495 mètres respectivement dans les Vosges et le Jura. Les limites de la zone d'étude sont donc suffisamment larges afin d'y inclure une mosaïque d'unités biogéographiques, mais aussi un zonage administratif, clairement identifié, de gestion des espaces naturels.

\section{ESPÈCES CIBLES}

Le niveau taxonomique ici considéré est au rang de l'espèce uniquement. Les espèces documentées comme anthropophiles, cryptogènes (Carlton 1996), introduites ou introduites envahissantes à l'échelle nationale (Gargominy et al. 2019) ou régionale (Bichain et al. 2019) ont été écartées de la liste 
TABLEAU 1. - Notation par critère. La note finale de 1 à 5 pour le critère de Vulnérabilité a été calculée par la formule suivante: $2 \times$ (note sur 5 de la catégorisation mondiale) + (note sur 5 de la catégorisation européenne)/3. Abréviations: CR, en danger critique d'extinction; DD, données insuffisantes; EN, en danger; NE, non évaluée; Nsp, nombre d'espèces par classe de note de 1 à 5 ; Nsp*, le premier chiffre indique le nombre d'espèces par classe de note de 1 à 5 pour la catégorisation mondiale, le second pour la catégorisation européenne et entre parenthèses par classe de note globale arrondie; NT, quasi menacée; VU, vulnérable. ** Les habitats ici considérés comme vulnérables (c'est-à-dire sous forte pression anthropique) sont les prairies/pelouses xérothermes, les habitats alluviaux inondables, les tourbières alcalines, les prairies d'altitude et les milieux aquatiques oligotrophes.

\begin{tabular}{|c|c|c|c|c|c|c|}
\hline \multirow[b]{2}{*}{ Note } & \multicolumn{2}{|c|}{ Responsabilité Régionale (RR) } & \multicolumn{2}{|l|}{ Vulnérabilité (V) } & \multicolumn{2}{|l|}{ Rareté locale (R) } \\
\hline & $\begin{array}{l}\text { Distribution biogéographique } \\
\text { (d'après TaxRef v13.0 } \\
\text { et littérature disponible) }\end{array}$ & Nsp & $\begin{array}{l}\text { Catégories de menace (d'après } \\
\text { les Listes rouges UICN } \\
\text { mondiales et européennes) }\end{array}$ & Nsp* & $\begin{array}{l}\text { Nombre de mailles } \\
2 \times 2 \mathrm{~km} \text { occupées } \\
\text { (d'après la base } \\
\text { de données globale) }\end{array}$ & Nsp \\
\hline 5 & Micro-endémique & 8 & CR & $0 / 1(0)$ & {$[1-8[$} & 49 \\
\hline 4 & Endémique & 14 & EN & $6 / 3(6)$ & [9-37[ & 42 \\
\hline 3 & Subendémique & 3 & VU & $6 / 6(9)$ & {$[38-96[$} & 45 \\
\hline 2 & $\begin{array}{l}\text { Extrême limite occidentale de } \\
\text { répartition ou répartition } \\
\text { française strictement limitée } \\
\text { à la zone d'étude) }\end{array}$ & 30 & $\begin{array}{l}\text { NT (+0,5 ou }+1 \text { si habitats évalués } \\
\text { par nous comme vulnérables }{ }^{\star \star} \\
\text { ou déclin régional) }\end{array}$ & $6 / 11(22)$ & {$[97-201[$} & 45 \\
\hline 1 & $\begin{array}{l}\text { Répartition plus large (+1 si taxon } \\
\text { rare à l'échelle de son aire } \\
\text { globale de répartition; +0,5 si } \\
\text { en limite d'aire ou répartition } \\
\text { fragmentée/disjointe) }\end{array}$ & 172 & $\begin{array}{l}\text { NE ou DD (+0,5 ou }+1 \text { si habitats } \\
\text { évalués comme vulnérables** } \\
\text { ou déclin régional) }\end{array}$ & 209/206 (190) & [202-865] & 46 \\
\hline
\end{tabular}

des espèces ciblées par les enjeux de conservation. Les espèces qui ne présentent pas de donnée postérieure à 1977 ne sont pas catégorisées, mais traitées à part comme potentiellement éteintes à l'échelle régionale.

\section{ÉLABORATION DU CORPUS GLOBAL DE DONNÉES}

La base de données initiale a été compilée à partir de l'ensemble des données d'occurrence "Gastéropodes» et "Bivalves » continentaux, transmises par l'Inventaire national du Patrimoine naturel (INPN) (plateforme nationale du Système d'Information de l'Inventaire du Patrimoine naturel [SINP]) le 9 janvier 2020 (dossier no2370), en provenance des deux grandes régions Grand Est et Bourgogne-Franche-Comté. Elle a été complétée par des données bibliographiques, muséales, d'experts et/ou de structures associatives dédiées à la biodiversité. Elle couvre la période de 1800 à janvier 2020.

Un premier traitement a consisté à écarter les données d'observation ne faisant pas référence explicitement à un nom de récolteur, à une date et à un lieu de récolte, dont la précision est au moins à l'année, à la commune, au lieu-dit ou au point. De la même manière, les données ne se rapportant pas à un binom latin ou pour lesquelles la détermination a semblé douteuse, inadéquate ou fausse n'ont pas été retenues. La liste d'espèces retenues pour le Grand Est est celle de Bichain et al. (2019). La liste de Bourgogne-France-Comté a, quant à elle, été construite pour le présent travail sur la base de la méthodologie mise en ouvre par Bichain et al. (2019).

Dans une seconde étape, afin d'éviter d'inclure des erreurs probables de détermination par les observateurs les moins expérimentés, nous avons écarté toutes les données pour lesquelles la détermination ne semble pas satisfaire aux exigences diagnostiques proposées par Bichain et al. (2019). Nous avons donc attribué à chaque observateur/déterminateur un niveau d'expertise faible, moyen, fort ou inconnu. Les données issues d'un protocole $\mathrm{ADNe}$ sont ici catégorisées comme niveau d'expertise fort. Conséquemment, nous avons éliminé les données provenant des niveaux d'expertise faible et moyen pour les espèces catégorisées respectivement de N3 à N5 et de N4 à N5 par Bichain et al. (2019: tableau 1). En effet, ces catégories correspondent à des niveaux de difficultés de détermination qui nécessitent le recourt à des caractères conchyliologiques seulement (N1 à N3), et/ou à des caractères diagnostiques morpho-anatomiques (N3+ et N4) et/ou génétiques (N5).

Enfin, une troisième étape a consisté à éliminer les données antérieures à 1977, afin de minimiser la hauteur des changements écologiques potentiels qui ont pu s'opérer dans les milieux naturels, l'impact des changements de paradigme taxonomique et la dégradation des informations liées aux données anciennes (Tessarolo et al. 2017). Ce choix de 1977 est un compromis entre volume de perte d'informations et statut de «donnée ancienne» généralement accepté sur l'intervalle 30-50 ans.

Cette série de traitements, résumée dans la Figure 1, permet de réduire la part des informations jugées imprécises, inadéquates ou erronées. La base de données résultante fournit pour chaque espèce: l'année de première (Y1) et de dernière (Y2) observation, le nombre total d'observations $\left(\mathrm{N}_{\mathrm{obs}}\right)$ et d'occurrences $\left(\mathrm{N}_{\mathrm{occ}}\right)$. $\mathrm{N}_{\mathrm{obs}}$ correspond à l'ensemble des citations géographiques ayant des observateurs/récolteurs distincts et/ou des dates d'observation différentes $(\mathrm{Y} 1 \neq \mathrm{Y} 2) . \mathrm{N}_{\mathrm{occ}}$ correspond à des données d'observation spatialement distinctes sur une grille de $2 \times 2 \mathrm{~km}$ généré à partir du logiciel QGIS 3.12 (http://qgis.osgeo.org, dernière consultation le 30 octobre 2020).

\section{BIAIS GÉOGRAPHIQUES ET TAXONOMIQUES}

L'évaluation des biais potentiels d'occurrences est réalisée sur le jeu de données final. Les biais géographiques, entre les subdivisions administratives (départements, régions) de la zone d'étude, sont estimés en comparant le nombre total 


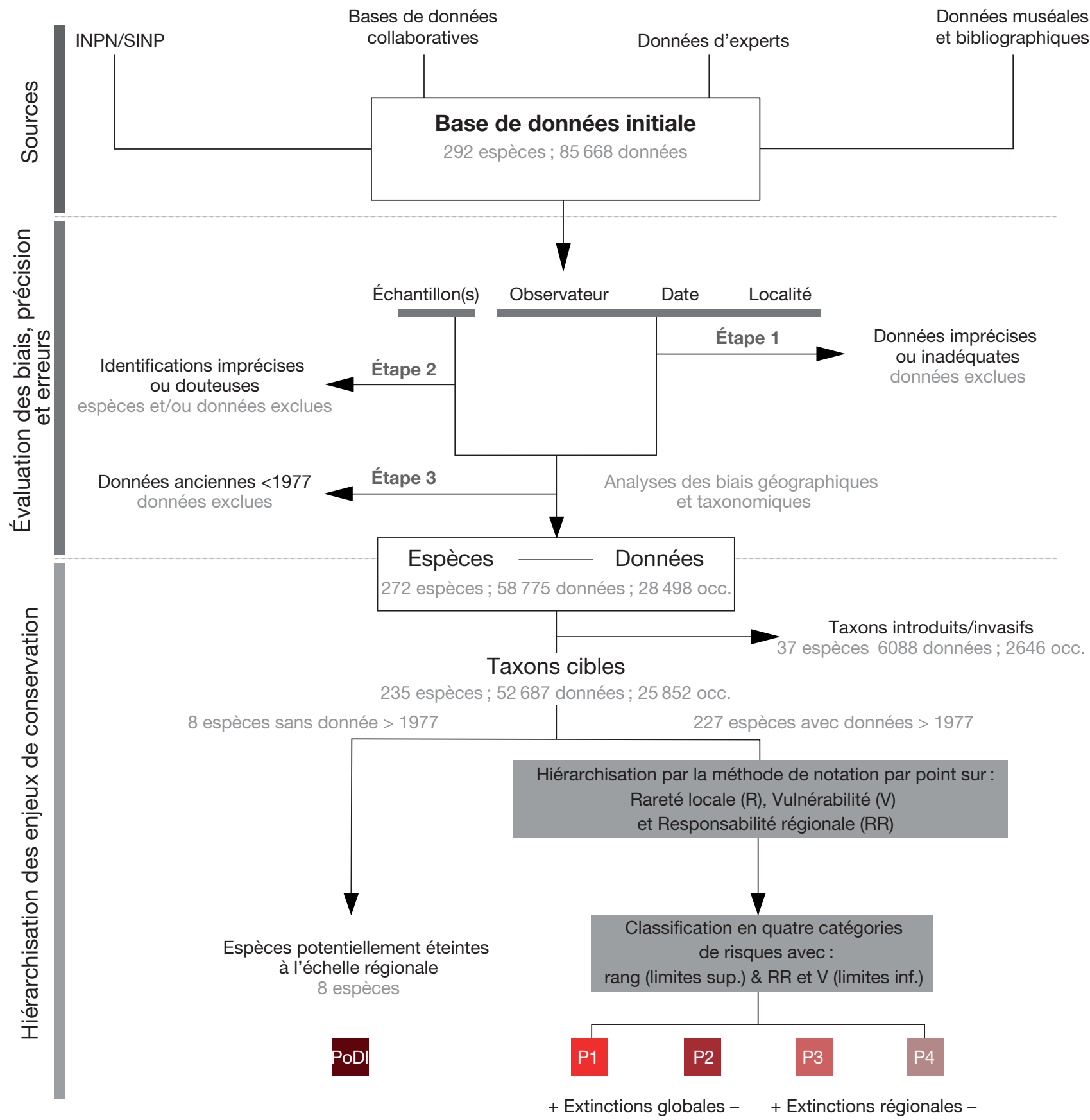

FIG. 1. - Vue d'ensemble de la procédure de traitement des données et de hiérarchisation des enjeux de conservation. Abréviations: occ., occurrences; P1, risque élevé d'extinction globale ou régionale; $\mathbf{P 2}$, risque d'extinction globale ou régionale; $\mathbf{P 3}$, risque de réduction plus ou moins significative d'aire d'occurrence pouvant conduire à des extinctions régionales; $\mathbf{P 4}$, risque de déclin ne conduisant pas à des extinctions régionales à court ou moyen terme; PoDi, potentiellement disparu dans la zone d'étude.

d'observations $\mathrm{N}_{\text {obs }}$, d'occurrences $\mathrm{N}_{\text {occ }}$, de mailles échantillonnées $\mathrm{N}_{\text {maille }}$ et le nombre moyen d'observations par maille

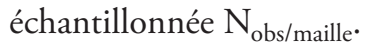

L'effort global d'observation pour chaque subdivision administrative est évalué à travers quatre paramètres:

- le nombre d'observations par unité de surface $\left(\mathrm{N}_{\mathrm{obs}} / \mathrm{St}\right)$ où St est la surface totale du territoire considéré;

- la proportion de mailles documentées avec au moins une donnée d'observation $\left(\mathrm{N}_{\text {maille }} / \mathrm{N}_{\mathrm{mt}}\right)$ où $\mathrm{N}_{\mathrm{mt}}$ est le nombre total de mailles dans le territoire considéré;
- la proportion de données expertes $\mathrm{N}_{\text {exp }}$

- le nombre moyen d'observations par maille documentée $\left(\mathrm{N}_{\text {obs }} / \mathrm{N}_{\text {maille }}\right)$.

La méthode des quartiles est appliquée, afin d'attribuer un score de 1 à 4 à chacun des paramètres par département. Chaque département obtient une note globale, de 4 à 16, par sommation des différents scores. Le score le plus haut représente l'effort d'observation le plus faible. Les notes globales sont discrétisées en quatre classes de taille égale signifiant un effort global d'observation allant de fort, moyen, faible à très faible. 
Par ailleurs, nous évaluons la complétude de l'inventaire taxonomique pour chaque département à travers le nombre d'espèces estimé $\mathrm{N}_{\mathrm{sp}(\mathrm{e})}$ calculé avec le logiciel EstimateS 9.1.0 (Colwell et al. 2012) via l'estimateur Chao2 avec son écart-type et intervalle de confiance à $95 \%$. Pour ce faire, les matrices de présence/absence des espèces par maille ont été implémentées dans EstimateS 9.1.0 avec le paramétrage par défaut et Chao 2 corrigé.

Nous estimons les biais taxonomiques en suivant la méthode proposée par Troudet et al. (2017). Nous avons donc calculé comment chaque groupe taxonomique s'écarte d'un échantillonnage idéal (I) pour lequel il serait échantillonné $\left(\mathrm{N}_{\mathrm{occ}}\right)$ de manière proportionnelle à son nombre d'espèces $\left(\mathrm{N}_{\mathrm{sp}}\right)$ avec $\mathrm{I}=\mathrm{N}_{\text {occ }}\left(\mathrm{N}_{\mathrm{sp}} / \mathrm{N}_{\text {tot }}\right)$ où $\mathrm{N}_{\text {tot }}$ est le nombre total d'espèces.

Ce calcul est appliqué à six partitions taxonomiques construites sur la base de divergences phylogénétiques profondes (Bivalves vs Gastéropodes; Hydrobies vs autres Gastéropodes d'eau douce), de macro-habitats (Gastéropodes terrestres vs Gastéropodes d'eau douce), d'un corps non pourvu d'une coquille externe (Limaces vs Escargots) et sur la taille globale des coquilles (Micro, Méso ou Macro-Bivalves). Bien que ces partitions ne représentent pas toutes des groupes monophylétiques, elles forment des entités classiquement traitées dans les ouvrages spécialisés de malacologie (Killeen et al. 2004; Kerney et al. 2006 ; Sysoev \& Schileyko 2009 ; Rowson et al. 2014; Gloër 2019).

Au final, l'estimation de ces biais doit permettre de proposer des stratégies adaptées et ciblées pour l'amélioration des connaissances chorologiques ou sur des groupes taxonomiques, en rapport avec la priorisation des enjeux de conservation.

\section{HIÉRARCHISATION DES ENJEUX DE CONSERVATION :}

\section{CRITÈRES ET MÉTHODE}

La méthode de hiérarchisation est adaptée de la méthode de notation par point (point-scoring) de Gauthier et al. (2010). Les trois critères utilisés sont la responsabilité régionale (RR), la vulnérabilité $(\mathrm{V})$ et la rareté locale $(\mathrm{R})$. Pour chacun de ces critères, une note de 1 à 5 est attribuée à chaque taxon, cinq représentant le score le plus haut (Tableau 1).

Le critère de responsabilité régionale $(\mathrm{RR})$ vise à donner la priorité aux taxons ayant une distribution mondiale restreinte ou en extrême limite occidentale d'aire de répartition. Le statut d'endémique ou de subendémique a été attribué en suivant le référentiel TaxRef v13.0 (Gargominy et al. 2019). Nous avons par ailleurs ajouté la catégorie de micro-endémique pour les espèces dont la répartition globale est limitée à une ou deux localités. Un taxon endémique est défini comme un taxon naturellement restreint à la zone géographique considérée. Un taxon subendémique est considéré comme naturellement restreint à une zone un peu plus grande que la zone géographique considérée, mais dont les principales populations se situent dans la zone géographique considérée.

Les taxons qui atteignent leur extrême marge occidentale de répartition européenne, et qui sont présents en France uniquement dans la zone d'étude, impliquent une responsabilité régionale quantifiée ici par un score de 2 .
Sur la base de la littérature spécialisée (Kerney et al. 2006 ; Welter-Schultes 2012 ; Prié 2017 ; Gloër 2019), nous attribuons aux taxons plus largement répandus au sein de la zone d'étude (score de 1) une majoration d'un demi-point pour ceux en marge d'aire de répartition ou ayant des aires de distributions fragmentées ou disjointes et d'un point pour les taxons rares ou peu fréquents à l'échelle de leur aire globale de répartition.

Le critère de vulnérabilité $(\mathrm{V})$ vise à donner la priorité aux taxons menacés d'extinction, à plus ou moins court terme, à l'échelle de leur aire globale de répartition. Ce critère est évalué à partir des catégories UICN proposées dans les Listes rouges mondiale et européenne. Une pondération en faveur de la catégorisation mondiale est ici appliquée (Tableau 1). Une majoration d'un demi-point ou d'un point est attribuée aux taxons qui sont documentés à travers la littérature comme en déclin/régression dans la région et/ou pour lesquels les habitats sont considérés comme vulnérables.

Le critère de rareté locale $(\mathrm{R})$ vise à donner la priorité aux taxons géographiquement rares dans la zone d'étude avec comme hypothèse sous-jacente qu'ils ont une plus grande probabilité d'extinction en raison de facteurs stochastiques (Gauthier et al. 2010). Ce critère est évalué à partir du nombre d'occurrences spatialement distinctes sur une grille de $2 \times 2 \mathrm{~km}$. Les coordonnées $\mathrm{X}$ et $\mathrm{Y}$ du centroïde des communes sont ici utilisées les cas échéants. La méthode des quantiles a été utilisée pour créer cinq classes de taille égale où la note la plus élevée est attribuée à la classe d'occurrences les plus faibles.

Le score global est obtenu par multiplication des scores, plutôt que par addition, afin de donner un poids relatif plus important aux taxons qui ont les plus hauts scores dans chacun des critères (Rodríguez et al. 2004). La hiérarchisation des taxons est réalisée par classement des scores finaux en ordre décroissant. Puis les taxons sont classés par scores décroissants obtenus pour chacun des critères: d'abord la responsabilité régionale, puis la vulnérabilité, et enfin la rareté locale.

À partir de ce classement, nous définissons par ailleurs quatre catégories indicatives de risques dont les bornes inférieures sont basées sur les scores des taxons pour les critères de la responsabilité régionale (RR) et secondairement de vulnérabilité (V) (Tableau 2).

La catégorie P1 est construite sur l'intervalle des plus hautes valeurs de score global qui permet d'inclure toutes les espèces pour lesquelles $\mathrm{RR}=5$. Les risques associés à cette catégorie sont la possible extinction globale ou régionale d'espèces (micro-)endémiques et/ou reconnues comme vulnérables. La catégorie P2 est construite sur les valeurs de score global et est bornée par P1, qui permet d'inclure toutes les espèces pour lesquelles $R R=3$, incluant de facto les espèces $R R=4$. Cette catégorie est indicative de risques d'extinction globale ou régionale d'espèces (sub)endémiques et/ou vulnérables. La catégorie P3 est construite sur les valeurs de score global et de classement et est bornée par P2, qui permet d'inclure toutes les espèces pour lesquelles $V \geq 3$. Cette catégorie est indicative de risques de réduction plus ou moins significa- 
TABLEAU 2. - Détermination des catégories d'enjeux de conservation et actions d'amélioration des connaissances. Abréviations: *, ces catégories ne doivent pas être interprétées comme mesure directe ou indirecte de la probabilité d'événement d'extinction et/de régression; $\mathbf{N}_{\mathbf{s p}}$, nombre d'espèces par classe de note de 1 à 5 .

\begin{tabular}{|c|c|c|c|c|c|}
\hline \multirow[b]{2}{*}{ Catégories indicatives de risque* } & \multirow{2}{*}{$\begin{array}{l}\text { Seuils } \\
\text { associés }\end{array}$} & \multicolumn{3}{|c|}{ Priorités de conservation } & \multirow[b]{2}{*}{ Actions } \\
\hline & & Rang & Score global & Nsp & \\
\hline $\begin{array}{l}\text { PoDi: potentiellement disparu dans la } \\
\text { zone d'étude. Le taxon n'a pas été } \\
\text { revu depuis } 1977\end{array}$ & - & - & - & 8 & $\begin{array}{l}\text { AC1. Mise en place prioritaire de la } \\
\text { recherche ciblée et extensive avec des } \\
\text { moyens adaptés (ADNe, cartographie } \\
\text { prédictive, etc.) }\end{array}$ \\
\hline $\begin{array}{l}\text { P1: risque élevé d'extinction globale } \\
\text { ou régionale }\end{array}$ & $\subseteq[\mathrm{RR}=5]$ & de 1 à 7 & $\begin{array}{l}25 \leq \mathrm{ScG} \\
\quad \leq 80\end{array}$ & 14 & \multirow{2}{*}{$\begin{array}{l}\text { AC2. Appliquer un programme de } \\
\text { cartographie, d'estimation et de suivi } \\
\text { des populations (répartition, effectifs, } \\
\text { dynamique) ainsi que l'évaluation des } \\
\text { pressions sur les habitats }\end{array}$} \\
\hline $\begin{array}{l}\text { P2: risque d'extinction globale ou } \\
\text { régionale }\end{array}$ & $\begin{array}{l}\mathrm{RR}<5 \\
\subseteq[\mathrm{RR}=3]\end{array}$ & de 8 à 21 & $\begin{array}{l}12 \leq \mathrm{ScG} \\
\quad<25\end{array}$ & 23 & \\
\hline $\begin{array}{l}\text { P3: risque de réduction plus ou moins } \\
\text { significative d'aire d'occurrence } \\
\text { pouvant conduire à des d'extinctions } \\
\text { régionales }\end{array}$ & $\begin{array}{l}\mathrm{RR}<3 \\
\subseteq[\mathrm{V} \geq 3]\end{array}$ & de 22 à 38 & $\begin{array}{c}3<\text { ScG }< \\
12\end{array}$ & 78 & $\begin{array}{l}\text { AC3. Favoriser la cartographie régionale } \\
\text { des populations et l'évaluation des } \\
\text { pressions globales sur les population/ } \\
\text { habitats }\end{array}$ \\
\hline $\begin{array}{l}\text { P4: risque de déclin ne conduisant pas } \\
\text { à des extinctions régionales à court } \\
\text { ou moyen terme }\end{array}$ & $\begin{array}{l}\mathrm{RR}<2 \\
\mathrm{~V}<3\end{array}$ & de 39 à 46 & $1 \leq \mathrm{ScG} \leq 3$ & 112 & $\begin{array}{l}\text { AC4. Améliorer la cartographie régionale } \\
\text { au moins en termes de présence/ } \\
\text { absence }\end{array}$ \\
\hline
\end{tabular}

tifs d'aire d'occurrence régionale et possiblement d'extinction d'espèces à l'échelle régionale. Le reste des taxons est catégorisé en $\mathrm{P} 4$ où les risques ne correspondent ni à des extinctions d'espèce, ni à des réductions d'aire d'occurrences pour des espèces identifiées comme vulnérables $(\mathrm{V} \geq 3)$ ou à forte responsabilité régionale $(R R \geq 3)$.

Ces catégories ne doivent pas être interprétées comme mesure directe ou indirecte de probabilité d'événement d'extinction et/ou de régression. Pour chacune des catégories ici définies [P1 à P4], nous proposons des actions d'amélioration des connaissances, lesquelles constituent les étapes nécessaires et préalables à toute action de conservation (Tableau 2).

\section{MESURE DU HANDICAP ET DES MOYENS DÉPLOYÉS}

\section{DANS LA CONSERVATION DES ESPÈCES}

En complément, nous proposons de mesurer les handicaps (note sur 10) et les moyens actuellement déployés (note sur 10) dans la conservation des espèces. Cette démarche vise à identifier des axes possibles d'actions à mener, afin d'améliorer les principaux champs de connaissances et/ou leur prise en compte dans les actions régionales de conservation (Tableaux 3, 4).

Le handicap à la conservation $(\mathrm{H})$ est évalué à travers le "handicap taxonomique» incluant les niveaux de difficulté de détermination des espèces (note sur 5) et le «handicap naturaliste" basé sur l'année de dernière observation sur la période 1977-2020 (note sur 5). Le niveau de détermination est utilisé comme proxy pour mesurer la proportion d'erreurs d'identification à travers un ensemble hétérogène d'observateurs et pour rendre compte de l'état des connaissances académiques sur les délimitations interspécifiques. L’année de dernière observation est ici utilisée comme mesure indirecte de la rareté des observations et donc de la probabilité de détecter une espèce soit à cause de sa rareté écologique soit de la difficulté d'accéder à ses (micro)habitats ou d'y récolter des spécimens.

Les moyens déployés $(\mathrm{M})$ dans la conservation des espèces sont mesurés à travers leur prise en compte par les textes réglementaires en faveur de la protection des espèces et de leurs habitats (note sur 5) et leur présence dans des espaces naturels réglementés (note sur 5). Pour le premier critère, les notes les plus hautes sont attribuées aux contraintes réglementaires les plus fortes. Pour le second critère, nous calculons le pourcentage d'occurrences par espèce situées à l'intérieur des périmètres des Réserves naturelles nationales et régionales (RNN, RNR), des Arrêtés préfectoraux de Protection de Biotope (APPB) et des Réserves biologiques dirigées et intégrales (RBD, RBI). Seules les observations ayant une précision géographique au point ou au lieu-dit sont utilisées.

Le détail de l'attribution des notes par critère ainsi que les actions compensatoires proposées sont donnés dans les Tableaux 3 et 4 .

Par ailleurs, le rapport $\mathrm{M} / \mathrm{H}$ indique pour des valeurs supérieures à 1 que les moyens déployés sont jugés favorables à la conservation des espèces et compensent $a$ priori les handicaps. Des actions de conservation peuvent être appliquées en fonction de la catégorisation des espèces cibles.

Si les moyens déployés ne permettent pas de compenser les handicaps, alors les actions d'amélioration des connaissances doivent être envisagées prioritairement $(\mathrm{M} / \mathrm{H}<1)$ ou concomitamment $(\mathrm{M} / \mathrm{H}=1)$ sur les actions de conservation.

L'ensemble des tests statistiques a été réalisé avec le logiciel R v3.6.3 (R Core Team 2020), la valeur seuil de significativité a été fixée ici à $5 \%$. La normalité et l'égalité des variances sont vérifiées systématiquement avant de réaliser un test paramétrique au moyen d'un test de ShapiroWilk pour la normalité et d'un test F pour l'égalité des variances. Dans le cas contraire, un test non paramétrique équivalent est réalisé. 
TABLEAU 3. - Critères pour la mesure du handicap à la conservation des espèces. Les actions compensatoires sont présentées par classe de note. Abréviation: Nsp, nombre d'espèces par classe de note de 1 à 5 .

\begin{tabular}{|c|c|c|c|c|c|c|}
\hline Note & $\begin{array}{l}\text { Handicap taxonomique } \\
\text { Niveaux de difficulté } \\
\text { pour l'identification } \\
\text { des espèces (d'après } \\
\text { Bichain et al. 2019) }\end{array}$ & Nsp & Actions compensatoires & $\begin{array}{l}\text { Handicap naturaliste } \\
\text { Année de dernière } \\
\text { observation (d'après } \\
\text { la base de données } \\
\text { globale) }\end{array}$ & Nsp & Actions compensatoires \\
\hline 5 & N5.1 & 29 & $\begin{array}{l}\text { A1. Favoriser les programmes } \\
\text { de recherche concernant } \\
\text { la délimitation/validité des } \\
\text { espèces }\end{array}$ & De 1977 à 1984 & 3 & $\begin{array}{l}\text { B1. Accentuer } \\
\text { significativement l'effort } \\
\text { d'échantillonnage à partir } \\
\text { de moyens adaptés }\end{array}$ \\
\hline 4 & N4 et N5.2 & 27 & $\begin{array}{l}\text { A2. Favoriser les démarches } \\
\text { adaptées d'identification } \\
\text { (barcoding, anatomie, } \\
\text { taxonomie intégrative, etc.) }\end{array}$ & De 1985 à 1994 & 7 & $\begin{array}{l}\text { (ADNe, cartographies } \\
\text { prédictives de } \\
\text { présence, programmes } \\
\text { transdisciplinaires ou }\end{array}$ \\
\hline 3 & N3+ & 46 & A3. Favoriser l'élaboration de & De 1995 à 2004 & 8 & transrégionaux/frontaliers, \\
\hline 2 & N3 & 66 & $\begin{array}{l}\text { supports didactiques et/ } \\
\text { ou l'aide à la formation pour } \\
\text { l'identification des espèces }\end{array}$ & De 2005 à 2014 & 10 & etc.) \\
\hline 1 & N1 à N2 & 59 & - & De 2015 à 2020 & 199 & - \\
\hline
\end{tabular}

TABLEAU 4. - Critères pour la mesure des moyens déployés dans la conservation des espèces. Les actions compensatoires sont présentées par classe de note. Abréviations: Bern, convention relative à la conservation de la vie sauvage et du milieu naturel de l'Europe; DHFF, Directive Habitats, Faune, Flore, Directive de l'Union européenne 92/43/CEE; Nsp, nombre d'espèces par classe de note de 1 à 5.

\begin{tabular}{|c|c|c|c|c|c|c|}
\hline Note & $\begin{array}{l}\text { Espèces réglementées } \\
\text { (textes) }\end{array}$ & Nsp & $\begin{array}{l}\text { Actions } \\
\text { compensatoires }\end{array}$ & $\begin{array}{l}\text { Présence dans un } \\
\text { espace réglementé } \\
\text { (d'après la base de } \\
\text { données globale) }\end{array}$ & Nsp & $\begin{array}{l}\text { Actions } \\
\text { compensatoires }\end{array}$ \\
\hline 5 & $\begin{array}{l}\text { Arrêté du } 23 \text { avril } 2007 \text { fixant les } \\
\text { listes des mollusques protégés } \\
\text { sur l'ensemble du territoire et } \\
\text { les modalités de leur protection, } \\
\text { article } 2 \text {; DHFF annexe(s) } 2 \text { et/ou } 4\end{array}$ & 3 & - & De $75 \%$ à $100 \%$ & 4 & - \\
\hline 4 & $\begin{array}{l}\text { Arrêté du } 23 \text { avril } 2007 \text { fixant les } \\
\text { listes des mollusques protégés } \\
\text { sur l'ensemble du territoire et les } \\
\text { modalités de leur protection, article } 3 \\
\text { ou } 4\end{array}$ & 9 & - & De $50 \%$ à $74 \%$ & 5 & - \\
\hline 3 & DHFF annexe 2 ou 4 & 4 & C1. Nécessité d'une & De $25 \%$ à $49 \%$ & 26 & D1. Favoriser la \\
\hline 2 & $\begin{array}{l}\text { Arrêté du } 24 \text { avril } 1979 \text { fixant la liste } \\
\text { des Escargots dont le ramassage et } \\
\text { la cession à titre gratuit ou onéreux } \\
\text { peuvent être interdits ou autorisés, } \\
\text { article1A ou 1B; } \\
\text { DHFF annexe 5; Bern annexe } 3\end{array}$ & 1 & $\begin{array}{l}\text { réglementation à } \\
\text { l'échelle nationale } \\
\text { et/ou régionale }\end{array}$ & De $5 \%$ à $24 \%$ & 112 & $\begin{array}{l}\text { réglementation } \\
\text { et/ou la gestion } \\
\text { conservatoire des } \\
\text { espaces de, ou } \\
\text { partie, de l'aire } \\
\text { d'occupation }\end{array}$ \\
\hline 1 & Aucun & 210 & & En dessous de $5 \%$ & 80 & \\
\hline
\end{tabular}

\section{RÉSULTATS}

\section{SÉLECTION DES DONNÉES D'OBSERVATION}

\section{ET D'OCCURRENCE}

La base de données initiale réunit un total de 85668 données $\left(\mathrm{N}_{\mathrm{obs}}\right)$, dont $47,7 \%$ ont été transmises par l'INPN. Le reste provient essentiellement des deux portails collaboratifs régionaux Faune Grand Est du réseau VisioNature et de la base TAXA du CBNFC-ORI (Conservatoire botanique national de Franche-Comté-Observatoire régional des Invertébrés). Le volume de données attribuables à un programme de science participative est estimé à environ 33700 données. Ce corpus d'informations est lié à 292 espèces de Mollusques continentaux actuels.
La première étape de traitement global (Fig. 1) permet d'éliminer environ 21000 données jugées ici inadéquates d'après les critères de précision taxonomique $\left(\mathrm{P}_{\operatorname{tax}}\right)$, géographique $\left(\mathrm{P}_{\text {geo }}\right)$, temporelle $\left(\mathrm{P}_{\text {temp }}\right)$ et d'identité d'observateur/collecteur $\left(\mathrm{P}_{\text {det }}\right)$. Le détail des différents taux de précision est donné sur les Figures 2A à 2D. Par ailleurs, nous écartons 20 taxons terminaux et leurs données afférentes, car considérés comme des introductions ponctuelles ou d'identifications douteuses (Annexe 1). Tous les taxons fossiles marins ou continentaux sont éliminés du jeu de données $\left(\mathrm{N}_{\mathrm{obs}}=205\right)$.

Le second traitement (Fig. 2E, F) permet d'éliminer environ 4000 données pour lesquelles la détermination est évaluée inadéquate sur le niveau d'expertise $\left(\mathrm{N}_{\mathrm{det}}\right)$. Enfin, l'élimination des données antérieures à 1977 permet d'écarter 


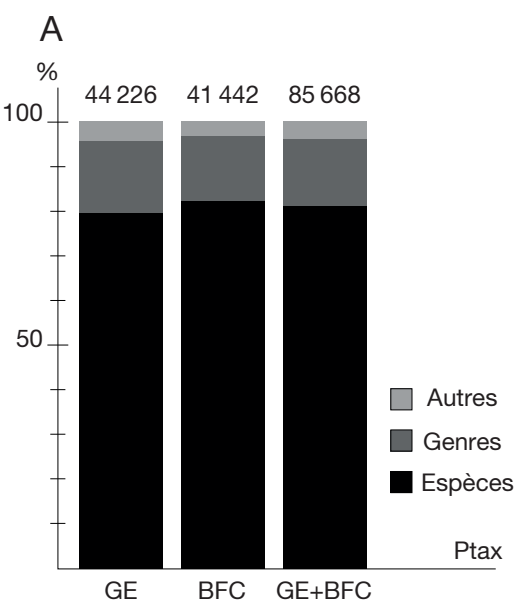

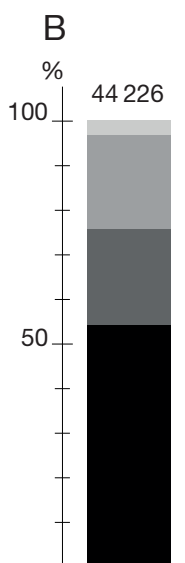

GE

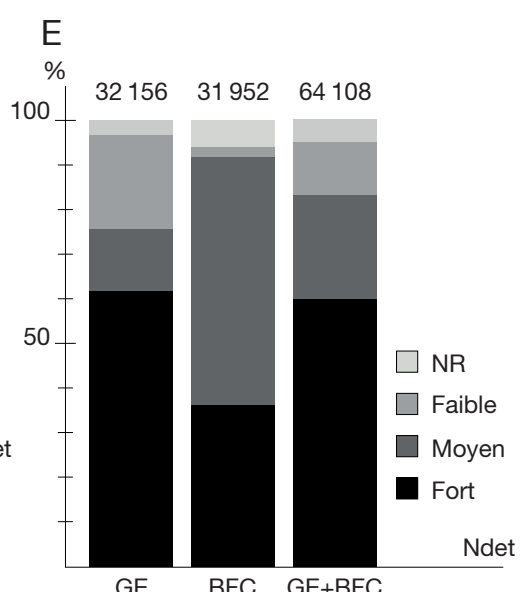

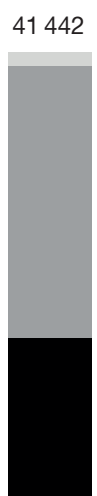

BFC

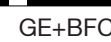

\section{8}

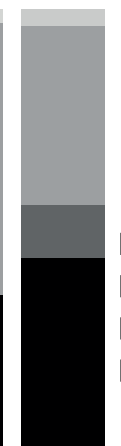

$\square$ Inadéquates

$\square$ Communes

$\square$ Toponymes

Coordonnées Pgeo

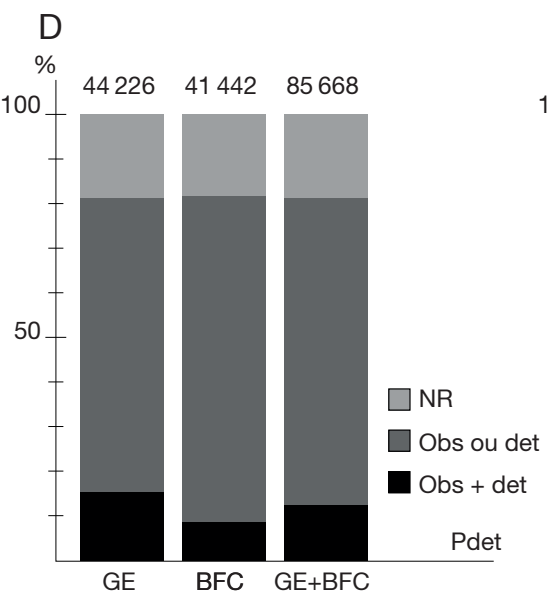

BFC GE+BFC

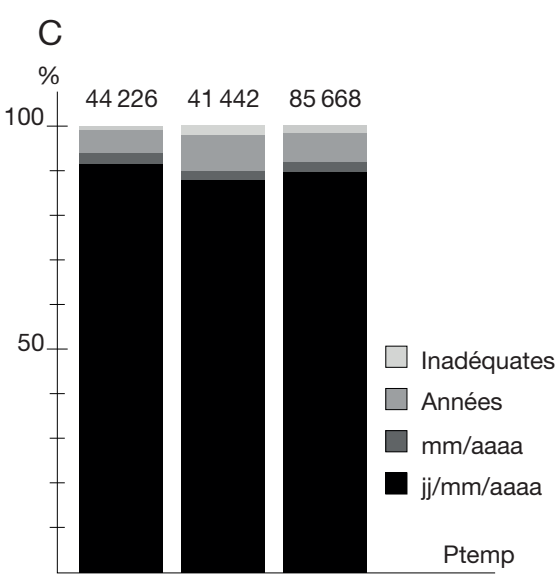

GE

BFC GE+BFC

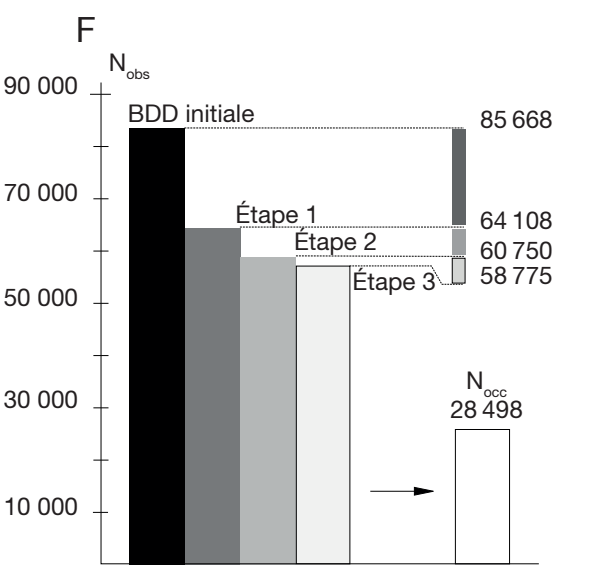

GE+BFC

G

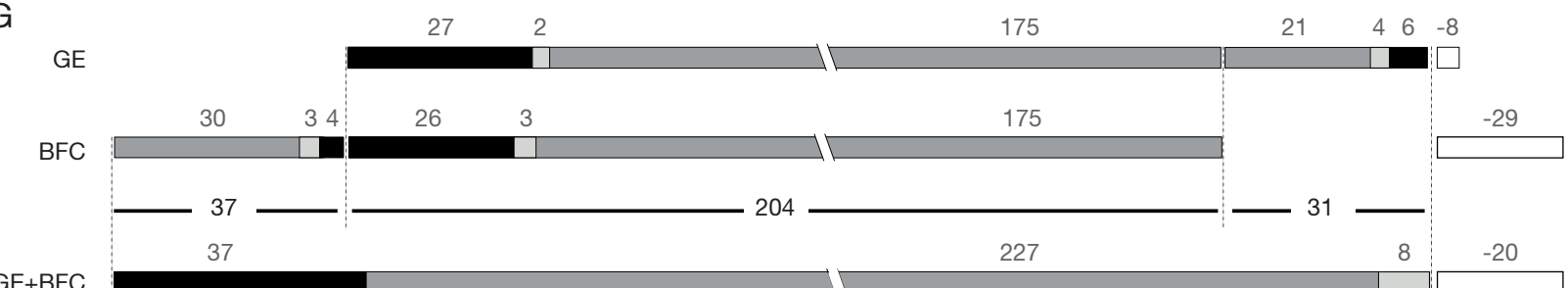

Espèces introduites

Espèces évaluées (données après 1977)

Espèces potentiellement éteintes (aucune données depuis 1977)

$\square$ Espèces exclues (déterminations douteuses)
GE Grand-Est - 235 espèces

BFC Bourgogne-Franche-Comté - 241

GE+BFC Aire d'étude - 272 espèces

FIG. 2. - Précision et répartition des informations dans la base de données initiale. A, Précisions taxonomiques $\mathbf{P}_{\text {tax }}$ au rang de l'espèce, du genre ou supragénérique pour autres; $\mathbf{B}$, précision géographique $\mathbf{P}_{\text {geo }}$ aux coordonnées, au toponyme à la commune ou région pour inadéquates; $\mathbf{C}$, précision temporelle $\mathbf{P}_{\text {temp }}$ au jour/mois/année, mois/année, année ou sans information de date pour inadéquates; $\mathbf{D}$, précision de la mention du déterminateur et/ou du récolteur $\mathbf{P}_{\text {det }} ; \mathbf{E}$, proportion des niveaux d'expertise $\mathbf{N}_{\text {det }} ; \mathbf{F}$, synthèse des volumes de données supprimés à chaque étape de traitement; $\mathbf{G}$, nombre d'espèces par grande subdivision administrative de l'aire d'étude. Abréviations; $\mathbf{N}_{\mathrm{obs}}$, données d'observation; $\mathbf{N}_{\mathrm{occ}}$, données d'occurrences; $\mathbf{N R}$, non renseigné.

environ 2000 données (Fig. 2F). Seuls huit taxons ne sont pas documentés depuis 1977. Ils sont conservés dans le jeu de données final et considérés ici comme potentiellement disparus.

Près de 31,4\% des données $\left(\mathrm{N}_{\mathrm{obs}}=26893\right)$ et $6,8 \%$ des espèces $\left(\mathrm{N}_{\mathrm{sp}}=20\right)$ sont écartés du corpus initial. Au total, 272 espèces sont liées à 28498 données d'occurrences $\left(\mathrm{N}_{\text {occ }}\right)$.
Parmi elles, 235 espèces appartiennent à la malacofaune de la région Grand Est et 241 à celle de Bourgogne-FrancheComté (Fig. 2G). Le taux de superposition entre les deux faunes régionales est de $75 \%$ soit 204 espèces en commun. Les différences entre la liste de référence du Grand Est (Bichain et al. 2019) et la présente liste ici retenue pour cette région sont données en Annexe 1. 


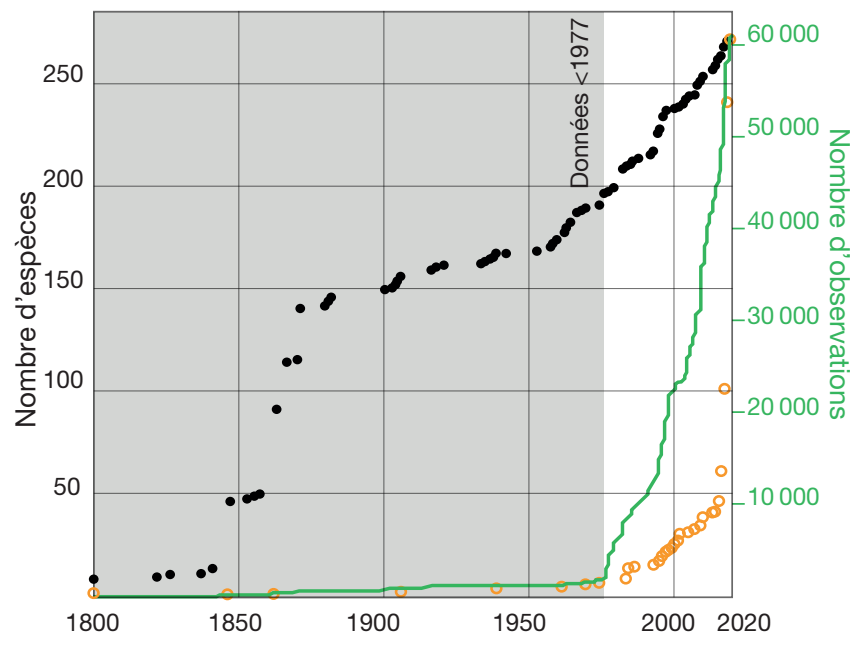

FIG. 3. - Courbe d'accumulation du nombre d'espèces et des données d'observation. Les points noirs indiquent le nombre cumulé d'espèces en fonction de la première mention d'observation, les points orange indiquent le nombre cumulé d'espèces en fonction de la dernière mention d'observation. En vert, le nombre cumulé de données d'observations $\mathrm{N}_{\mathrm{obs}}$. La zone grisée indique les données d'observation antérieures à 1977 qui ne sont pas prises en compte dans le jeu de données finale. Les huit espèces non revues depuis 1977 sont considérées comme potentiellement éteintes.

La courbe d'accumulation du nombre d'observations $\mathrm{N}_{\text {obs }}$ par année (Fig. 3) montre que plus de $95 \%$ du volume total de données a été acquis depuis 1977 et près de $50 \%$ sur les 10 dernières années. Sur les 272 taxons enregistrés, $97 \%$ ont été documentés depuis 1977 et $85 \%$ sur les cinq dernières années. L'écart moyen entre la première et dernière année d'observation est de 101,10 années $(\mathrm{EC}=69,69)$.

\section{PARTITIONS TAXONOMIQUES}

Parmi l'ensemble des espèces de l'étude, 234 appartiennent à la classe des Gastéropodes et 38 à celle des Bivalves (Fig. 4A). Les milieux aquatiques comptent 105 espèces (38 Bivalves et 67 Gastéropodes) vs 167 espèces pour les milieux terrestres. Globalement, 37 espèces (13,6\%) sont des éléments exogènes (introduites dont certaines sont des introduites envahissantes) à la faune régionale ou supposés comme tels (cryptogènes), alors que le taux d'endémisme parmi les taxons indigènes est d'environ 11,5\% (Fig. 4B). Quant aux niveaux de difficulté de détermination (Fig. 4D), $29 \%$ des taxons sont aisément déterminables par les malacologues amateurs (catégories $\mathrm{N} 1$ et N2), $26 \%$ nécessitent des compétences renforcées (N3), $33 \%$ une approche d'expertise (N3+, N4 et N5.2) et $12 \%$ ont des délimitations spécifiques controversées (N5.1). Par ailleurs, $94,19 \%$ des espèces $\left(\mathrm{N}_{\mathrm{sp}}=162\right)$ sont identifiées par des experts et $11,76 \%\left(\mathrm{~N}_{\mathrm{sp}}=32\right)$ à travers les protocoles $\mathrm{ADNe}($ Fig. $4 \mathrm{C})$ avec $60 \%$ de ces taxons catégorisés de N3 à N5 (Fig. 4E).

Cependant, cette répartition d'ensemble des taux d'indigénat, d'endémisme, de niveaux de difficulté de détermination ou d'expertise n'est pas homogène à travers les différentes partitions (Fig. 4A). En effet, le groupe informel des Méso-Bivalves (Corbicula spp., Dreissena spp.) compte quatre espèces, toutes considérées comme introduites envahissantes et faciles de détermination, lesquelles sont majoritairement documentées par des données d'expertise faible ou moyenne. En revanche, le groupe informel des Hydrobies concentre le plus fort taux d'endémisme $\left(\mathrm{N}_{\mathrm{sp}}=22 ; 81,5 \%\right)$ ainsi que la plus haute part en taxons dont la validité taxonomique est sujette à discussion $\left(\mathrm{N}_{\mathrm{sp}}=23 ; 85,2 \%\right)$. Les groupes des Limaces et des Micro-Bivalves concentrent par ailleurs la plus forte part d'espèces nécessitant des approches d'expertise (N3+, N4 et $\mathrm{N} 5.2)$, respectivement $65 \%\left(\mathrm{~N}_{\mathrm{sp}}=15\right)$ et $91 \%\left(\mathrm{~N}_{\mathrm{sp}}=21\right)$ à l'inverse du groupe des Macro-Bivalves $\left(\mathrm{N}_{\mathrm{sp}}=1 ; 10 \%\right)$. D’une manière générale, le volume de données expertes reste majoritaire dans chaque partition à l'exception des Macro- et Méso-Bivalves.

\section{ÉVALUATION DES BIAIS D’OCCURRENCES Biais géographiques}

Les résultats sont fournis dans la Figure 5 et le Tableau 5. Sur l'ensemble de l'aire d'étude, environ $12 \%$ des mailles de $2 \times 2 \mathrm{~km}\left(\mathrm{~N}_{\mathrm{m}}=3462\right)$ ont au moins une donnée d'observation alors que le nombre total d'observations $\left(\mathrm{N}_{\mathrm{obs}}=60726\right)$ rapporté par unité de surface est de $0,57 \mathrm{obs} / \mathrm{km}^{2}\left(\mathrm{~N}_{\mathrm{obs}} / \mathrm{N}_{\mathrm{t}}\right)$. En moyenne, chaque maille échantillonnée est documentée par 17,54 données d'observation $\left(\mathrm{N}_{\mathrm{obs}} / \mathrm{N}_{\mathrm{m}}\right)$ et le volume de données expertes $\left(\mathrm{N}_{\text {exp }}\right)$ représente $64 \%$ des observations.

Cependant, les valeurs pour ces quatre paramètres montrent de fortes disparités à travers les différentes subdivisions administratives, sans corrélation significative avec leur surface notamment (test de corrélation de Person ou de Spearman, p >>0,05). Globalement, les territoires ne sont ni échantillonnés de manière proportionnelle à leur surface, ni de manière homogène d'un point de vue d'effort global d'échantillonnages. Les différences sont atténuées à l'échelle de comparaison des deux régions Grand Est et Bourgogne-Franche-Comté, dont les surfaces sont de même grandeur.

L'effort global d'observation est considéré comme satisfaisant dans les départements du Doubs, de la Haute-Saône, du Bas-Rhin, du Jura et de la Meuse et très faible dans les départements de la Haute-Marne, de la Nièvre, des Vosges et de l'Yonne (Fig. 5). Pour ces derniers, il faudrait doubler voire tripler l'effort d'observation actuel pour obtenir des niveaux comparables avec les territoires les mieux étudiés.

Lécart entre l'estimation du nombre d'espèces par Chao 2 et le nombre d'espèces documentées par département présente une corrélation inverse avec la mesure de l'effort d'échantillonnage (test de corrélation de Spearman, $\mathrm{p}=0,06617$, rho 0,44), proche du seuil de significativité indiquant une influence forte entre la qualité de l'estimation du nombre d'espèces par Chao2 et notre estimateur global d'effort d'échantillonnage.

Par ailleurs, les catégories de départements qui présentent les efforts d'observation les plus faibles ont un nombre élevé de singletons (= observation unique) (Tableau 5: F1, L), qui en moyenne sont significativement plus élevés que sur l'ensemble des départements (pour F1: moyenne $=28,75 ; \mathrm{EC}=9,10$ vs moyenne $=20,44 ; \mathrm{EC}=7,64$ et pour $\mathrm{L}:$ moyenne $=34,5$; $\mathrm{EC}=9,45$ vs moyenne $=25,83 ; \mathrm{EC}=8,43)$. Il existe une corrélation inverse et significative entre les valeurs de F1 et de $\mathrm{L}$ avec la mesure de l'effort d'observation (tests de corrélation de Pearson, $\mathrm{p}=5 \times 10^{-4}$; $\left.\operatorname{cor} \sim 0,74\right)$. 
Macro-bivalves

\begin{tabular}{|lr|lr|lr|}
\hline \multicolumn{2}{|l|}{ Statut } & Niv. tax. & $\% \mathrm{~N}_{\text {det. }}$ \\
\hline Int & 1 & $\mathrm{~N} 1$ & 0 & $\mathrm{NR}$ & 20 \\
End & 0 & $\mathrm{~N} 2$ & 10 & $\mathrm{Nf}$ & 47 \\
EndR & 0 & $\mathrm{~N} 3$ & 0 & & $\mathrm{~N} 3+$ \\
SEnd & 0 & 0 & $\mathrm{Nm}$ & 6 \\
PoDi & 1 & $\mathrm{~N} 5.1$ & 0 & $\mathrm{Ne}$ & 25 \\
Ind & 9 & $\mathrm{~N} 5.2$ & 1 & Nadn 3 \\
\hline
\end{tabular}

GE : 11 / BFC : 11 / GE+BFC : 11

Limaces

\begin{tabular}{|lr|lr|ll|}
\hline Statut & \multicolumn{2}{|l|}{ Niv. tax. } & \% \\
Int & \\
End & 9 & N1 & 5 & NR & 2 \\
EndR & 0 & N3 & 3 & Nf & 15 \\
SEnd & 0 & N3+ & 1 & Nm & 25 \\
PoDi & 1 & N5.1 & 13 & Ne r & 58 \\
Ind & 13 & N5.2 & 0 & Nadn & 0 \\
\hline
\end{tabular}

GE : 23 / BFC : 18 / GE+BFC : 23

Escargots

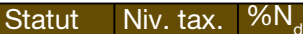

\begin{tabular}{|lr|lr|ll|}
\hline Int & 15 & $\mathrm{~N} 1$ & 18 & $\mathrm{NR}$ & 1 \\
End & 2 & $\mathrm{~N} 2$ & 18 & $\mathrm{Nf}$ & 8 \\
EndR & 0 & $\mathrm{~N} 3$ & 59 & & $\mathrm{~N} 3+$ \\
SEnd & 1 & $\mathrm{~N} 4$ & 14 & $\mathrm{Nm}$ & 36 \\
PoDi & 4 & $\mathrm{~N} 5.1$ & 8 & $\mathrm{Ne}$ & 54 \\
Ind & 122 & $\mathrm{~N} 5.2$ & 3 & Nadn & 0 \\
\hline
\end{tabular}

GE : 127 / BFC : 128 / GE+BFC : 144

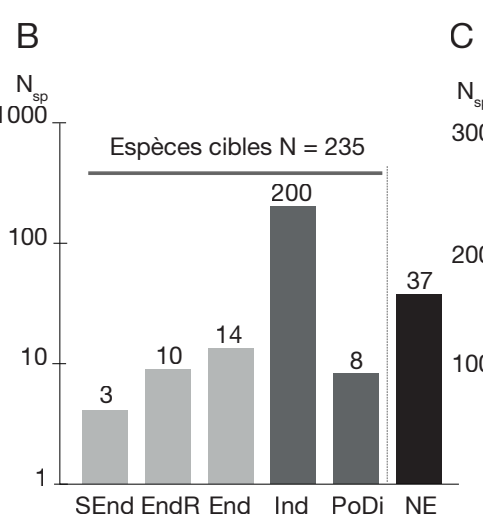

C

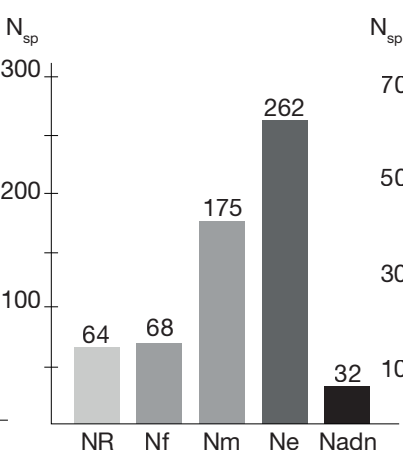

Méso-bivalves

\begin{tabular}{|ll|ll|lr|}
\hline Statut & \multicolumn{2}{|l|}{ Niv. tax. } & $\% \mathrm{~N}_{\text {det. }}$ \\
\hline Int & 4 & $\mathrm{~N} 1$ & 0 & $\mathrm{NR}$ & 15 \\
End & 0 & $\mathrm{~N} 2$ & 4 & $\mathrm{Nf}$ & 37 \\
EndR & 0 & $\mathrm{~N} 3$ & 0 & $\mathrm{Nm}$ & 6 \\
SEnd & 0 & $\mathrm{~N} 3+$ & 0 & $\mathrm{Nm}$ & 0 \\
PoDi & 0 & $\mathrm{~N} 5.1$ & 0 & $\mathrm{Ne}$ & 39 \\
Ind & 0 & $\mathrm{~N} 5.2$ & 0 & $\mathrm{Nadn}$ & 4 \\
\hline
\end{tabular}

GE : 4 / BFC : 4 / GE+BFC : 4

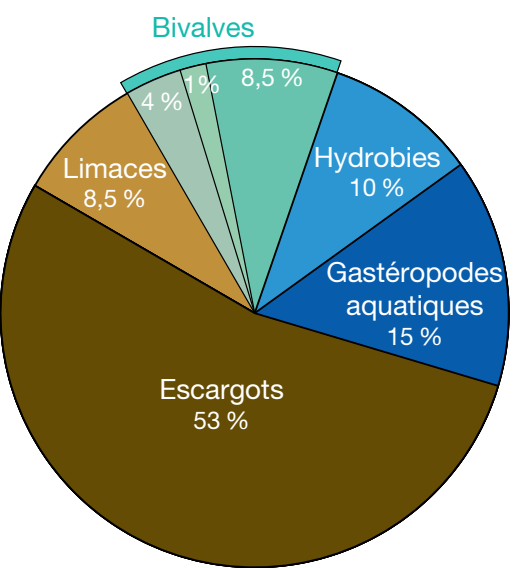

Micro-bivalves

\begin{tabular}{|c|c|c|}
\hline Statut & Niv. tax. & $\% \mathrm{~N}_{\text {det. }}$. \\
\hline & N1 & NR \\
\hline End & N2 & $\mathrm{Nf}$ \\
\hline EndR 0 & N3 & 101 \\
\hline SEnd 0 & $\begin{array}{ll}\text { N3+ } & 17 \\
\text { N4 }\end{array}$ & $\mathrm{Nm}$ \\
\hline PoDi 0 & N5.1 & $\mathrm{Ne} \quad 95$ \\
\hline Ind 22 & N5.2 & Nadn 4 \\
\hline
\end{tabular}

Hydrobies

\begin{tabular}{|c|c|c|}
\hline Statut & Niv. tax. & $\% \mathrm{~N}_{\text {det. }}$ \\
\hline Int & N1 & NR \\
\hline End 12 & N2 & $\mathrm{Nf}$ \\
\hline EndR 10 & N3 & \\
\hline SEnd 2 & N3+ & $\mathrm{Nm}$ \\
\hline PoDi 2 & N5.1 23 & $\mathrm{Ne} \quad 90$ \\
\hline Ind & N5.2 & Nadn 0 \\
\hline
\end{tabular}

Gastéropodes aquatiques

\begin{tabular}{|c|c|c|c|}
\hline Statut & Niv. ta & & $\% \mathrm{~N}_{\text {det. }}$ \\
\hline Int & N1 & 3 & NR 2 \\
\hline End & N2 & 15 & $\mathrm{Nf}$ \\
\hline EndR & N3 & 6 & IVI \\
\hline SEnd & N3+ & 10 & $\mathrm{Nm} \quad 7$ \\
\hline PoDi & $\begin{array}{l}\text { IN4 } \\
\text { N5.1 }\end{array}$ & $\begin{array}{l}4 \\
0\end{array}$ & $\mathrm{Ne} \quad 88$ \\
\hline Ind & N5.2 & 2 & Nadn 0 \\
\hline
\end{tabular}

E

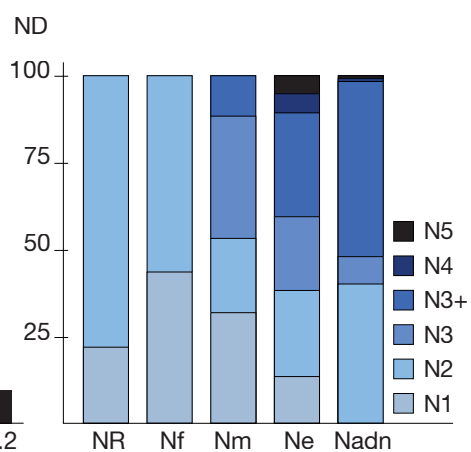

FIG. 4. - Partitions taxonomiques. A, Pourcentage d'espèces par partition taxonomique et pour chaque partition, le nombre espèces endémique (End), micro-endémique (EndR), indigène (Ind), introduite (Int), potentiellement éteintes (PoDi) ou subendémique (SEnd); par niveau de difficulté de détermination d'après Bichain et al. (2019); par niveau d'expertise des observateurs en pourcentage non renseigné (NR), faible (Nf), moyen (Nm), expert (Ne), protocole ADNe (Nadn); B, répartition du nombre d'espèces sur l'ensemble du jeu de données final par statut d'indigénat; $\mathbf{C}$, répartition sur l'ensemble du jeu de données final du nombre d'espèces par niveau d'expertise des observateurs; $\mathbf{D}$, répartition sur l'ensemble du jeu de données final du nombre d'espèces par niveau de difficulté de détermination; E, répartition sur l'ensemble du jeu de données final du nombre d'espèces par niveau de difficulté de détermination pour chaque classe de niveau d'expertise. Les taxons classés dans N1 à N3 peuvent être identifiés sur la base de coquilles seules; pour N3+ à N4, l'anatomie est nécessaire ou indispensable pour une identification fiable; pour N5.1 et N5.2, l'apport de données moléculaires peut être nécessaire pour une identification fiable. La délimitation spécifique des taxons classés dans N5.1 est sujette à débat.

En d'autres termes, le nombre de données uniques est d'autant plus élevé qu'un département est considéré comme présentant un déficit global d'informations. Cela indique une proportion élevée de données opportunistes, lesquelles sont moins informatives que les observations issues d'inventaires spécifiques ou de travaux de recherches. Ces résultats permettent donc d'identifier les territoires où l'inventaire taxonomique ne reflète pas la réelle richesse spécifique. Les estimations du nombre d'espèces pour ces différentes subdivisions géographiques sont données dans le Tableau 5.

\section{Biais taxonomiques}

L'application de la méthode de Troudet et al. (2017) montre que les différentes partitions taxonomiques ne sont pas étudiées proportionnellement à leur nombre d'espèces. D'une manière générale, les Gastéropodes aquatiques, les Limaces et les Hydrobies présentent des déficits globaux d'observations à l'échelle de la zone d'étude (Fig. 6). Le constat est identique entre les partitions, pour lesquelles le nombre d'espèces et les macro-habitats sont comparables, comme les Micro-Bivalves $\left(\mathrm{N}_{\mathrm{occ}}=+316 ; \mathrm{N}_{\mathrm{sp}}=23\right)$ vs les Hydrobies $\left(\mathrm{N}_{\mathrm{occ}}=-2425\right.$; 


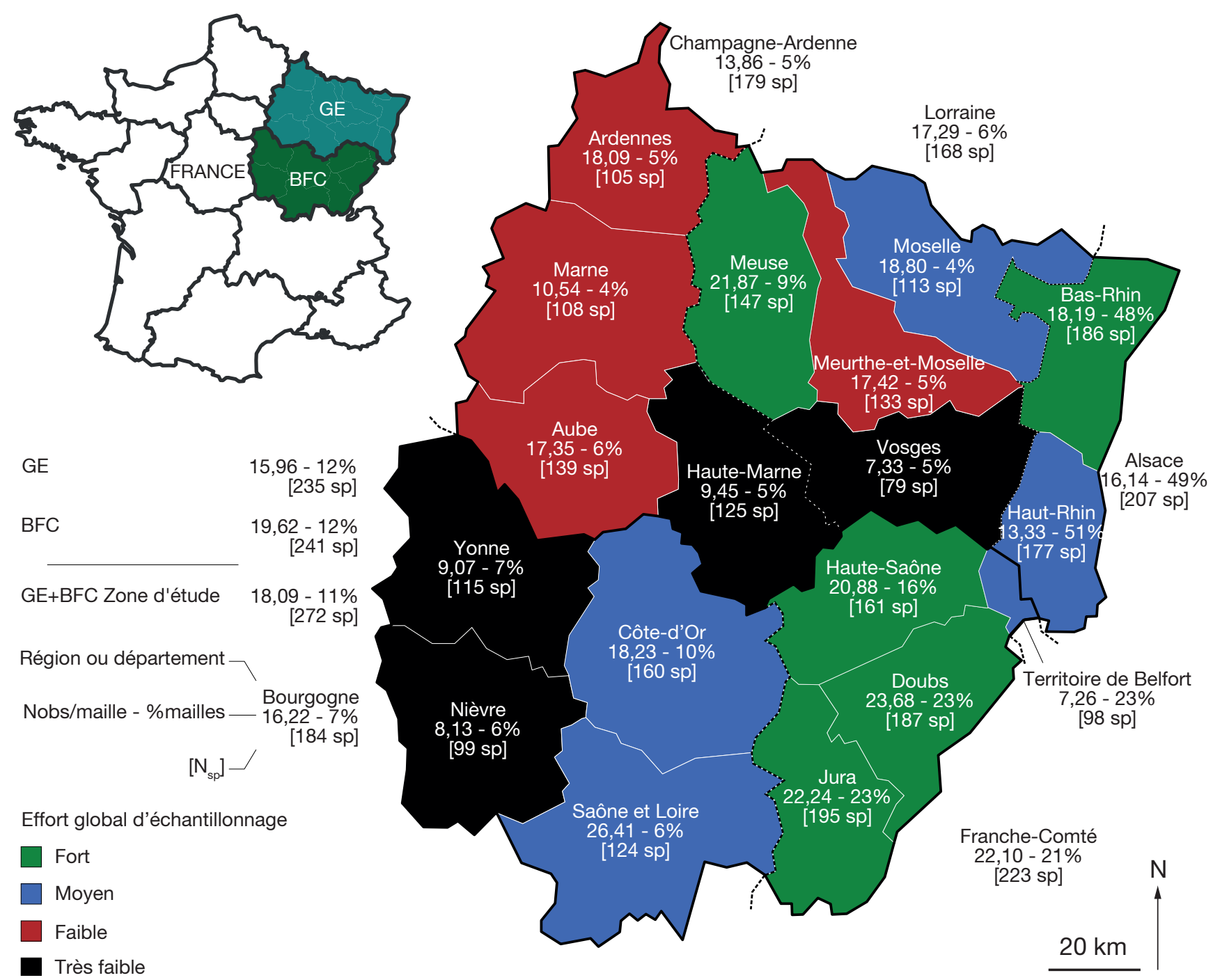

FIG. 5. - Biais géographiques dans les données d'observation et d'occurrences. Le code couleur indique les catégories d'effort global d'échantillonnage (Tableau 4). Abréviations: \%mailles, nombre de mailles documentées par au moins une donnée d'observation rapporté au nombre total de mailles; BFC, Bourgogne-Franche-Comté; GE, Grand-Est; Nobs/maille, nombre moyen d'observations par maille; $\mathbf{N}_{\text {sp }}$, nombre d'espèces d'après le jeu de données global.

$\left.\mathrm{N}_{\mathrm{sp}}=27\right)$ ou pour les Bivalves s.l. $\left(\mathrm{N}_{\mathrm{occ}}=+697 ; \mathrm{N}_{\mathrm{sp}}=38\right)$ vs Gastéropodes aquatiques $\left(\mathrm{N}_{\mathrm{occ}}=-506 ; \mathrm{N}_{\mathrm{sp}}=40\right)$.

La même méthode a été appliquée, mais en regroupant les espèces selon les cinq classes de handicap taxonomique ou de réglementation des espèces (Tableau 3 ). Nous montrons que les classes d'espèces catégorisées en N1 et N2, c'est-à-dire aisément identifiables par les malacologues amateurs, sont globalement surreprésentées $\left(\mathrm{N}_{\mathrm{occ}}=+5607 ; \mathrm{N}_{\mathrm{sp}}=79\right)$, ainsi que les classes d'espèces ayant soit un statut de protection nationale peu contraignante $\left(\mathrm{N}_{\mathrm{occ}}=+877 ; \mathrm{N}_{\mathrm{sp}}=3\right)$, soit un double statut de protection nationale et européenne $\left(\mathrm{N}_{\mathrm{occ}}=+131 \mathrm{~N}_{\mathrm{sp}}=4\right)$. C'est particulièrement le cas pour Unio crassus Philipsson, 1788 qui comptabilise près d'un tiers du nombre total d'occurrences des Macro-Bivalves. Les espèces qui ne bénéficient d'aucun statut de protection affichent une balance déficit/excès relativement neutre $\left(\mathrm{N}_{\mathrm{occ}}=+74 ; \mathrm{N}_{\mathrm{sp}}=252\right)$.

À l'inverse, les classes d'espèces catégorisées de N3 à N5.2, c'est-à-dire difficilement identifiables par les malacologues amateurs (N3 à N4 et N5.2) ou dont les descripteurs spécifiques sont discutés (N5.1), sont largement sous-représentées $\left(\mathrm{N}_{\mathrm{occ}}=-5114 ; \mathrm{N}_{\mathrm{sp}}=63\right.$ uniquement pour les niveaux $\mathrm{N} 4 \mathrm{et}$ $\mathrm{N} 5.1$ ), ainsi que la classe d'espèces ayant un unique statut de protection nationale $\left(\mathrm{N}_{\mathrm{occ}}=-873 ; \mathrm{N}_{\mathrm{sp}}=9\right)$ ou européenne $\left(\mathrm{N}_{\text {occ }}=-209 ; \mathrm{N}_{\mathrm{sp}}=4\right)$.

L'origine de ces biais reste difficilement identifiable. Nos résultats indiquent que les partitions taxonomiques surreprésentées ont, en proportion, majoritairement des taxons aisément identifiables par le plus grand nombre d'observateurs et/ou bénéficiant de fortes contraintes réglementaires notamment pour les Macro-Bivalves. À l'inverse, les partitions taxonomiques sous-représentées ont majoritairement des taxons soit avec un niveau d'identification spécifique élevé (Limaces), soit avec des délimitations spécifiques ambigües (Hydrobies). Pour les Limaces, les déficits sont aussi liés à la manière dont les observations sont réalisées. En effet, ces animaux ne laissent pas de coquilles vides identifiables au 
TABLEAU 5. - Effort d'échantillonnage par départements. Abréviations: Cat, note finale obtenue pour la catégorisation de l'effort d'échantillonnage, le score le plus haut représente l'effort d'échantillonnage le plus faible, en vert, effort d'échantillonnage fort, en bleu moyen, en rouge, faible, en noir, très faible; Chao2, nombre théorique d'espèces d'après l'algorithme Chao2; ChaoEC, écart-type associé à l'estimateur Chao2; Dpt, département; EC, écart type associé à $\mathrm{N}_{\text {obs }} / \mathrm{N}_{\text {maille }}$; $\mathbf{F 1}$, nombre d'espèce citée dans une seule maille; $\mathbf{L}$, nombre d'espèces avec une observation unique; $\mathbf{N}_{\text {exp }}$, proportion de données expertes; $\mathbf{N}_{\text {maille }}$, nombre de mailles $2 \times 2 \mathrm{~km}$ documentées par au moins une donnée d'observation; $\mathbf{N}_{\mathrm{mt}}$, nombre total de mailles $2 \times 2 \mathrm{~km} ; \mathbf{N}_{\text {obs }}$, Nombre d'observation; $\mathbf{N}_{\text {obs }} / \mathbf{N}_{\text {maille, }}$, nombre moyen d'observations par maille documentée; $\mathbf{N}_{\mathbf{s p}}$, nombre total d'espèces; $\mathbf{S t}$, Surface en km². Quartiles pour la catégorisation des classes d'effort d'échantillonnage (4 points sont attribués pour l'intervalle Q1-Q2, 3 points pour l'intervalle Q2-Q3, 2 points pour l'intervalle Q3-Q4 et 1 point pour l'intervalle

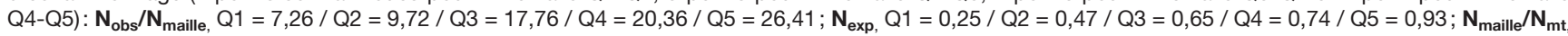
$\mathrm{Q} 1=0,04 / \mathrm{Q} 2=0,05 / \mathrm{Q} 3=0,06 / \mathrm{Q} 4=0,21 / \mathrm{Q} 5=0,51 ; \mathbf{N}_{\text {obs }} / \mathrm{St}, \mathrm{Q} 1=0,10 / \mathrm{Q} 2=0,18 / \mathrm{Q} 3=0,36 / \mathrm{Q} 4=0,83 / \mathrm{Q} 5=2,48$.

\begin{tabular}{|c|c|c|c|c|c|c|c|c|c|c|c|c|c|c|c|}
\hline \multirow[b]{2}{*}{ Dpt } & \multirow[b]{2}{*}{ Cat } & \multicolumn{5}{|c|}{ Variables descriptives } & \multicolumn{5}{|c|}{ Variables impliquées dans Cat } & \multicolumn{4}{|c|}{ Estimation } \\
\hline & & $\mathbf{N}_{\mathrm{sp}}$ & $\mathbf{N}_{\text {maille }}$ & $\mathbf{N}_{\mathbf{m t}}$ & $\mathbf{N}_{\text {obs }}$ & St & $\mathbf{N}_{\text {obs }} / \mathbf{N}_{\text {maille }}$ & EC & $\mathbf{N}_{\exp }$ & $\mathbf{N}_{\text {maille }} / \mathbf{N}_{\text {mt }}$ & $\mathbf{N}_{\text {obs }} / \mathrm{St}$ & Chao2 & ChaoEC & $\mathbf{L}$ & F1 \\
\hline Doubs & 5 & 187 & 335 & 1462 & 7932 & 5266 & 23,68 & 39,88 & 0,67 & 0,23 & 1,51 & 205,94 & 11,26 & 20 & 14 \\
\hline Jura & 5 & 195 & 323 & 1404 & 7182 & 5049 & 22,24 & 41,82 & 0,73 & 0,23 & 1,42 & 221,17 & 15,43 & 21 & 20 \\
\hline $\begin{array}{l}\text { Haute- } \\
\text { Saône }\end{array}$ & 5 & 161 & 239 & 1483 & 4991 & 5390 & 20,88 & 72,55 & 0,93 & 0,16 & 0,93 & 168,52 & 4,97 & 17 & 13 \\
\hline Meuse & 6 & 147 & 156 & 1714 & 3412 & 6211 & 21,87 & 34,29 & 0,92 & 0,09 & 0,55 & 153,07 & 4,95 & 11 & 9 \\
\hline Bas-Rhin & 6 & 187 & 649 & 1351 & 11804 & 4755 & 18,19 & 30,29 & 0,66 & 0,48 & 2,48 & 200,13 & 7,98 & 19 & 13 \\
\hline Haut-Rhin & 8 & 177 & 502 & 982 & 6691 & 3525 & 13,33 & 20,66 & 0,51 & 0,51 & 1,90 & 191,97 & 8,64 & 21 & 15 \\
\hline Moselle & 10 & 113 & 71 & 1756 & 1335 & 6216 & 18,80 & 49,71 & 0,89 & 0,04 & 0,21 & 121,13 & 4,8 & 22 & 14 \\
\hline $\begin{array}{l}\text { Territoire } \\
\text { de Belfort }\end{array}$ & 10 & 98 & 46 & 204 & 334 & 611 & 7,26 & 12,28 & 0,65 & 0,23 & 0,55 & 116,45 & 8,53 & 33 & 23 \\
\hline Côte-d'Or & 10 & 160 & 231 & 2366 & 4211 & 8802 & 18,23 & 43,44 & 0,28 & 0,10 & 0,48 & 186,88 & 13,49 & 28 & 19 \\
\hline $\begin{array}{l}\text { Saône-et- } \\
\text { Loire }\end{array}$ & 10 & 124 & 143 & 2350 & 3776 & 8614 & 26,41 & 64,54 & 0,36 & 0,06 & 0,44 & 145,08 & 11,4 & 24 & 22 \\
\hline Aube & 11 & 139 & 101 & 1644 & 1752 & 6139 & 17,35 & 26,87 & 0,72 & 0,06 & 0,29 & 159,93 & 9,92 & 31 & 24 \\
\hline Marne & 12 & 108 & 80 & 2223 & 843 & 8169 & 10,54 & 19,10 & 0,84 & 0,04 & 0,10 & 132,49 & 11,42 & 32 & 27 \\
\hline $\begin{array}{l}\text { Meurthe-et- } \\
\text { Moselle }\end{array}$ & 12 & 133 & 84 & 1531 & 1463 & 5246 & 17,42 & 28,87 & 0,74 & 0,05 & 0,28 & 147,71 & 8,12 & 23 & 16 \\
\hline Ardennes & 13 & 105 & 74 & 1453 & 1339 & 5229 & 18,09 & 16,17 & 0,39 & 0,05 & 0,26 & 119,8 & 7,86 & 25 & 24 \\
\hline $\begin{array}{l}\text { Haute- } \\
\text { Marne }\end{array}$ & 15 & 125 & 94 & 1718 & 888 & 6211 & 9,45 & 11,92 & 0,60 & 0,05 & 0,14 & 171,89 & 19,26 & 44 & 36 \\
\hline Vosges & 15 & 79 & 87 & 1639 & 638 & 5874 & 7,33 & 10,15 & 0,57 & 0,05 & 0,11 & 104,83 & 12,87 & 28 & 19 \\
\hline Yonne & 15 & 115 & 135 & 2028 & 1224 & 7462 & 9,07 & 15,12 & 0,46 & 0,07 & 0,16 & 130,22 & 8,23 & 24 & 23 \\
\hline Nièvre & 16 & 99 & 112 & 1871 & 911 & 6875 & 8,13 & 20,35 & 0,25 & 0,06 & 0,13 & 157,28 & 24,66 & 42 & 37 \\
\hline
\end{tabular}

rang spécifique, ont une activité principalement nocturne et, conséquemment, passent aisément inaperçues. En ce qui concerne les Hydrobies, les déficits ont également pour origine des aires de répartition réduites liées au micro-endémisme et/ou à la difficulté d'accéder à leur habitat notamment pour les espèces stygobies. Leur petite taille, souvent inférieure à $2 \mathrm{~mm}$, est probablement un handicap supplémentaire pour l'observation de ces animaux.

\section{LISTE HIÉRARCHISÉE DES ESPÈCES}

\section{À ENJEUX DE CONSERVATION}

La procédure de hiérarchisation a été appliquée sur 227 espèces cibles dont 202 sont présentes dans le Grand Est et 210 en Bourgogne-Franche-Comté. Aucune espèce n'obtient la note maximale théorique de 125 et les scores globaux s'étalent entre 1 et 80. La méthode de classification par ordre décroissant des scores globaux, puis des notes obtenues respectivement sur les critères $R R, V$ et $R$ permet de définir 46 rangs de priorité d'enjeux de conservation (Fig. 7). La classification obtenue et le détail des notes par critère sont donnés en Annexe 2 en suivant une présentation par partition taxonomique.

Concernant les catégories de risque, seulement $6 \%$ des espèces sont placées en $\mathrm{P} 1$ où les risques d'extinction globale sont les plus élevés et $9 \%$ en P2 laquelle est associée à un risque élevé d'extinctions globales et/ou locales. La catégorie $\mathrm{P} 3$, pour laquelle les risques d'extinction locale sont plus ou moins élevés, comptabilise près de $35 \%$ des taxons.
D’une manière générale, les critères de rareté $(R)$ et de responsabilité régionale $(\mathrm{RR})$ sont déterminants dans leur participation au score global notamment pour les espèces catégorisées en $\mathrm{P} 1$ avec respectivement 23 et 12 espèces ayant obtenus la note maximale. En P2, les trois critères participent de manière plus homogène avec cependant une participation plus forte de R, tendance que l'on observe aussi en P3.

La répartition des catégories de risque par partition taxonomique est donnée sur la Figure 7. Les Hydrobies et les MacroBivalves apparaissent comme les groupes avec les plus grandes proportions en espèces catégorisées en $\mathrm{P} 1$ et P2. De la même manière, les enjeux de conservation semblent se concentrer sur les taxons des milieux aquatiques.

Nous avons traité à part les espèces considérées comme potentiellement disparues. En effet, la pauvreté des données sur leur répartition ancienne ne permet pas de statuer ni sur leur réelle présence dans la région dans le passé, ni d'une éventuelle extinction plus ou moins récente. Les actions liées à cette catégorie sont par conséquent des recherches extensives et ciblées de ces taxons dans les habitats qui leur sont favorables. Nous considérons ces actions comme prioritaires.

\section{ACTIONS COMPENSATOIRES AU DÉFICIT DE CONNAISSANCES} On observe des différences significatives entre les valeurs moyennes de $(\mathrm{M} / \mathrm{H})$ des différentes catégories de risque P1 à $\mathrm{P} 4$ (test de Kruskal-Wallis, p-value $=0,001$ ). Les moyennes de $(\mathrm{M} / \mathrm{H})$ des catégories de risque les plus élevés sont signi- 


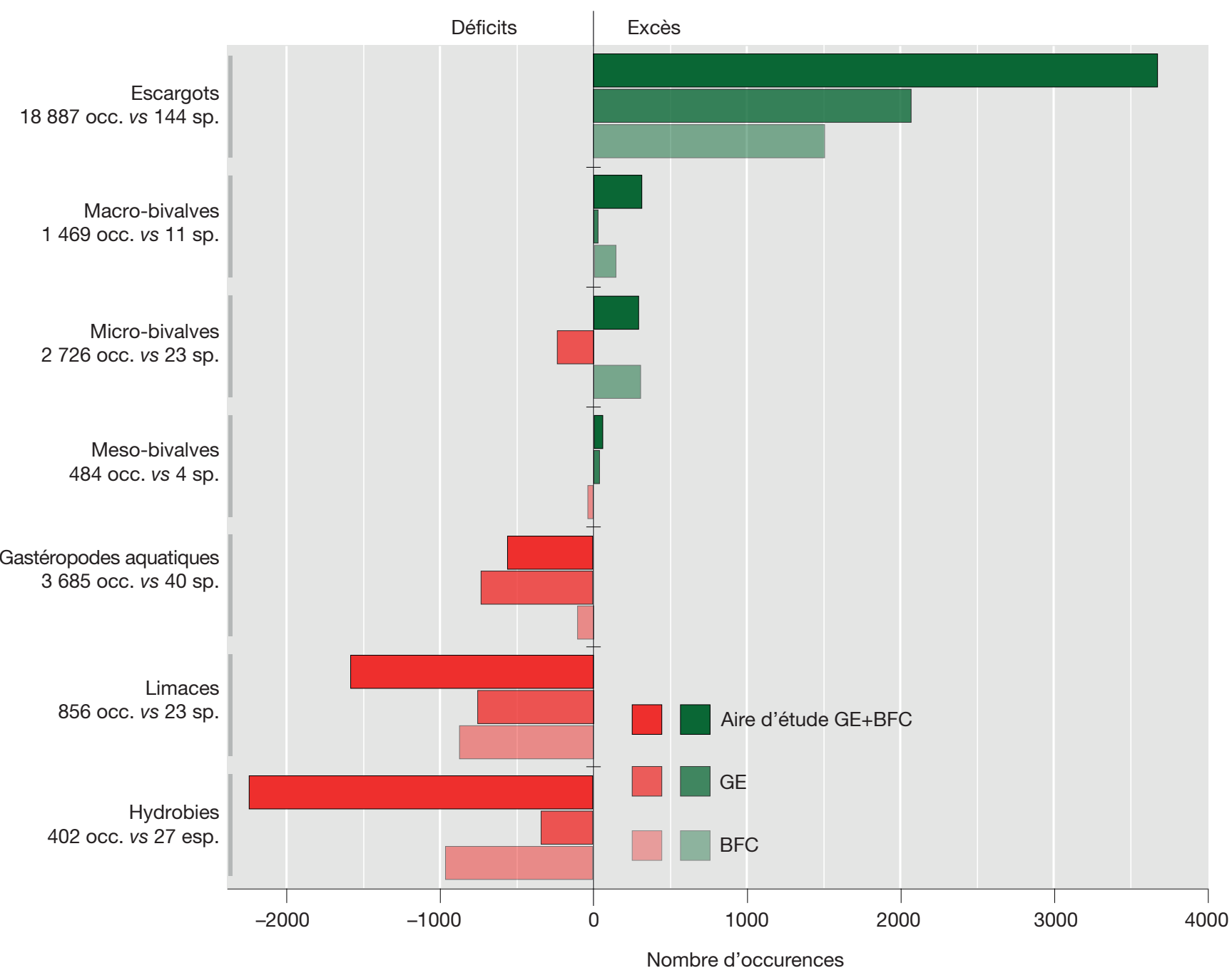

FIG. 6. - Biais taxonomiques dans les données d'occurrence. La ligne verticale $\mathrm{x}=0$ donne le nombre idéal d'occurrences par partition taxonomique quand chaque partition est échantillonnée proportionnellement à son nombre d'espèces. Les barres en rouge et en vert montrent respectivement les partitions qui sont sur- ou sous-représentées par rapport à leur échantillonnage idéal. Abréviations: BFC, Bourgogne Franche-Comté; GE, Grand Est; occ., occurences; sp., espèces.

ficativement plus faibles que celles des catégories de risque modéré (P1 vs $\mathrm{P} 3$ et $\mathrm{P} 4 ; \mathrm{P} 2$ vs $\mathrm{P} 4$; tests de Wilcoxon, $\mathrm{p}$-values $<0,05)$. Les différences ne sont pas significatives entre deux catégories de risques attenantes. Il existe par ailleurs une corrélation significative non linéaire (test de Spearman, $\mathrm{p}<0,0001, \mathrm{r}^{2} \approx 0,25$ ) entre le niveau de risque (noté de 1 à 4$)$ et le rapport $(\mathrm{M} / \mathrm{H})$ avec notamment les valeurs de $\mathrm{M} / \mathrm{H}<1$ essentiellement associées aux catégories $\mathrm{P} 1$ et $\mathrm{P} 2$. La proportion de taxons avec handicaps - ici taxonomique et/ou d'observation - est d'autant plus forte que le score global de priorité de conservation est haut.

C'est le cas, par exemple, de Bythinella carinulata (Drouët, 1867) (partition "Hydrobies») et de Truncatellina arcyensis Klemm, 1943 (partition «Escargots») qui obtiennent le score global le plus élevé ( $S G=80$; catégorie P1; rang 1 ). Cependant, le rapport $\mathrm{M} / \mathrm{H}<1$ indique des conditions défavorables pour des actions de conservation à cause ici du handicap taxonomique lié à l'ambiguïté reconnue de leur délimitation spécifique (Bank \& Neubert 2017 ; Wilke et al. 2010). Par conséquent, les actions compensatoires d'amélioration des connaissances (A1 et B1, Tableau 3) sont prioritaires sur les actions de la catégorie P1 (Tableau 2) et sur l'amélioration des moyens déployés en faveur de la conservation de ces espèces.

À l'inverse, Belgrandia gfrast Haase, 2000 (partition "Hydrobies"; SG = 75; rang 2) et Margaritifera margaritifera (Linnaeus, 1758) (partition "Macro-Bivalves"; $S G=26$; rang 6) sont deux espèces catégorisées en P1. Le rapport $\mathrm{M} / \mathrm{H}>1$ indique des conditions favorables pour le déroulement d'actions de conservation. En effet, l'unique population connue de la première espèce est située dans un espace protégé et la seconde bénéficie de fortes contraintes réglementaires nationale et européenne. En outre, leur taxonomie est stable et non remise en cause dans la littérature. Dans cette situation, les actions liées à la catégorie P1 et les actions compensatoires aux moyens déployés (C1 et D1) sont hautement nécessaires et prioritaires.

Dans le cas où $\mathrm{M} / \mathrm{H}=1$, nous recommandons d'appliquer concomitamment les actions liées aux catégories de risque ainsi que l'ensemble des actions compensatoires. 
A

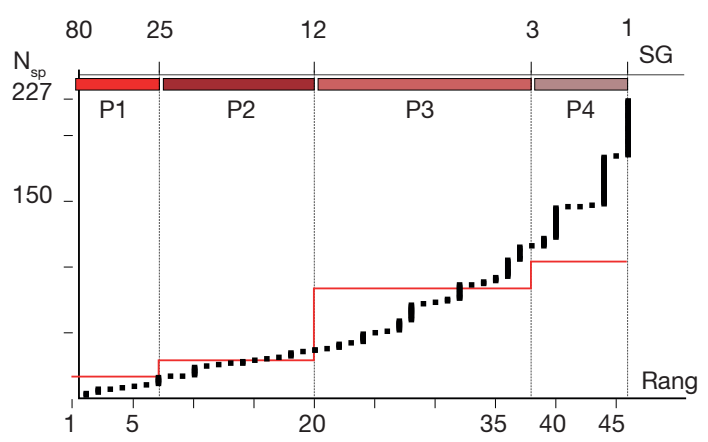

B

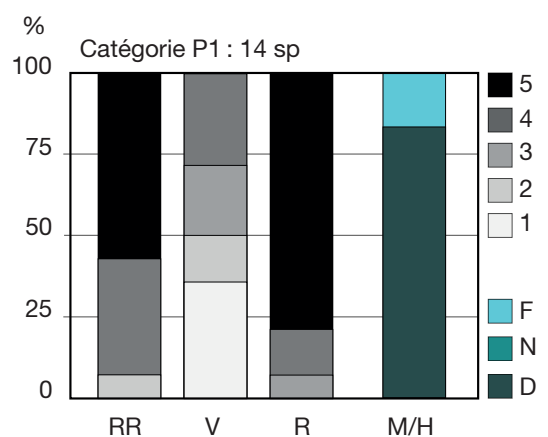

C

$\%$

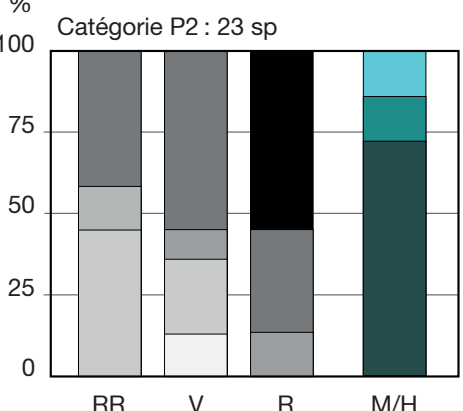

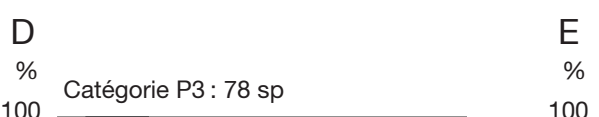
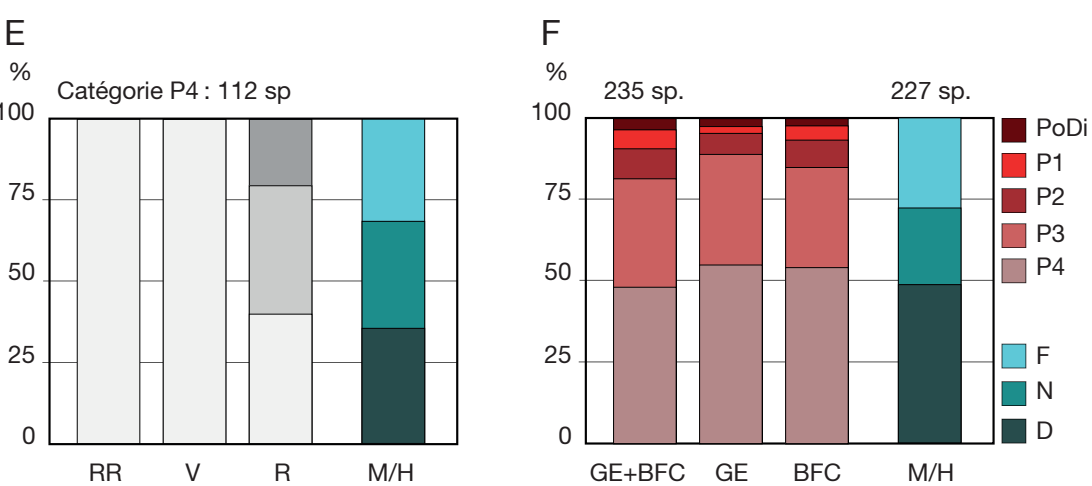

FIG. 7. - Distribution des taxons et des notes par catégories de risque. A, les points noirs donnent le nombre cumulé de taxons par rang hiérarchique, score global (SG) et catégorie de risque (P1 à P4). Les barres rouges indiquent l'effectif global dans chaque intervalle de catégorie de risque; $\mathbf{B}$ à $\mathbf{E}$, distribution des notes des taxons pour chaque critère de classification des enjeux de conservation ( $\mathbf{R}$, rareté locale; $\mathbf{R} \mathbf{R}$, responsabilité régionale; $\mathbf{V}$, vulnérabilité) et des valeurs de $\mathbf{M} / \mathrm{H}$ indiquant les moyens déployés en faveur de la conservation des espèces ( $F$ favorable pour $M / H>1, N$ neutre pour $M / H=1, D$ défavorable pour $M / H<1$ ). $M$ pour moyens déployés (note sur 5 ) et $\mathrm{H}$ pour le handicap à la conservation (note sur 5); $\mathbf{F}$, distribution des taxons par catégorie de risque pour chacune des grandes régions (BFC. Bourgogne-Franche-Comté; GE, Grand Est) et sur l'ensemble de la zone d'étude (GE + BFC). Abréviation: Nsp, nombre d'espèces par classe de note de 1 à 5.

Ainsi près de $47,6 \%\left(\mathrm{~N}_{\mathrm{sp}}=108\right)$ des espèces cibles, dont $63 \%\left(\mathrm{~N}_{\mathrm{sp}}=68\right)$ catégorisées $\mathrm{P} 1$ à $\mathrm{P} 3$, ont des scores $\mathrm{M} / \mathrm{H}$ qui nécessitent de déployer prioritairement des actions pour améliorer l'état des connaissances taxonomiques ou géographiques $(\mathrm{M} / \mathrm{H}<1)$. Il s'agit majoritairement des Hydrobies, des Limaces et des Micro-Bivalves (Fig. 8). Symétriquement, 25,2\% $\left(\mathrm{N}_{\mathrm{sp}}=30\right)$ des espèces $\mathrm{P} 1$ à $\mathrm{P} 3$ nécessitent de déployer prioritairement des actions concrètes de conservation $(\mathrm{M} / \mathrm{H} \geq 1)$. Il s'agit majoritairement des Escargots terrestres, des Gastéropodes aquatiques et des Macro-Bivalves.

La région Grand Est présente le plus grand nombre d'espèces dont la répartition globale est large, mais qui atteignent dans la région leur extrême limite de répartition occidentale. Toutes ces espèces sont catégorisées en P2 et P3 et aucune d'entre elles ne bénéficie de mesures réglementaires. La région Bourgogne-Franche-Comté présente le plus grand nombre d'espèces (micro)endémiques, toutes catégorisées en P1 ou P2, dont moins de la moitié $(42,11 \%, \mathrm{Nsp}=8)$ bénéficie de mesures réglementaires à l'échelle nationale. La quasi-totalité de ces taxons sont des Hydrobies et présentent des handicaps taxonomiques tels qu'ils ne permettent pas de rendre prioritaire les actions de conservation sur les actions d'amélioration des connaissances.

Concernant les quatre espèces qui bénéficient de contraintes réglementaires fortes nationales et européennes, Pseudunio auricularius (Spengler, 1793) (partition «Macro-Bivalves») est ici catégorisée comme disparue, Margaritifera margaritifera et Anisus vorticulus (Troschel, 1834) (partition "Gastéropodes aquatiques») sont respectivement catégorisées comme prioritaires P1 et P2. En revanche, Unio crassus présente le score global le plus faible de la catégorie $\mathrm{P} 3$, alors que quatre autres Macro-Bivalves (Potomida littoralis (Cuvier, 1798), Pseudanodonta complanata (Rossmässler, 1835), Unio tumidus Philipsson, 1788 et $U$. mancus Lamarck, 1819) présentent ici des enjeux de conservation plus forts et n'ont aucun statut réglementaire.

Les habitats aquatiques concentrent le plus grand nombre d'espèces à enjeux de conservation avec $45 \%$ d'entres elles catégorisées en $\mathrm{P} 1$ et $\mathrm{P} 2$ notamment à cause de la forte proportion en Hydrobies. Cependant, certaines d'entres elles comme Sphaerium solidum (Normand, 1844), S. rivicola (Lamarck, 1818), Euglesa pulchella (Jenyns, 1832) (partition "Micro-Bivalves») ainsi que Myxas glutinosa (O. F. Müller, 1774), Gyraulus rossmaessleri (Auerswald, 1851) et Viviparus contectus (Millet, 1813) (partition "Gastéropodes aquatiques») apparaissent avec des enjeux de conservation prioritaires. Ces taxons appartiennent à des partitions taxonomiques documentées comme déficitaires d'un point de vue des données d'occurrences ou sont, soit localisés dans les régions ayant un faible effort global d'observation, soit inféodés à des habitats eux-mêmes fortement raréfiés. Par conséquent, la priorité est ici de compenser ces handicaps par des actions d'amélioration des connaissances géographiques avant d'envisager des actions de conservation. 
A

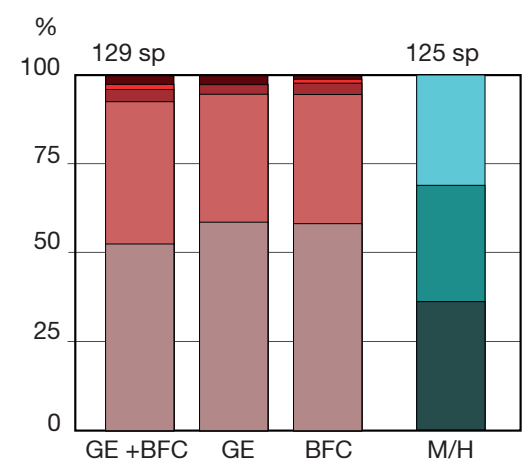

D

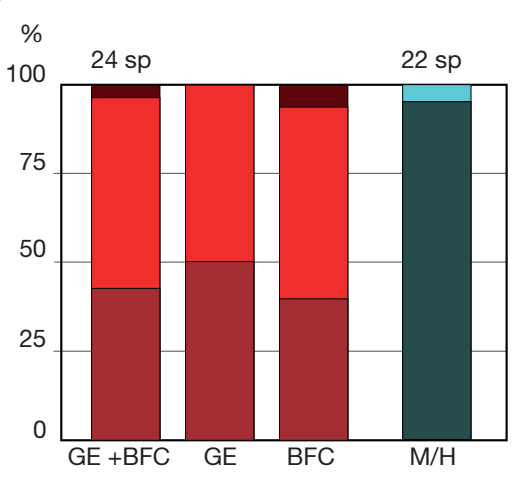

$\mathrm{G}$
B

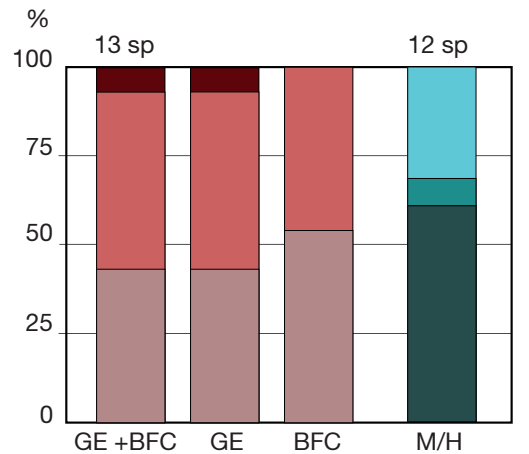

E

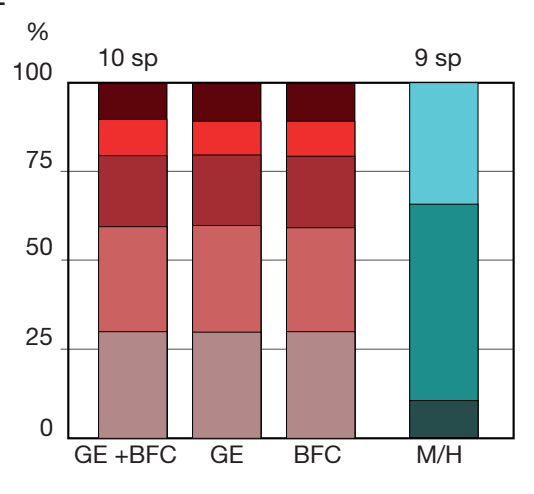

C

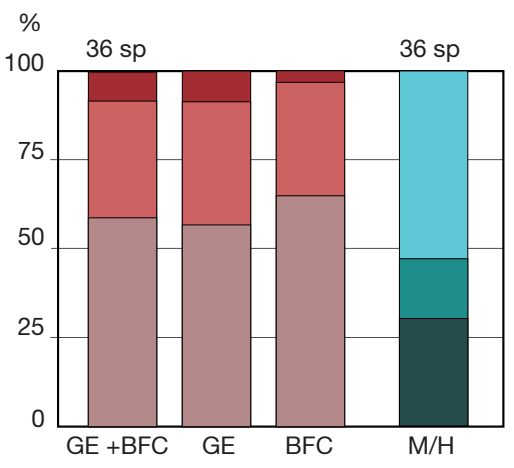

$\mathrm{F}$

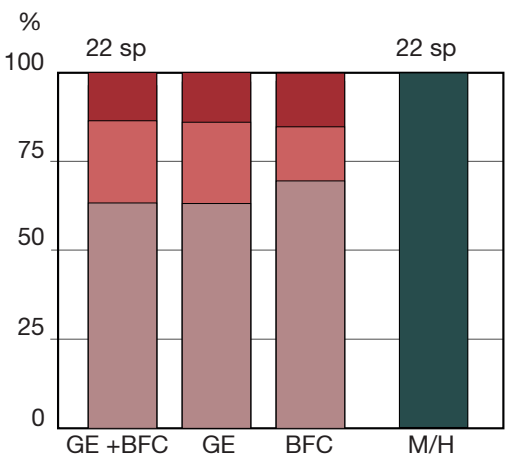

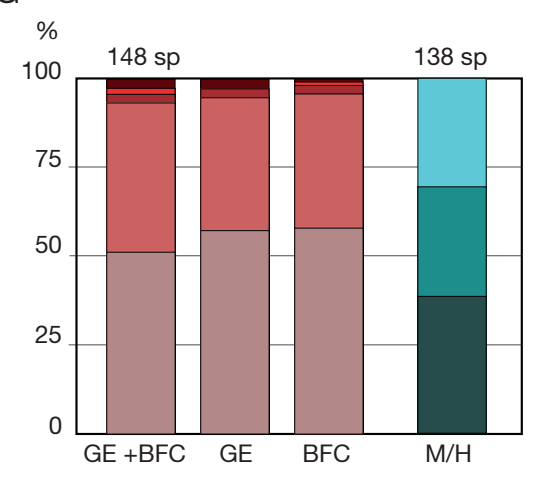

$\mathrm{H}$

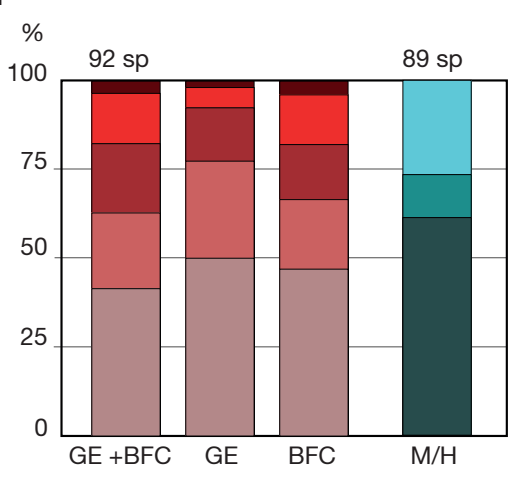

PoDi $\square$ P1 $\square$ P2 $\square$ P3 $\square$ P4 $\square$ F $\square$ N $\square \mathrm{D}$

FIG. 8. - Catégories de risque et partition taxonomique. Chaque diagramme donne la distribution du nombre de taxons par catégorie de risque pour chaque partition taxonomique pour chacune des grandes régions (BFC, Bourgogne-Franche-Comté; GE, Grand Est) sur l'ensemble de la zone d'étude (GE + BFC). Le nombre de taxon par classe de moyens déployés pour la conservation est donné pour l'ensemble de la zone d'étude. Se reporter à la Figure 7 pour les abréviations utilisées. A, Escargots; B, Limaces; C, Gastéropodes aquatiques; D, Hydrobies; E, Macro-Bivalves; F, Micro-Bivalves; G, habitats terrestres; H, habitats aquatiques.

\section{DISCUSSION}

\section{L'APPORT DES DONNÉES COLLABORATIVES}

Notre approche de hiérarchisation des enjeux de conservation repose sur un assemblage de jeux diversifiés de données d'occurrence dont près de $40 \%$ proviennent du domaine des sciences participatives (appelé aussi sciences collaboratives ou citoyennes), c'est-à-dire des données produites et transmises par des «bénévoles» (Houllier \& Merilhou-Goudard 2016). Ce type de contribution est de plus en plus considéré comme un complément significatif et indispensable à la recherche académique dédiée à la biodiversité. Groom et al. (2017) estiment que $60 \%$ des données d'observation du système mondial d'informations sur la biodiversité (GBIF : Global Biodiversity Information Facility) proviennent de programmes de science participative, alors que Chandler et al. (2017) montrent que les données collaboratives sont majoritaires pour renseigner certaines variables essentielles de biodiversité (Pereira et al. 2013) comme la répartition des espèces, l'abondance ou les changements démographiques des populations. Par ailleurs, Kosmala et al. (2016) montrent que la qualité des données produites dans le cadre de programmes participatifs peut être, en fonction de la difficulté de la tâche et de l'expérience des participants, comparable à celle des données produites par les professionnels. 
Dans notre jeu de données, nous observons que l'apport des observateurs "bénévoles" ayant des niveaux d'expertises évalués comme "faible» ou "moyen» $(34 \%)$ se concentre essentiellement sur un nombre limité de taxons faciles de détermination. La part de confiance sur les données collaboratives est donc élevée, puisque seulement $5 \%$ d'entre elles ont été écartées sur la base du critère de fiabilité taxonomique proposée par Bichain et al. (2019). Un observateur bénévole aura tendance, en cas de doute, à fournir une détermination au rang du genre ou de la famille. Cette imprécision taxonomique a pour conséquence d'écarter près de $20 \%$ des données du corpus initial d'informations, c'est-à-dire la plus grande part de l'imprécision - incluses imprécisions géographique, d'identité de l'observateur et temporelle - qui au total atteint $30 \%$. Cela indique que la maîtrise de l'application des critères diagnostiques - en d'autres termes la probabilité d'obtenir une détermination fiable à travers l'ensemble des espèces de la région - reste un point sensible de l'apport des sciences participatives dans le domaine de la malacologie continentale. Il existe pourtant un nombre d'ouvrages et de publications de plus en plus important qui permet l'identification des espèces de Mollusques continentaux. Cependant, cette littérature reste largement disséminée à travers une pléthore de revues spécialisées, la plupart en langue anglaise, limitant leur accès et leur contenu au plus grand nombre. Les outils numériques qui actuellement émergent pour l'identification des Gastéropodes terrestres sur la base d'un filtre de critères (pour exemples www.schneckenchecken.ch, dernière consultation le 30 octobre 2020 pour la faune suisse ou l'application android BiodiversiClés en France, voir Meyer et al. 2016) rendent accessibles les déterminations pour une communauté élargie d'utilisateurs. Toutefois cette démarche reste limitée ou impossible pour les taxons morphologiquement proches, et ce, en l'absence d'information concernant d'autres critères comme ceux liés à l’anatomie.

\section{BIAIS TAXONOMIQUES}

L'autre point sensible est le volume final d'informations qui est de l'ordre de 60000 données, ce qui reste modeste comparativement à d'autres groupes taxonomiques et illustre l'asymétrie considérable - quant aux informations disponibles - existant entre vertébrés et invertébrés. Dans la seule région Grand Est, les données d'observation ornithologique des portails collaboratifs VisioNature représentent près de $85 \%$ du volume total de données tous groupes taxonomiques confondus et moins de $1 \%$ pour les Mollusques. Ces ordres de grandeur sont similaires à ceux documentés par Troudet et al. (2017) à l'échelle du GBIF et confirme l'extrême biais d'observation en défaveur des invertébrés (Cardoso et al. 2011a) autant dans les programmes académiques que de sciences citoyennes. D’une manière générale, la magnitude des biais taxonomiques et géographiques, et leurs conséquences notamment sur le financement des politiques de conservation de la biodiversité, sont largement documentées (Clark \& May 2002; Seddon et al. 2005; Donaldson et al. 2016; Di Marco et al. 2017; Mammides 2019). Cardoso et al. (2011a) montrent, pour exemple, que $82 \%$ des fonds européens liés au programme
LIFE-Nature sont dirigés vers les vertébrés alors qu'ils ne représentent au mieux que $7 \%$ de la faune et de la flore globales. Les Arthropodes, les Mollusques et les Plantes se partagent la part restante avec en moyenne $1850 €$ dédiés par espèce $v$ $230000 €$ par espèce de Vertébré. Ce déséquilibre financier est souvent justifié par les décideurs et les administrateurs politiques sur l'idée que certains vertébrés considérés comme "espèces parapluies" protègent indirectement les Invertébrés qui occupent le même habitat (Heywood et al. 1995; Meffe \& Carroll 1997; Simberloff 1998). Cependant, cette stratégie de conservation est dans la majorité des cas non évaluée et se révèle souvent inefficace quand elle est testée (Roberge \& Angelstam 2004; Fontaine et al. 2007; Runge et al. 2019).

L'origine des biais taxonomiques dans les programmes de recherche semble plus reposer sur des préférences sociétales que sur des critères objectifs fondés sur les réel(le)s enjeux/ priorités de conservation ou de connaissance (Troudet et al. 2017). Plusieurs facteurs ont été avancés pour expliquer ces préférences, tels que l'esthétique, la taille du corps, le sentiment de vulnérabilité ou de rareté mais aussi la proximité phylogénétique (Miralles et al. 2019).

Les biais fonctionnent aussi à l'intérieur d'un même groupe taxonomique (voir par exemple Dos Santos et al. 2020), ce que nous observons par ailleurs dans notre jeu de données avec un déficit global d'occurrences et d'observations pour les groupes informels des Limaces et des Gastéropodes aquatiques avec notamment les Hydrobies. Concernant ces dernières, il faudrait multiplier l'effort d'échantillonnage jusqu'à 40 fois, afin de disposer d'un jeu de données comparable aux groupes les mieux échantillonnés comme les Gastéropodes terrestres ou les Macro-Bivalves. Or, les Hydrobies représentent ici $62 \%$ des espèces protégées par la loi française et $43 \%$ des espèces catégorisées comme menacées dans les Listes rouges mondiales et européennes de l'UICN. Par ailleurs, Böhm et al. (2020) estiment que les deux tiers $(68,7 \%)$ des espèces du groupe présentent un risque d'extinction à plus ou moins court terme.

Nous montrons que plusieurs facteurs opèrent dans l'origine de ces biais taxonomiques avec notamment: la rareté intrinsèque des espèces, le handicap taxonomique c'est-à-dire la difficulté d'identifier les taxons et/ou la fiabilité de leurs descripteurs et les mesures réglementaires contraignantes qui orientent la recherche des espèces. En revanche, nous n'avons pas testé l'hypothèse de préférence sociétale liée par exemple à des espèces connues du grand public (Escargot de bourgogne Helix pomatia Linnaeus, 1758, Petit gris Cornu aspersum (O. F. Müller, 1774)), ou charismatiques comme la Mulette perlière Margaritifera margaritifera.

Concernant les Hydrobies, ce sont les facteurs de rareté intrinsèque et de handicap taxonomique qui opèrent majoritairement dans le déficit de connaissance et par conséquence probablement dans l'absence de déploiement de mesures adaptées de conservation. À l'inverse, l'excès d'observations en faveur des Macro-Bivalves est lié à la surreprésentation d'informations concernant la Mulette épaisse (Unio crassus). L'espèce bénéfice en effet d'une double réglementation française et européenne (les mesures réglementaires) qui permet finalement d'obtenir un état satisfaisant des connaissances 
sur sa répartition géographique, sans toutefois apporter des éléments sur l'état de la vitalité des populations (Lamand \& Cucherat 2021). En effet, les données dans ce cas précis ne sont que de nature qualitative et n'apportent pas d'élèments sur la dynamique des populations. Un déficit d'informations limite donc la connaissance de la répartition, mais aussi de l'état de conservation, des populations des autres Macro-Bivalves qui ne bénéficient pas de la même pression d'observations.

\section{CONTRAINTE DU CHOIX DE LA MÉTHODE} DE HIÉRARCHISATION

D’une manière générale, Cuttelod et al. (2011) estiment que 82,6 \% des Mollusques d'eau douce et $53 \%$ des terrestres ne sont pas documentés sur la taille des effectifs et sur la dynamique de leurs populations. Conséquemment, les catégorisations UICN sont construites majoritairement à partir des uniques critères liés à l'estimation des zones d'occupation (AOO) ou d'occurrence (EOO) et des menaces qui ont pesé, qui agissent ou qui pourraient agir sur les espèces. Par ailleurs, les traits de vie des espèces et leur sensibilité aux pressions sur leur(s) habitat(s) restent largement méconnus ou approximés à travers quelques espèces mieux étudiées. Ainsi, l'absence de ces informations sur la dynamique des populations est un handicap majeur dans l'évaluation des enjeux de conservation des Mollusques continentaux, qui sont considérés par ailleurs comme particulièrement vulnérables et menacés (Lydeard et al. 2004; Geist \& Auerswald 2007 ; Cuttelod et al. 2011; Régnier et al. 2015; Cowie et al. 2017; Böhm et al. 2020). Le problème de fond reste la priorisation de l'allocation des ressources vers des actions possibles de conservation sur la base d'une méthodologie explicite et reproductible, ce que ne permettent pas ni les Listes rouges, ni les listes d'espèces protégées (Le Berre et al. 2019). La rareté des informations sur les populations, leur autoécologie, leurs traits de vie ou leurs habitat/microhabitat réduit considérablement le choix des méthodes de priorisation/hiérarchisation. Or, la méthode proposée par Gauthier et al. (2010), laquelle repose sur seulement trois critères - la responsabilité régionale, la vulnérabilité et la rareté locale - peut être suffisante et pertinente pour déterminer les priorités de conservation, même si d'autres choix sont possibles en fonction des objectifs et du contexte local (Le Berre et al. 2019). En outre, Dunn et al. (1999) soulignent que les différences obtenues entre les systèmes de classement sont moins importantes que la nécessité de mettre en œuvre des processus d'évaluation pour identifier et fournir des priorités et des objectifs bien définis de stratégies afin d'améliorer l'efficacité des actions de conservation.

Notre démarche a donc été de proposer, à partir du classement obtenu en suivant la méthode de Gauthier et al. (2010), des catégories indicatives de la possible extinction globale ou régionale des taxons ou de réduction de leur aire de répartition. Nous considérons que l'enjeu de conservation d'un taxon est d'autant plus prioritaire que le risque d'extinction, global ou partiel, est élevé. Les actions associées à chaque catégorie, et donc de priorité, sont essentiellement ciblées sur l'amélio- ration des connaissances concernant la répartition et l'état des populations ainsi que sur les pressions qui s'exercent sur les habitats. Cela concerne près de $51 \%$ des espèces cibles $\left(\mathrm{N}_{\mathrm{sp}}=227\right)$ de l'étude dont 37 sont classées dans les catégories hautement prioritaires (P1 et P2).

L'évaluation de l'état de conservation des espèces de Mollusques continentaux est limitée par la qualité des données saisies dans les bases de données naturalistes. Au-delà des saisies d'occurrence, supposant une identification correcte de l'espèce, le remplissage d'informations connexes permettrait d'améliorer l'exercice d'évaluation. Comme piste de réflexion, ces informations devraient documenter au moins le statut des animaux observés vivants $v$ s non vivants et des informations minimales sur l'abondance estimée des populations concernées. Ces informations qui restent à bien définir, pour un usage précis et non équivoque, permettraient d'avoir une évaluation en première approche sur l'état des populations.

\section{TAXONOMIE ET HANDICAP À LA HIÉRARCHISATION} DES ENJEUX DE CONSERVATION

La fiabilité de la hiérarchisation ici proposée est directement dépendante de la quantité et de la qualité des informations disponibles notamment taxonomiques. En effet, la méconnaissance taxonomique est marquée dans le groupe des Hydrobies pour lequel les délimitations spécifiques sont encore largement discutées. À titre d'exemple, Richling et al. (2017) montrent que les 14 taxons nominaux du genre Bythiospeum Bourguignat, 1882 - présents dans l'est de la France, en Suisse et dans le sud-ouest de l'Allemagne - appartiennent en réalité à cinq lignées moléculaires attribuables au rang d'espèce. Ces auteurs posent comme hypothèse qu'il n'y aurait que trois espèces au lieu des 25 taxons nominaux listées dans la Liste rouge des Mollusques menacées en Allemagne (Jungluth \& Knorre 2012). La conséquence n'est pas anodine, puisqu'il s'agirait d'un changement radical de vue sur l'extrême diversité du groupe et de leur enjeu de conservation. Leur conclusion est similaire à celle des autres études menées dans le genre Bythinella Moquin-Tandon, 1855 (Bichain et al. 2007; Haase et al. 2007; Wilke et al. 2010) qui soulignent aussi que les caractères de la coquille et anatomiques sont de mauvais indicateurs de la délimitation des espèces. Ces deux genres représentent $62,5 \%\left(\mathrm{~N}_{\mathrm{sp}}=15\right)$ des espèces d'Hydrobies de notre étude, et sont toutes placées dans les plus hautes catégories d'enjeux de conservation.

À l'inverse, pour les Limaces, Rowson et al. (2014) montrent que les approches taxonomiques fondées sur les caractères anatomiques sous-estiment significativement - pour la faune britannique et irlandaise - le nombre réel d'espèces de l'ordre de $20 \%$ dont une part de taxons non-décrits. Concernant les Gastéropodes terrestres, Truncatellina arcyensis est classée dans les enjeux prioritaires de conservation (rang 1 sur 22). Ce qui est fondé sur l'opinion taxonomique de Falkner et al. (2002), repris dans TAXREF v13 (Gargominy et al. 2019), qui considèrent cette espèce comme un endémique français dont l'aire de répartition est limitée au seul département de Côte-d'Or. L'espèce est par ailleurs catégorisée en danger 
d'extinction sur les Listes rouges mondiale et européenne de l'UICN et inscrite à l'article 4 de l'arrêté de 2007 fixant les listes des Mollusques protégés en France. Or, l'espèce est traitée par Bank \& Neubert (2017) comme un synonyme plus ancien de Truncatellina cylindrica (A. Férussac, 1807), sur la base des résultats présentés par Holyoak et al. (2012) et qui reposent sur une approche uniquement morphométrique. T. cylindrica est une espèce à large répartition qui ne présente pas d'enjeux de conservation dans notre classification (rang 20 sur 22). Cette situation est emblématique des conflits d'experts qui opèrent dans la taxonomie des Mollusques continentaux. Aujourd'hui aucune révision taxonomique ne permet de statuer sur la validité de cette espèce. D'autres taxons (re)validés par Falkner et al. (2002) ont récemment été mis en synonymie à travers des approches de taxonomie intégrative comme Euconulus callopisticus (Bourguignat, 1880), E. praticola (Reinhardt, 1883) et E. trochiformis (Montagu, 1803) par Horsáková et al. (2019) et Horsáková et al. (2020) ou Pupilla bigranata (Rossmässler, 1839) par Balashov et al. (2019). Globalement, près de 20 ans se sont écoulées entre validation $a d$ hoc et révision taxonomique de ces espèces.

À l'inverse, Araujo et al. (2005) démontrent la validité taxonomique d'Unio mancus, espèce longtemps enfouie dans le fourre-tout synonymique des années 1980 et qui répond aux critères qui permettraient de la catégoriser comme «En danger» dans la Liste rouge de l'UICN (Prié et al. 2012). Ce niveau de catégorisation est équivalant à celui d'Unio crassus, dont le concept actuel de délimitation pourrait en réalité inclure deux espèces distinctes (Prié 2017), ce qui remettrait en question les contours de sa conservation.

L'ensemble de ces exemples illustre l'interdépendance taxonomie-conservation, problématique largement discutée par ailleurs (May 1990; Dubois 2003; Mace 2004; Pillon \& Chase 2007; Morrison et al. 2009; Copus et al. 2018). D’une manière générale, nous montrons que près d'une espèce cible sur deux $\left(49 \%, \mathrm{~N}_{\mathrm{sp}}=108\right)$ présente un handicap de connaissance majeur soit taxonomique, d'identification et/ou de répartition. Nous assumons donc le fait que les difficultés objectives dans le processus de hiérarchisation, liées à la mauvaise qualité de l'information, peuvent conduire à fausser les enjeux réels de conservation et leur priorité afférente. Par conséquent, nous recommandons pour tous les taxons classés prioritaires, mais dont le niveau de handicap se révèle élevé, de dérouler avant tout un programme ciblé d'actions d'amélioration des connaissances.

Globalement, nous estimons qu'il serait nécessaire de revisiter les limites interspécifiques par des approches intégratives (Dayrat 2005) de 29 taxons nominaux dont $48 \%$ sont placés dans la plus haute catégorie de menace et d'enjeux de conservation. Par ailleurs, nous identifions 27 espèces cibles pour lesquelles l'identification, pour être fiable, nécessite soit une approche purement moléculaire $\left(\mathrm{N}_{\mathrm{sp}}=4\right)$, soit l'examen de leur caractère anatomique $\left(\mathrm{N}_{\mathrm{sp}}=23\right)$. C'est le cas notamment chez les Lymnées s.l. (Gastéropodes aquatiques) dont les délimitations génériques et spécifiques ont été récemment revisitées (Aksenova et al. 2018) et où il apparaît que certaines espèces ne peuvent être identifiées qu'à partir d'une approche type barcode (Hebert et al. 2003). Enfin, au moins 28 taxons ont des niches écologiques et des (micro)habitats restreints, difficilement accessibles, impliquant pour leur recherche le déploiement de méthode comme celle de l'ADN environnemental.

De la même manière, pour les huit espèces ici jugées comme potentiellement disparues, il est nécessaire de combler les lacunes de connaissances géographiques avant de statuer définitivement sur leur extinction à l'échelle régionale.

\section{CONCLUSIONS}

Nous montrons que $51 \%$ des espèces cibles $\left(\mathrm{N}_{\mathrm{sp}}=119\right)$ ont un volume et une qualité d'informations jugés satisfaisants afin d'évaluer de manière fiable leur niveau de priorisation. Il apparaît que 47 d'entre elles présentent des enjeux de conservation prioritaires (catégories P1 à P3) dont neuf hautement prioritaires (catégories P1 \& P2). Seules sept de ces espèces bénéficient d'une réglementation à travers la Directive Européenne Habitat-Faune-Flore et/ou sont protégées en France dont Margaritifera margaritifera, Anisus vorticulus et Vertigo genesii (Gredler, 1856), classées dans les catégories hautement prioritaires. En revanche, les quatre autres (Vertigo moulinsiana (Dupuy, 1849), V. angustior Jeffeys, 1830, V. geyeri Lindholm, 1925 et Unio crassus) sont classées dans la catégorie intermédiaire P3 de moindre priorité. Par ailleurs, 24 espèces ont au moins $25 \%$ de leur aire de répartition connue qui est incluse dans un espace à forte contrainte réglementaire dont seulement deux espèces de la Directive européenne, aucune ne bénéfice d'un statut français de protection. Enfin $43 \%$ des espèces classées dans les plus hautes catégories de menace sont par ailleurs catégorisées comme menacées dans les Listes rouges de l'UICN européennes.

Nous montrons donc qu'il n'existe pas actuellement de bons indicateurs pour le législateur ou le conservateur afin d'identifier, évaluer et prioriser les besoins de conservation à l'échelle régionale. Les espèces inscrites sur les Listes rouges, protégées ou réglementées représentent à peine la moitié ou le dixième des espèces classées respectivement dans les plus hautes ou de moindres catégories de priorité. La prise en compte de ces espèces à travers les outils réglementaires n'est donc pas représentative des besoins réels de conservation pour les Mollusques continentaux dès lors que $50 \%$ d'entre eux présentent un risque de régression significative de leur répartition, et près de $15 \%$ un risque majeur d'extinction à l'échelle régionale. Actuellement, aucune espèce de Mollusque ne bénéficie d'un plan d'actions de conservation régionale dans l'une des deux régions de l'étude. Seules les espèces de la Directive européenne bénéficient ponctuellement de financements pour des programmes d'amélioration des connaissances, de gestion via les contrats Natura 2000 ou pour la rédaction des documents d'objectifs. Enfin certaines espèces protégées peuvent bénéficier de mesures compensatoires.

La liste hiérarchisée que nous proposons ici permet de poser les premières bases d'une stratégie régionale pour la conservation et l'amélioration des connaissances des Mollusques continentaux. Le premier axe de cette stratégie est d'améliorer prioritairement les connaissances taxonomiques (domaine 
des sciences académiques) et géographiques (domaine des sciences participatives) pour les espèces prioritaires identifiées comme ayant un handicap, taxonomique ou géographique, à la conservation. Pour les espèces prioritaires identifiées comme n'ayant pas de handicap, le deuxième axe serait:

- d'évaluer les effectifs, les dynamiques et la répartition des populations;

- d'appliquer des mesures conservatoires ou compensatoires adaptées dans les espaces réglementées et/ou sous gestion;

- d'appliquer une réglementation régionale pour les espèces ou les espaces concernés.

À partir des faiblesses concernant les aspects quantitatifs et qualitatifs des informations ici mises en évidence, notre travail permet de dégager un schéma général afin d'améliorer les procédures d'évaluation et de priorisation des enjeux de conservation. D'une part, avec la consolidation des connaissances taxonomiques, la construction et l'accessibilité d'outils diagnostiques vers le plus grand nombre d'observateurs. D'autre part, avec l'amélioration des connaissances géographiques, écologiques et populationnelles des espèces par un travail de modification et d'harmonisation des bases de données collaboratives existantes, qui aujourd'hui semblent ne répondre que partiellement aux enjeux environnementaux.

Enfin, il semble indispensable de (re)connecter les différents espaces et acteurs liés à l'étude et à la conservation de la biodiversité - recherche fondamentale et appliquée, milieu associatif, décideurs et gestionnaires - afin de permettre une meilleure intégration des produits de la recherche dans la gouvernance et la gestion des milieux naturels et ainsi aboutir à des stratégies de conservation mieux définies face à la réalité des menaces.

\section{Remerciements}

Ce travail s'inscrit dans le cadre des études menées par le Groupe d'Étude de Malacologie de la Société d'Histoire naturelle et d'Ethnographie de Colmar (SHNEC). Les auteurs remercient ici la SHNEC pour les locaux et le matériel mis à leur disposition. Les auteurs adressent par ailleurs tous leurs remerciements aux fournisseurs de données et ceux/celles qui ont facilité ce travail d'une manière ou d'une autre et particulièrement à Emmanuel Fara (Université de Bourgogne) pour sa relecture attentive du mansucrit, à Florent Lamand pour la transmission des données de l'Office français de la Biodiversité et à Solène Robert pour la transmission des données de la plateforme INPN/SINP. Nos remerciements s'adressent également aux deux évaluateurs, Maëlle Le Berre et Francis Olivereau, pour leurs commentaires et apports constructifs et pertinents.

Enfin, nous tenons à remercier les membres de la communauté scientifique qui, par tous les moyens actuels, permettent un accès libre et gratuit à leurs publications. Nous remercions particulièrement Alexandra Elbakyan (Sci-Hub) ainsi que les gestionnaires du site ResearchGate pour leur contribution significative à la démocratisation des produits de la recherche.

\section{RÉFÉRENCES}

Aksenova O. V., Bolotov I. N., Gofarov M. Y., Kondakov A. V., Vinarski M. V., Bespalaya Y. V., Kolosova Y. S., Palatov D. M., Sokolova S. E., Spitsyn V. M., Tomilova A. A., Travina O. V. \& VikhreV I. V. 2018. - Species richness, molecular taxonomy and biogeography of the radicine pond snails (Gastropoda: Lymnaeidae) in the Old World. Scientific Reports 8 (1): 1-17. https://doi.org/10.1038/s41598-018-29451-1

Araujo R., Gómez I. \& Machordom A. 2005. — The identity and biology of Unio mancus Lamarck, 1819 ( = U. elongatulus) (Bivalvia: Unionidae) in the Iberian peninsula. Journal of Molluscan Studies 71 (1): 25-31. https://doi.org/10.1093/mollus/eyi002

Balashov I., Neiber M. T., Bogon K. \& Hausdorf B. 2019. On the identity of "Pupilla bigranata" populations from Germany and Ukraine (Gastropoda: Pupillidae). Archiv für Molluskenkunde International Journal of Malacology 148 (1): 1-7. https://doi. org/10.1127/arch.moll/148/001-007

BANK R. A. \& NeUBERT E. 2017. - Checklist of the Land and Freshwater Gastropoda of Europe [en ligne]. http://www.marinespecies. org/aphia.php? $=$ =sourcedetails\&id $=279050$

Bichain J.-M., GAUBERT P., SAMADI S. \& BOISSELIER-Dubayle M.-C. 2007. - A gleam in the dark: phylogenetic species delimitation in the confusing spring-snail genus Bythinella Moquin-Tandon, 1856 (Gastropoda: Rissooidea: Amnicolidae). Molecular Phylogenetics and Evolution 45 (3): 927-941. https://doi.org/10.1016/j. ympev.2007.07.018

Bichain J.-M., Cucherat X., Brulé H., Durr T., Guhring J., Hommay G., Ryelandt J. \& Umbrecht K. 2019. — Liste de référence fonctionnelle et annotée des Mollusques continentaux (Mollusca: Gastropoda \& Bivalvia) du Grand-Est (France). Naturae 2019 (11). https://doi.org/10.5852/naturae2019a11

BÖHM M., DeWhurst-Richman N. I., SedDON M., Ledger S. E. H., Albrecht C., Allen D., Bogan A. E., Cordeiro J., Cummings K. S., Cuttelod A., Darrigran G., Darwall W., Fehér Z., Gibson C., Graf D. L., Köhler F., Lopes-Lima M., Pastorino G., Perez K. E., Smith K., Van Damme D., Vinarski M. V., Von Proschwitz T., Von Rintelen T., Aldridge D. C., Aravind N. A., Budha P. B., Clavijo C., Van Tu D., Gargominy O., Ghamizi M., HaAse M., HilTON-Taylor C., Johnson P. D., KebapÇi Ü., Lajtner J., Lange C. N., Lepitzki D. A. W., Martínez-Ortí A., Moorkens E. A., Neubert E., Pollock C. M., Prié V., Radea C., Ramirez R., Ramos M. A., Santos S. B., Slapnik R., Son M. O., StensGaARD A.-S. \& Collen B. 2020. - The conservation status of the world's freshwater molluscs. Hydrobiologia. https:// doi.org/10.1007/s10750-020-04385-w

Bonnet X., Shine R. \& Lourdais O. 2002. - Taxonomic chauvinism. Trends in Ecology \& Evolution 17 (1): 1-3. https://doi. org/10.1016/S0169-5347(01)02381-3

BotTrill M. C., Joseph L. N., CARWARdine J., Bode M., CoOK C., Game E. T., Grantham H., Kark S., Linke S., McDonaldMadden E., Pressey R. L., Walker S., Wilson K. A. \& PosSINGHAM H. P. 2008. - Is conservation triage just smart decision making? Trends in Ecology \& Evolution 23 (12): 649-654. https:// doi.org/10.1016/j.tree.2008.07.007

Cardoso P., ERWin T. L., Borges P. A. V. \& NeW T. R. 2011 a. The seven impediments in invertebrate conservation and how to overcome them. Biological Conservation 144 (11): 2647-2655. https://doi.org/10.1016/j.biocon.2011.07.024

Cardoso P., Borges P. A. V., Triantis K. A., Ferrández M. A. \& Martín J. L. 2011 b. - Adapting the IUCN Red List criteria for invertebrates. Biological Conservation 144 (10): 2432-2440. https://doi.org/10.1016/j.biocon.2011.06.020

Cardoso P., Borges P. A. V., Triantis K. A., Ferrández M. A. \& MARTíN J. L. 2012. - The underrepresentation and misrepresentation of invertebrates in the IUCN Red List. Biological Conservation 149 (1): 147-148. https://doi.org/10.1016/j.biocon.2012.02.011 
CARLTON J. T. 1996. - Biological invasions and cryptogenic species. Ecology 77 (6): 1653-1655. https://doi.org/10.2307/2265767

Chandler M., See L., Copas K., Bonde A. M. Z., López B. C., Danielsen F., Legind J. K., Masinde S., Miller-Rushing A. J., Newman G., Rosemartin A. \& Turak E. 2017. — Contribution of citizen science towards international biodiversity monitoring. Biological Conservation 213 (Part B): 280-294. https:// doi.org/10.1016/j.biocon.2016.09.004

Chapman A. D. 2009. - Numbers of Living Species in Australia and the World. 2nd edition. Australian Biodiversity Information Services, Toowoomba, $84 \mathrm{p}$.

ClARK J. A. \& MAY R. M. 2002. - Taxonomic bias in conservation research. Science 297 (5579): 191-192. https://doi.org/10.1126/ science.297.5579.191b

Colwell R. K., Chao A., Gotelli N. J., Lin S.-Y., MaO C. X., Chazdon R. L. \& Longino J. T. 2012. - Models and estimators linking individual-based and sample-based rarefaction, extrapolation and comparison of assemblages. Journal of Plant Ecology 5 (1): 3-21. https://doi.org/10.1093/jpe/rtr044

Copus J. M., Montgomery W. L., Forsman Z. H., Bowen B. W. \& TOONEN R. J. 2018. - Geopolitical species revisited: genomic and morphological data indicate that the roundtail chub Gila robusta species complex (Teleostei, Cyprinidae) is a single species. Peer] 6: e5605. https://doi.org/10.7717/peerj.5605

Cowie R. H., Régnier C., Fontaine B. \& Bouchet P. 2017. Measuring the Sixth Extinction: what do mollusks tell us? The Nautilus 131 (1): 3-41.

Cuttelod A., Seddon M. B. \& Neubert E. 2011. - European Red List of non-Marine Molluscs. European Commission, Luxembourg, 97 p. https://doi.org/10.2779/84538

DAYRAT B. 2005. - Towards integrative taxonomy. Biological Journal of the Linnean Society 85 (3): 407-417. https://doi. org/10.1111/j.1095-8312.2005.00503.x

Di Marco M., Chapman S., Althor G., Kearney S., Besancon C., Butt N., Maina J. M., Possingham H. P., Rogalla von BieBerstein K., Venter O. \& Watson J. E. M. 2017. — Changing trends and persisting biases in three decades of conservation science. Global Ecology and Conservation 10: 32-42. https://doi. org/10.1016/j.gecco.2017.01.008

Donaldson M. R., Burnett N. J., Braun D. C., Suski C. D., Hinch S. G., CoOKe S. J. \& KerR J. T. 2016. - Taxonomic bias and international biodiversity conservation research. FACETS 1 (1): 105-113. https://doi.org/10.1139/facets-2016-0011

Dos Santos J. W., Correia R. A., Malhado A. C. M., CamposSilva J. V., Teles D., Jepson P. \& Ladle R. J. 2020. - Drivers of taxonomic bias in conservation research: a global analysis of terrestrial mammals. Animal Conservation 23 (6): 678-688 https://doi.org/10.1111/acv.12586

DubOIS A. 2003. - The relationships between taxonomy and conservation biology in the century of extinctions /Les relations entre la taxinomie et la biologie de la conservation au siècle des extinctions. Comptes Rendus Biologies 326 (Suppl. 1): 9-21. https:// doi.org/10.1016/S1631-0691(03)00022-2

Dunn E. H., David J. T. Hussell \& Welsh D. A. 1999. — Prioritysetting tool applied to canada's landbirds based on concern and responsibility for species. Conservation Biology 13 (6): 1404-1415. https://doi.org/10.1046/J.1523-1739.1999.98400.X

Eisenhauer N., Bonn A. \& Guerra C. A. 2019. - Recognizing the quiet extinction of invertebrates. Nature Communications 10 (1): 50. https://doi.org/10.1038/s41467-018-07916-1

ERWIN T. L. 1982. - Tropical forests: their richness in Coleoptera and other arthropod species. The Coleopterists Bulletin 36 (1): 74-75.

FALKNER G., RipKEN T. E. J. \& FALKNER M. 2002. - Mollusques continentaux de France. Liste de référence annotée et Bibliographie. Muséum national d'Histoire naturelle (Coll. Patrimoines naturels; 52), Paris, 356 p.

Fontaine B., Gargominy O. \& Neubert E. 2007. — Priority sites for conservation of land snails in Gabon: testing the umbrella species concept. Diversity and Distributions 13 (6): 725-734. https://doi.org/10.1111/j.1472-4642.2007.00376.x

Game E. T., Kareiva P. \& Possingham H. P. 2013. - Six common mistakes in conservation priority setting. Conservation Biology 27 (3): 480-485. https://doi.org/10.1111/cobi.12051

Gargominy O., Prié V., Bichain J.-M., Cucherat X. \& FonTAINE B. 2011. - Liste de référence annotée des mollusques continentaux de France. MalaCo 7: 307-382.

Gargominy O., Tercerie C., Régnier C., Ramage T., Dupont P., DASZKIEWICZ P. \& PONCET L. 2019. - TAXREF v13, référentiel taxonomique pour la France: méthodologie, mise en cuvre et diffusion Rapport Patrinat. UMS PatriNat (OFB-CNRS-MNHN), Paris, 63 p.

Gauthier P., Debussche M. \& Thompson J. D. 2010. — Regional priority setting for rare species based on a method combining three criteria. Biological Conservation 143 (6): 1501-1509. https:// doi.org/10.1016/j.biocon.2010.03.032

Geist J. \& Auerswald K. 2007. — Physicochemical stream bed characteristics and recruitment of the freshwater pearl mussel (Margaritifera margaritifera). Freshwater Biology 52 (12): 22992316. https://doi.org/10.1111/j.1365-2427.2007.01812.x

GLÖ̈R P. 2019. - The Freshwater Gastropods of the West Palaearctis Vol. 1. Biodiversity Research Lab, Hetlingen, 399 p.

Godet L. \& DeVictor V. 2018. - What conservation does. Trends in Ecology \& Evolution 33 (10): 720-730. https://doi. org/10.1016/j.tree.2018.07.004

GRAF D. L. 2013. - Patterns of freshwater bivalve global diversity and the state of phylogenetic studies on the Unionoida, Sphaeriidae, and Cyrenidae. American Malacological Bulletin 31 (1): 135-153. https://doi.org/10.4003/006.031.0106

GroOM Q., WEATHERDON L. \& GEIJZENDORFFER I. R. 2017. Is citizen science an open science in the case of biodiversity observations? Journal of Applied Ecology 54 (2): 612-617. https:// doi.org/10.1111/1365-2664.12767

HAASE M., WILKE T. \& MiLDNER P. 2007. — Identifying species of Bythinella (Caenogastropoda: Rissooidea): a plea for an integrative approach. Zootaxa 1563 (1): 1-16. https://doi.org/10.11646/ zootaxa.1563.1.1

Hebert P. D. N., Cywinska A., Ball S. L. \& DeWaArd J. R. 2003. - Biological identifications through DNA barcodes. Proceedings of the Royal Society of London. Series B: Biological Sciences 270 (1512): 313-321. https://doi.org/10.1098/rspb.2002.2218

HeYwood V. H., Watson R. T. \& Programme U. N. E. 1995. Global Biodiversity Assessment.Cambridge; New York, NY, USA Cambridge University Press., 1140 p.

HolyOAK D. T., HOlYOAK G. A. \& TORRES J. S. 2012. - A reassessment of the species of Truncatellina (Gastropoda: Vertiginidae) in the Iberian Peninsula and North-west Africa. Iberus 30 (2): 7-33.

HorsáKOVÁ V., NeKOla J. C. \& HorSÁK M. 2019. — When is a "cryptic" species not a cryptic species: a consideration from the Holarctic micro-landsnail genus Euconulus (Gastropoda: Stylommatophora). Molecular Phylogenetics and Evolution 132: 307-320. https://doi.org/10.1016/j.ympev.2018.12.004

HorsáKOVÁ V., NeKOla J. C. \& HorsÁK M. 2020. — Integrative taxonomic consideration of the Holarctic Euconulus fulvus group of land snails (Gastropoda, Stylommatophora). Systematics and Biodiversity 18 (2): 142-160. https://doi.org/10.1080/1477200 0.2020 .1725172

Houllier F. \& Merilhou-Goudard J.-B. 2016. — Les sciences participatives en France. État des lieux, bonnes pratiques et recommandations. INRAE, Paris, 63 p. https://doi.org/10.15454/1.4 606201248693647E12

Jungluth J. H. \& KNORRE D. V. 2012. — Rote Liste und gesamtartenliste der Binnenmollusken (Schnecken und Muscheln; Gastropoda et Bivalvia) Deutschlands, in BinOT-HAFKE M., BALZER S., Becker N., Gruttke H., Haupt H., Hofbauer N., Ludwig G., Matzke-Hajek G. \& Strauch M. (éds), Rote Liste der Gefährdeten Tiere, Pflanzen und Pilze Deutschlands. Band 
3: Wirbellose Tiere (Teil 1). Naturschutz und Biologische Vielfalt 70 (3): 647-708.

Kerney M. P., Cameron R. A. D. \& Bertrand A. 2006. - Guide des escargots et limaces d'Europe. Delachaux et Niestlé, Paris, 370 p.

KilleEn I. J., Aldridge D. \& Oliver G. 2004. — Freshwater Bivalves of Britain and Ireland. Field Studies Council, Shrewsbury, 114 p.

Knight A. T., Cowling R. M., Rouget M., Balmford A., Lombard A. T. \& Campbell B. M. 2008. - Knowing but not doing: selecting priority conservation areas and the researchimplementation gap. Conservation Biology 22 (3): 610-617. https://doi.org/10.1111/j.1523-1739.2008.00914.x

Kosmala M., Wiggins A., Swanson A. \& Simmons B. 2016. Assessing data quality in citizen science. Frontiers in Ecology and the Environment 14 (10): 551-560. https://doi.org/10.1002/fee.1436

LAMAND F. \& CUCHERAT X. 2021. - Les Naïades de la région Grand-Est : état des connaissances et perspectives. Naturae 2021 (6): 65-78. https://doi.org/10.5852/naturae2021a6

Larsen B. B., Miller E. C., Rhodes M. K. \& Wiens J. J. 2017. Inordinate fondness multiplied and redistributed: the number of species on earth and the new pie of life. The Quarterly Review of Biology 92 (3): 229-265. https://doi.org/10.1086/693564

Le Berre M., Noble V., Pires M., Médail F. \& Diadema K. 2019. - How to hierarchise species to determine priorities for conservation action? A critical analysis. Biodiversity and Conservation 28 (12): 3051-3071. https://doi.org/10.1007/s10531019-01820-w

LEAKEY R. E. \& LEWIN R. 1996. - The Sixth Extinction: Patterns of Life and the Future of Humankind. Anchor Books, New York, 271 p.

LOCEY K. J. \& LENNON J. T. 2016. - Scaling laws predict global microbial diversity. Proceedings of the National Academy of Sciences 113 (21): 5970-5975. https://doi.org/10.1073/pnas.1521291113

Lydeard C., Cowie R. H., Ponder W. F., Bogan A. E., BouChet P., Clark S. A., Cummings K. S., Frest T. J., Gargominy O., Herbert D. G., Hershler R., Perez K. E., Roth B., Seddon M., Strong E. E. \& Thompson F. G. 2004. - The global decline of nonmarine mollusks. BioScience 54 (4): 321. https://doi.org/10.1641/0006-3568(2004)054[0321:TGDO $\mathrm{NM}] 2.0 . \mathrm{CO} ; 2$

MACE G. M. 2004. - The role of taxonomy in species conservation. Philosophical Transactions of the Royal Society of London. Series B: Biological Sciences 359 (1444): 711-719. https://doi. org/10.1098/rstb.2003.1454

MAMMIDES C. 2019. - European Union's conservation efforts are taxonomically biased. Biodiversity and Conservation 28 (5): 1291 1296. https://doi.org/10.1007/s10531-019-01725-8

MaY R. 1990. — Taxonomy as destiny. Nature 347: 129-130. https://doi.org/10.1038/347129a0

MefFe G. K. \& CARROll C. R. 1997. - Principles of Conservation Biology. Sinauer Associates, Sunderland, Massachusetts, 729 p.

Meyer S., Gargominy O., Haffner P., Poncet L., Robert S. \& TOUROUlT J. 2016. - Outils pour contribuer à la connaissance sur les espèces de l'Inventaire national du Patrimoine naturel. Service du Patrimoine naturel, Muséum national d'Histoire naturel, Paris, 12 p.

Miralles A., Raymond M. \& Lecointre G. 2019. - Empathy and compassion toward other species decrease with evolutionary divergence time. Scientific Reports 9 (19555). https://doi. org/10.1038/s41598-019-56006-9

Mora C., TitTensor D. P., AdL S., Simpson A. G. B. \& Worm B. 2011. - How many species are there on Earth and in the Ocean? PLOS Biology 9 (8): e1001127. https://doi.org/10.1371/journal. pbio. 1001127

Morrison W. R., Lohr J. L., Duchen P., Wilches R., TruJillo D., Mair M. \& RenNer S. S. 2009. - The impact of taxonomic change on conservation: does it kill, can it save, or is it just irrelevant? Biological Conservation 142 (12): 3201-3206. https://doi.org/10.1016/j.biocon.2009.07.019

Pereira H. M., Ferrier S., Walters M., Geller G. N., JonG- man R. H. G., Scholes R. J., Bruford M. W., Brummitt N., Butchart S. H. M., Cardoso A. C., Coops N. C., Dulloo E., Faith D. P., Freyhof J., Gregory R. D., Heip C., Hoft R., Hurtt G., Jetz W., Karp D. S., McGeoch M. A., Obura D., Onoda Y., Pettorelli N., Reyers B., Sayre R., Scharlemann J. P. W., Stuart S. N., Turak E., Walpole M. \& WeGMANn M. 2013. - Essential biodiversity variables. Science 339 (6117): 277-278. https://doi.org/10.1126/science.1229931

Pillon Y. \& Chase M. W. 2007. - Taxonomic exaggeration and its effects on orchid conservation. Conservation Biology 21 (1) 263-265. https://doi.org/10.1111/j.1523-1739.2006.00573.x

Pimm S. L., Jenkins C. N., Abell R., Brooks T. M., GitTleman J. L., Joppa L. N., Raven P. H., Roberts C. M. \& SeXTON J. O. 2014. - The biodiversity of species and their rates of extinction, distribution, and protection. Science 344 (6187): 1246752. https://doi.org/10.1126/science. 1246752

Prié V. 2017. - Nä̈ades et autres bivalves d'eau douce de France. Biotope/MNHN, Paris, 336 p. (Inventaire et Biodiversité).

Prié V., Puillandre N. \& Bouchet P. 2012. — Bad taxonomy can kill: molecular reevaluation of Unio mancus Lamarck, 1819 (Bivalvia: Unionidae) and its accepted subspecies. Knowledge and Management of Aquatic Ecosystems 405 (08), 18 p. https://doi. org $/ 10.1051 / \mathrm{kmae} / 2012014$

Pullin A., Sutherland W., Gardner T., Kapos V. \& Fa J. E. 2013. - Conservation priorities: identifying need, taking action and evaluating success, in MACDONALD D. W. \& WiLlis K. (éds), Key Topics in Conservation Biology 2 [1st ed.]. John Wiley Publishing, Oxford: 2-22. https://doi.org/10.1002/9781118520178.ch1

R CORE TEAM 2020. - R: A Language and Environment for Statistical Computing. Vienna, Austria, R Foundation for Statistical Computing.

Régnier C., Fontaine B. \& Bouchet P. 2009. - Not knowing, not recording, not listing: numerous unnoticed mollusk extinctions. Conservation Biology 23 (5): 1214-1221. https:// doi.org/10.1111/j.1523-1739.2009.01245.x

Régnier C., Achaz G., Lambert A., Cowie R. H., Bouchet P. \& FonTAINE B. 2015. - Mass extinction in poorly known taxa. Proceedings of the National Academy of Sciences of the United States of America 112 (25): 7761-7766. https://doi.org/10.1073/ pnas. 1502350112

Richling I., MalKOwsky Y., KuHn J., NiederhÖFer H.-J. \& Boeters H. D. 2017. - A vanishing hotspot - the impact of molecular insights on the diversity of Central European Bythiospeum Bourguignat, 1882 (Mollusca: Gastropoda: Truncatelloidea). Organisms Diversity \& Evolution 17 (1): 67-85. https:// doi.org/10.1007/s13127-016-0298-y

ROBERGE J.-M. \& ANGELSTAM P. 2004. - Usefulness of the Umbrella species concept as a conservation tool. Conservation Biology 18 (1): 76-85. https://doi.org/10.1111/j.1523-1739.2004.00450.x

Rodríguez J. P., Rojas-SuÁrez F. \& Sharpe C. J. 2004. — Setting priorities for the conservation of Venezuela's threatened birds. Oryx 38 (4): 373-382. https://doi.org/10.1017/S0030605304000730

Rosenberg G. 2014. - A new critical estimate of named specieslevel diversity of the recent Mollusca. American Malacological Bulletin 32 (2): 308. https://doi.org/10.4003/006.032.0204

Rowson B., ANDERSOn R., Turner J. A. \& SyMONDSON W. O. C. 2014. - The slugs of britain and ireland: undetected and undescribed species increase a well-studied, economically important fauna by more than $20 \%$. PLoS ONE 9 (4): e91907. https:// doi.org/10.1371/journal.pone.0091907

Runge C. A., Withey J. C., Naugle D. E., Fargione J. E., Helmstedt K. J., Larsen A. E., MartinuzZi S. \& TACK J. D. 2019. - Single species conservation as an umbrella for management of landscape threats. PLOS ONE 14 (1): e0209619. https:// doi.org/10.1371/journal.pone.0209619

Seddon P. J., Soorae P. S. \& Launay F. 2005. - Taxonomic bias in reintroduction projects. Animal Conservation 8 (1): 51-58. https://doi.org/10.1017/S1367943004001799 
Simberloff D. 1998. — Flagships, umbrellas, and keystones: is single-species management passé in the landscape era? Conservation biology and biodiversity strategies. Biological Conservation 83 (3): 247-257. https://doi.org/10.1016/S0006-3207(97)00081-5

STORK N. E. 2018. - How many species of insects and other terrestrial arthropods are there on earth? Annual Review of Entomology 63 (1): 31-45. https://doi.org/10.1146/annurevento-020117-043348

Strong E. E., Gargominy O., Ponder W. F. \& Bouchet P. 2008. - Global diversity of gastropods (Gastropoda; Mollusca) in freshwater. Hydrobiologia 595: 149-166. https://doi. org/10.1007/s10750-007-9012-6

SysOeV A. \& SCHILEYKO A. 2009. - Land Snails and Slugs of Russia and Adjacent Countries. Pensoft, Sofia Moscow, 312 p. (Pensoft Series Faunistica; 87).

Tessarolo G., Ladle R., Rangel T. \& Hortal J. 2017. — Temporal degradation of data limits biodiversity research. Ecology and Evolution 7 (17): 6863-6870. https://doi.org/10.1002/ece3.3259

Tittensor D. P., Walpole M., Hill S. L. L., Boyce D. G., Britten G. L., Burgess N. D., Butchart S. H. M., Leadley P. W., Regan E. C., Alkemade R., Baumung R., Bellard C., Bouwman L., Bowles-Newark N. J., Chenery A. M., Cheung W.W. L., Christensen V., CoOper H. D., Crowther A. R., Dixon M. J. R., Galli A., Gaveau V., Gregory R. D., Gutierrez N. L., Hirsch T. L., Hoft R., Januchowski-Hartley S. R., Karmann M., KRUG C. B., LeVerINGTON F. J., LOH J., LOJENGA R. K., MALSCH K., MARQUeS A., Morgan D. H. W., Mumby P. J., Newbold T., Noonan-
Mooney K., Pagad S. N., Parks B. C., Pereira H. M., RobERTSON T., RONDININI C., SANTINI L., SCHARLEMANN J. P. W., Schindler S., Sumaila U. R., Teh L. S. L., Van Kolck J., VISCONTI P. \& YE Y. 2014. - A mid-term analysis of progress toward international biodiversity targets. Science 346 (6206) 241-244. https://doi.org/10.1126/science.1257484

Troudet J., Grandcolas P., Blin A., Vignes-Lebbe R. \& LeGENDRE F. 2017. - Taxonomic bias in biodiversity data and societal preferences. Scientific Reports 7 (1): 9132. https://doi. org/10.1038/s41598-017-09084-6

UICN COMITÉ FRANÇAIS, OFB \& MNHN sous presse. — La Liste rouge des espèces menacées en Fance - Chapitre Mollusques continentaux de France métropolitaine. Paris.

Vucetich J., Nelson M. \& Bruskotter J. 2017. — Conservation triage falls short because conservation is not like emergency medicine. Frontiers in Ecology and Evolution 5: 45. https://doi. org/10.3389/fevo.2017.00045

WelteR-SChUlTES F. W. 2012. — European non-marine Molluscs, a Guide for Species Identification. Planet Poster Editions, Göttingen, $679 \mathrm{p}$.

Wilke T., Benke M., BRÄndle M., Albrecht C. \& Bichain J.-M. 2010. - The neglected side of the coin: non-adaptive radiations in spring snails (Bythinella spp.), in Glaubrecht M. (éd.), Evolution in Action. Springer Berlin Heidelberg, Berlin, Heidelberg: 551-578. https://doi.org/10.1007/978-3-642-12425-9_25

WILSON K. A. \& LAW E. A. 2016. - Ethics of conservation triage. Frontiers in Ecology and Evolution 4: 112. https://doi.org/10.3389/ fevo.2016.00112 


\section{ANNEXES}

ANNEXE 1. - Liste des taxons écartés de l'étude.

\section{TAXONS EXCLUS DE LA BASE DE DONNÉES INITIALE}

Anisus septemgyratus (Rossmässler, 1835)

Argna biplicata (Michaud, 1831)

Arion obesoductus P. L. Reischütz, 1973

Bythinella abbreviata (Michaud, 1831)

Bythinella lancelevei Locard, 1884

Bythinella reyniesii (Dupuy, 1851)

Bythiospeum bourguignati (Paladilhe, 1866)

Causa holosericea (S. Studer, 1820)

Cernuella aginnica (Locard, 1894)

Corneola squammatina (Moquin-Tandon, 1855)

Euconulus callopisticus (Bourguignat, 1880)

Hygromia limbata (Draparnaud, 1805)

Omphiscola glabra (O. F. Müller, 1774)

Otala punctata (O. F. Müller, 1774)

Peregriana peregra (O. F. Müller, 1774)

Sphaerium ovale (Férussac, 1807)

Theba pisana (O. F. Müller, 1774)

Truncatella subcylindrica (Linnaeus, 1767)

Viviparus ater (de Cristofori \& Jan, 1832)

Zonitoides arboreus (Say, 1816)

\section{DIFFÉRENCES AVEC LA LISTE DE BICHAIN ET AL. 2019 POUR LA RÉGION GRAND EST}

Hessemilimax kotulae (Westerlund, 1883)

Suppression - étape 2 - niveau de détermination inadéquate

Jaminia quadridens (O. F. Müller, 1774)

Suppression - étape 1 - donnée ancienne $\&$ détermination douteuse

Xeroplexa intersecta (Poiret, 1801)

Suppression - étape 2 - niveau de détermination inadéquate

Unio crassus courtillieri Hattemann, 1859

Suppression - étape 1 - niveau subspécifique

Cernuella aginnica (Locard, 1894)

Suppression - étape 2 - détermination inadéquate

Euconulus praticola (Reinhardt, 1883)

Suppression - étape 1 - considéré comme synonyme d'Euconulus alderi (Gray, 1840) par Horsáková et al. (2020)

Euconulus trochiformis (Montagu, 1803)

Suppression - étape 1 - considéré comme synonyme d'Euconulus fulvus (Müller, 1774) par Horsáková et al. (2020)

Euconulus alderi (Grays, 1840)

Ajout - synonyme plus récent (Horsáková et al. 2020)

Spiralix rayi (Locard, 1882)

Ajout - données bibliographiques

Trochulus clandestinus putonii (Clessin, 1874)

Suppression - étape 1 - niveau subspécifique 
ANNEXE 2. - Liste hiérarchisée des espèces à enjeux de conservation. Les taxons sont présentés par partition taxonomique, par ordre croissant de score global d'enjeux de conservation (SG), par rang dans chacune des partitions et par catégorie de risque. Les notes pour chaque critère sont données ainsi que les valeurs du rapport $\mathrm{M} / \mathrm{H}$ indicatives des moyens déployés pour la conservation. Les actions compensatoires à la conservation (A à $\mathbf{D})$ renvoient au Tableau 3. Abréviations: ab, absent; BFC, Bourgogne-Franche-Comté; Cat, catégorie de risque; GE, Grand-Est; NE, non évalué; pr, présent; R, rareté; RR, responsabilité régionale; $\mathbf{v}$, vulnérabilité.

\begin{tabular}{|c|c|c|c|c|c|c|c|c|c|c|c|c|c|c|}
\hline \multirow[b]{2}{*}{ Groupes } & \multirow[b]{2}{*}{ SG } & \multirow[b]{2}{*}{ Rang } & \multirow[b]{2}{*}{ Cat } & \multicolumn{3}{|c|}{ Critères } & \multicolumn{5}{|c|}{ Actions compensatoires } & \multirow{2}{*}{ Nom valide TAXREF v13.0 } & \multicolumn{2}{|c|}{ Régions } \\
\hline & & & & $\mathbf{R}$ & $\mathbf{V}$ & $\mathbf{R R}$ & $\mathbf{M} / \mathbf{H}$ & A & B & C & D & & BFC & GE \\
\hline \multirow{11}{*}{$\begin{array}{l}\text { Macro- } \\
\text { Bivalves } \\
\text { (Bivalvia) }\end{array}$} & - & - & PoDi & 5 & 5 & 2 & - & - & B1 & - & - & Pseudunio auricularius (Spengler, 1793) & $\mathrm{pr}$ & pr \\
\hline & 26 & 1 & P1 & 3 & 4,33 & 2 & 3 & - & - & - & D1 & Margaritifera margaritifera (Linnaeus, 1758) & pr & pr \\
\hline & 20 & 2 & P2 & 3 & 3,33 & 2 & 1 & - & - & C1 & D1 & Potomida littoralis (Cuvier, 1798) & $\mathrm{pr}$ & pr \\
\hline & 16 & 3 & $\mathrm{P} 2$ & 3 & 2,67 & 2 & 1 & - & - & C1 & D1 & Pseudanodonta complanata (Rossmässler, 1836) & $\mathrm{pr}$ & $\mathrm{pr}$ \\
\hline & 9 & 4 & P3 & 3 & 2 & 1,5 & 1,5 & - & - & C1 & D1 & Unio tumidus Philipsson, 1788 & pr & pr \\
\hline & 8 & 5 & P3 & 4 & 2 & 1 & 0,8 & $\mathrm{~A} 2$ & - & C1 & D1 & Unio mancus Lamarck, 1819 & $\mathrm{pr}$ & pr \\
\hline & 3,67 & 6 & P3 & 1 & 3,67 & 1 & 3 & - & - & - & D1 & Unio crassus Philipsson, 1788 & $\mathrm{pr}$ & pr \\
\hline & 2,67 & 7 & $\mathrm{P} 4$ & 2 & 1,33 & 1 & 1 & - & - & C1 & D1 & Anodonta cygnea (Linnaeus, 1758) & $\mathrm{pr}$ & pr \\
\hline & 1 & 8 & $\mathrm{P} 4$ & 1 & 1 & 1 & 1 & - & - & C1 & D1 & Anodonta anatina (Linnaeus, 1758) & $\mathrm{pr}$ & pr \\
\hline & 1 & 8 & P4 & 1 & 1 & 1 & 1 & - & - & C1 & D1 & Unio pictorum (Linnaeus, 1758) & $\mathrm{pr}$ & pr \\
\hline & - & - & NE & - & - & - & - & - & - & - & - & Sinanodonta woodiana (Lea, 1834) & $\mathrm{pr}$ & pr \\
\hline & - & - & NE & - & - & - & - & - & - & - & - & Corbicula fluminalis (O. F. Müller, 1774) & pr & pr \\
\hline 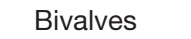 & - & - & NE & - & - & - & - & - & - & - & - & Corbicula leana Prime, 1867 & pr & pr \\
\hline & - & - & NE & - & - & - & - & - & - & - & - & Dreissena polymorpha (Pallas, 1771) & $\mathrm{pr}$ & pr \\
\hline & - & - & NE & - & - & - & - & - & - & - & - & Dreissena bugensis Andrusov, 1897 & $\mathrm{pr}$ & $\mathrm{pr}$ \\
\hline Micro- & 24 & 1 & $\mathrm{P} 2$ & 4 & 3 & 2 & 0,67 & - & - & C1 & D1 & Sphaerium solidum (Normand, 1844) & $\mathrm{pr}$ & $\mathrm{pr}$ \\
\hline Bivalves & 18,67 & 2 & $\mathrm{P} 2$ & 4 & 2,33 & 2 & 0,67 & - & - & C1 & D1 & Sphaerium rivicola (Lamarck, 1818) & $\mathrm{pr}$ & pr \\
\hline (Bivalvia) & 16 & 3 & P2 & 4 & 2 & 2 & 0,75 & A3 & - & C1 & D1 & Euglesa pulchella (Jenyns, 1832) & $\mathrm{pr}$ & pr \\
\hline & 8 & 4 & P3 & 4 & 1 & 2 & 0,5 & A3 & - & C1 & D1 & Euglesa lilljeborgii (Clessin, 1886) & $\mathrm{pr}$ & pr \\
\hline & 8 & 4 & P3 & 4 & 1 & 2 & 0,4 & A3 & $\mathrm{B} 1$ & C1 & - & Odhneripisidium conventus (Clessin, 1877) & $\mathrm{pr}$ & $\mathrm{pr}$ \\
\hline & 7,5 & 5 & P3 & 5 & 1 & 1,5 & 0,33 & $\mathrm{~A} 2$ & B1 & C1 & D1 & Euglesa globularis (Clessin, 1873) & $a b$ & pr \\
\hline & 7,5 & 5 & P3 & 5 & 1 & 1,5 & 0,33 & A3 & B1 & C1 & - & Euglesa pseudosphaerium (J. Favre, 1927) & $a b$ & pr \\
\hline & 4 & 6 & P3 & 4 & 1 & 1 & 0,5 & A3 & - & C1 & D1 & Sphaerium nucleus (S. Studer, 1820) & $\mathrm{pr}$ & pr \\
\hline & 3 & 7 & $\mathrm{P} 4$ & 2 & 1 & 1,5 & 0,5 & A3 & - & C1 & D1 & Euglesa supina (A. Schmidt, 1851) & $\mathrm{pr}$ & pr \\
\hline & 3 & 7 & $\mathrm{P} 4$ & 2 & 1 & 1,5 & 0,5 & A3 & - & C1 & D1 & Odhneripisidium tenuilineatum (Stelfox, 1918) & $\mathrm{pr}$ & pr \\
\hline & 3 & 8 & P4 & 3 & 1 & 1 & 0,5 & A3 & - & C1 & D1 & Euglesa hibernica (Westerlund, 1894) & $\mathrm{pr}$ & pr \\
\hline & 3 & 8 & P4 & 3 & 1 & 1 & 0,75 & A3 & - & C1 & D1 & Euglesa obtusalis (Lamarck, 1818) & $\mathrm{pr}$ & $\mathrm{pr}$ \\
\hline & 2 & 9 & $\mathrm{P} 4$ & 2 & 1 & 1 & 0,75 & A3 & - & C1 & D1 & Euglesa milium (Held, 1836) & $\mathrm{pr}$ & pr \\
\hline & 2 & 9 & $\mathrm{P} 4$ & 2 & 1 & 1 & 0,75 & A3 & - & C1 & D1 & Euglesa personata (Malm, 1855) & $\mathrm{pr}$ & pr \\
\hline & 2 & 9 & P4 & 2 & 1 & 1 & 0,5 & A3 & - & C1 & D1 & Odhneripisidium moitessierianum (Paladilhe, 1866) & $\mathrm{pr}$ & pr \\
\hline & 2 & 9 & $\mathrm{P} 4$ & 2 & 1 & 1 & 0,67 & - & - & C1 & D1 & Pisidium amnicum (O. F. Müller, 1774) & $\mathrm{pr}$ & $\mathrm{pr}$ \\
\hline & 2 & 9 & $\mathrm{P} 4$ & 2 & 1 & 1 & 0,67 & - & - & C1 & D1 & Sphaerium lacustre (O. F. Müller, 1774) & $\mathrm{pr}$ & $\mathrm{pr}$ \\
\hline & 1 & 9 & $\mathrm{P} 4$ & 1 & 1 & 1 & 0,75 & A3 & - & C1 & D1 & sertana (Poli, 1791) & $\mathrm{pr}$ & pr \\
\hline & 1 & 9 & $\mathrm{P} 4$ & 1 & 1 & 1 & 0,75 & A3 & - & C1 & D1 & Euglesa henslowana (Sheppard, 1823) & $\mathrm{pr}$ & pr \\
\hline & 1 & 9 & $\mathrm{P} 4$ & 1 & 1 & 1 & 0,5 & A3 & - & C1 & D1 & Euglesa nitida (Jenyns, 1832) & $\mathrm{pr}$ & pr \\
\hline & 1 & 9 & P4 & 1 & 1 & 1 & 0,5 & A3 & - & C1 & D1 & Euglesa subtruncata (Malm, $1 \varepsilon$ & $\mathrm{pr}$ & pr \\
\hline & 1 & 9 & $\mathrm{P} 4$ & 1 & 1 & 1 & 0,75 & A3 & - & C1 & D1 & Sphaerium corneum (Linnaeus, 1758) & $\mathrm{pr}$ & pr \\
\hline & - & - & NE & NE & NE & NE & - & - & - & - & - & Euglesa compressa (Prime, 1852) & $\mathrm{pr}$ & pr \\
\hline Hydrobies & - & - & PoDi & 5 & 3 & 5 & - & A1 & B1 & C1 & - & Bythinella geisserti Boeters \& Falkner, 2003 & pr & $a b$ \\
\hline (Gastropoda) & - & - & PoDi & 5 & 1 & 5 & - & A1 & B1 & C1 & - & Spiralix burgundina (Locard, 1882) & $\mathrm{pr}$ & $a b$ \\
\hline & 80 & 1 & P1 & 5 & 4 & 4 & 0,83 & A1 & - & - & D1 & Bythinella carinulata (Drouët, 1867) & pr & $a b$ \\
\hline & 75 & 2 & P1 & 5 & 3 & 5 & 2 & - & - & C1 & - & Belgrandia gfrast Haase, 2000 & $a b$ & pr \\
\hline & 75 & 2 & $\mathrm{P} 1$ & 5 & 3 & 5 & 0,2 & $\mathrm{~A} 1$ & $\mathrm{~B} 1$ & C1 & D1 & Bythiospeum drouetianum (Clessin, 1882) & $\mathrm{pr}$ & $a b$ \\
\hline & 75 & 2 & P1 & 5 & 3 & 5 & 0,25 & $\mathrm{~A} 1$ & $\mathrm{~B} 1$ & C1 & - & Islamia spirata (Bernasconi, 1985) & $\mathrm{pr}$ & $a b$ \\
\hline & 64 & 3 & $\mathrm{P} 1$ & 4 & 4 & 4 & 0,71 & $\mathrm{~A} 1$ & $\mathrm{~B} 1$ & - & D1 & Bythinella viridis (Poiret, 1801) & $\mathrm{pr}$ & $\mathrm{pr}$ \\
\hline & 40 & 4 & P1 & 5 & 2 & 4 & 0,22 & A1 & B1 & C1 & - & Bythiospeum diaphanoides Bernasconi, 1985 & $\mathrm{pr}$ & $a b$ \\
\hline & 32 & 5 & $\mathrm{P} 1$ & 4 & 2 & 4 & 0,56 & $\mathrm{~A} 1$ & $\mathrm{~B} 1$ & - & - & Bythiospeum diaphanum (Michaud, 1831) & $\mathrm{pr}$ & $a b$ \\
\hline & 25 & 6 & P1 & 5 & 1 & 5 & 0,33 & $\mathrm{~A} 1$ & - & C1 & D1 & Avenionia bourguignati (Locard, 1883) & $a b$ & $\mathrm{pr}$ \\
\hline & 25 & 6 & P1 & 5 & 1 & 5 & 0,86 & A1 & B1 & C1 & - & Bythinella friderici Boeters \& Falkner, 2008 & $\mathrm{pr}$ & $a b$ \\
\hline & 25 & 6 & P1 & 5 & 1 & 5 & 0,2 & A1 & B1 & C1 & D1 & Bythiospeum moussonianum (Paladilhe, 1869) & $\mathrm{pr}$ & $a b$ \\
\hline & 25 & 6 & $\mathrm{P} 1$ & 5 & 1 & 5 & 0,33 & $\mathrm{~A} 1$ & - & C1 & D1 & Bythiospeum racovitzai (Germain, 1911) & $\mathrm{pr}$ & $a b$ \\
\hline & 25 & 6 & P1 & 5 & 1 & 5 & 0,25 & $\mathrm{~A} 1$ & $\mathrm{~B} 1$ & C1 & D1 & Islamia germaini Boeters \& Falkner, 2003 & $\mathrm{pr}$ & $a b$ \\
\hline & 20 & 7 & $\mathrm{P} 2$ & 5 & 1 & 4 & 0,83 & $\mathrm{~A} 1$ & - & - & D1 & Avenionia brevis (Draparnaud, 1805) & $\mathrm{pr}$ & $a b$ \\
\hline & 20 & 7 & $\mathrm{P} 2$ & 5 & 1 & 4 & 0,56 & A1 & B1 & - & - & Bythiospeum bressanum Bernasconi, 1985 & $\mathrm{pr}$ & $a b$ \\
\hline & 20 & 7 & $\mathrm{P} 2$ & 5 & 1 & 4 & 0,33 & $\mathrm{~A} 1$ & - & C1 & D1 & Bythiospeum francomontanum Bernasconi, 1973 & $\mathrm{pr}$ & $a b$ \\
\hline & 20 & 7 & $\mathrm{P} 2$ & 5 & 1 & 4 & 0,83 & $\mathrm{~A} 1$ & - & - & D1 & Islamia minuta (Draparnaud, 1805) & $\mathrm{pr}$ & $a b$ \\
\hline & 20 & 7 & $\mathrm{P} 2$ & 5 & 1 & 4 & 0,25 & $\mathrm{~A} 1$ & $\mathrm{~B} 1$ & C1 & - & Islamia moquiniana (Dupuy, 1851) & $\mathrm{pr}$ & $a b$ \\
\hline & 20 & 7 & $\mathrm{P} 2$ & 5 & 1 & 4 & 0,5 & A1 & B1 & - & D1 & Spiralix rayi (Locard, 1882) & $\mathrm{pr}$ & pr \\
\hline & 16 & 8 & $\mathrm{P} 2$ & 4 & 1 & 4 & 0,83 & $\mathrm{~A} 1$ & - & - & D1 & Bythinella vesontiana Bernasconi, 1989 & $\mathrm{pr}$ & $a b$ \\
\hline & 16 & 8 & $\mathrm{P} 2$ & 4 & 1 & 4 & 0,22 & $\mathrm{~A} 1$ & $\mathrm{~B} 1$ & C1 & - & Bythiospeum charpyi (Paladilhe, 1867) & $\mathrm{pr}$ & $a b$ \\
\hline & 15 & 9 & $\mathrm{P} 2$ & 5 & 1 & 3 & 0,25 & $\mathrm{~A} 1$ & $\mathrm{~B} 1$ & C1 & D1 & Bythiospeum rhenanum (Lais, 1935) & $a b$ & $\mathrm{pr}$ \\
\hline & 12 & 10 & $\mathrm{P} 2$ & 4 & 1 & 3 & 0,29 & $\mathrm{~A} 1$ & $\mathrm{~B} 1$ & C1 & D1 & Bythinella dunkeri (Frauenfeld, 1857) & $a b$ & pr \\
\hline & - & - & NE & NE & NE & NE & - & - & - & - & - & Emmericia patula (Brumati, 1838) & $\mathrm{pr}$ & $a b$ \\
\hline
\end{tabular}




\begin{tabular}{|c|c|c|c|c|c|c|c|c|c|c|c|c|c|c|}
\hline \multirow[b]{2}{*}{ Groupes } & \multirow[b]{2}{*}{ SG } & \multirow[b]{2}{*}{ Rang } & \multirow[b]{2}{*}{ Cat } & \multicolumn{3}{|c|}{ Critères } & \multicolumn{5}{|c|}{ Actions compensatoires } & \multirow[b]{2}{*}{ Nom valide TAXREF v13.0 } & \multicolumn{2}{|c|}{ Régions } \\
\hline & & & & $\mathbf{R}$ & $\mathbf{v}$ & $\mathbf{R R}$ & $\mathbf{M} / \mathbf{H}$ & A & B & C & D & & BFC & GE \\
\hline & - & - & NE & NE & NE & NE & - & - & - & - & - & Lithoglyphus naticoides (C. Pfeiffer, 1828) & $\mathrm{pr}$ & $\mathrm{pr}$ \\
\hline & - & - & NE & NE & NE & NE & - & - & - & - & - & Potamopyrgus antipodarum (J.E. Gray, 1843) & $\mathrm{pr}$ & $\mathrm{pr}$ \\
\hline Gastéropodes & 23,33 & 1 & P2 & 5 & 2,33 & 2 & 0,86 & A3 & B1 & - & - & Anisus vorticulus (Troschel, 1834) & $a b$ & pr \\
\hline aquatiques & 20 & 2 & $\mathrm{P} 2$ & 5 & 2 & 2 & 0,4 & - & $\mathrm{B} 1$ & C1 & - & Myxas glutinosa (O. F. Müller, 1774) & $\mathrm{pr}$ & $\mathrm{pr}$ \\
\hline (Gastropoda) & 15 & 3 & $\mathrm{P} 2$ & 5 & 1,5 & 2 & 1 & A3 & - & C1 & D1 & Gyraulus rossmaessleri (Auerswald, 1852) & $a b$ & $\mathrm{pr}$ \\
\hline & 12 & 4 & $\mathrm{P} 2$ & 4 & 1,5 & 2 & 2,5 & - & - & C1 & - & Viviparus contectus (Millet, 1813) & $a b$ & $\mathrm{pr}$ \\
\hline & 10 & 5 & P3 & 5 & 1 & 2 & 1,67 & - & - & C1 & - & Valvata macrostoma Mörch, 1864 & $\mathrm{pr}$ & $\mathrm{pr}$ \\
\hline & 8 & 6 & P3 & 4 & 1 & 2 & 1 & A3 & - & C1 & D1 & Gyraulus laevis (Alder, 1838) & pr & $\mathrm{pr}$ \\
\hline & 7,5 & 7 & P3 & 5 & 1 & 1,5 & 0,4 & $\mathrm{~A} 2$ & - & C1 & - & Ampullaceana ampla (W. Hartmann, 1821) & $\mathrm{pr}$ & $\mathrm{pr}$ \\
\hline & 6 & 8 & P3 & 4 & 1,5 & 1 & 1 & A3 & - & C1 & D1 & Anisus spirorbis (Linnaeus, 1758) & $\mathrm{pr}$ & $\mathrm{pr}$ \\
\hline & 6 & 8 & P3 & 4 & 1,5 & 1 & 2,5 & - & - & C1 & - & Bithynia leachii (Sheppard, 1823) & $\mathrm{pr}$ & $\mathrm{pr}$ \\
\hline & 5 & 9 & P3 & 5 & 1 & 1 & 0,5 & $\mathrm{~A} 2$ & B1 & C1 & D1 & Stagnicola corvus (Gmelin, 1791) & $a b$ & $\mathrm{pr}$ \\
\hline & 4,5 & 10 & P3 & 3 & 1,5 & 1 & 2 & - & - & C1 & D1 & Aplexa hypnorum (Linnaeus, 1758) & $\mathrm{pr}$ & $\mathrm{pr}$ \\
\hline & 4,5 & 10 & P3 & 3 & 1,5 & 1 & 1,5 & - & - & C1 & D1 & Physa fontinalis (Linnaeus, 1758) & $\mathrm{pr}$ & pr \\
\hline & 4 & 11 & P3 & 4 & 1 & 1 & 1,5 & - & - & C1 & D1 & Segmentina nitida (O. F. Müller, 1774) & $\mathrm{pr}$ & $\mathrm{pr}$ \\
\hline & 4 & 11 & P3 & 4 & 1 & 1 & 0,6 & $\mathrm{~A} 2$ & - & C1 & D1 & Stagnicola fuscus (C. Pfeiffer, 1821) & $\mathrm{pr}$ & pr \\
\hline & 4 & 11 & P3 & 4 & 1 & 1 & 0,8 & $\mathrm{~A} 2$ & - & C1 & D1 & Stagnicola palustris (O. F. Müller, 1774) & $\mathrm{pr}$ & pr \\
\hline & 3 & 12 & P4 & 3 & 1 & 1 & 1,25 & A3 & - & C1 & - & Anisus leucostoma (Millet, 1813) & $\mathrm{pr}$ & $\mathrm{pr}$ \\
\hline & 3 & 12 & P4 & 3 & 1 & 1 & 0,75 & A3 & - & C1 & D1 & Anisus vortex (Linnaeus, 1758) & $\mathrm{pr}$ & $\mathrm{pr}$ \\
\hline & 3 & 12 & P4 & 3 & 1 & 1 & 1 & A3 & - & C1 & D1 & Amiger crista (Linnaeus, 1758) & $\mathrm{pr}$ & pr \\
\hline & 3 & 12 & P4 & 3 & 1 & 1 & 0,75 & A3 & - & C1 & D1 & Peregriana labiata (Rossmässler, 1835) & $\mathrm{pr}$ & $a b$ \\
\hline & 3 & 12 & P4 & 3 & 1 & 1 & 1,33 & - & - & C1 & D1 & Planorbis planorbis (Linnaeus, 1758) & $\mathrm{pr}$ & pr \\
\hline & 3 & 12 & P4 & 3 & 1 & 1 & 1,5 & - & - & $\mathrm{C} 1$ & D1 & Theodoxus fluviatilis (Linnaeus, 1758) & $\mathrm{pr}$ & $\mathrm{pr}$ \\
\hline & 2 & 13 & P4 & 2 & 1 & 1 & 1,5 & - & - & C1 & D1 & Planorbarius corneus (Linnaeus, 1758) & $\mathrm{pr}$ & $\mathrm{pr}$ \\
\hline & 2 & 14 & P4 & 2 & 1 & 1 & 1,5 & - & - & C1 & D1 & Acroloxus lacustris (Linnaeus, 1758) & $\mathrm{pr}$ & pr \\
\hline & 2 & 14 & P4 & 2 & 1 & 1 & 0,6 & $\mathrm{~A} 2$ & - & C1 & D1 & Ampullaceana balthica (Linnaeus, 1758) & $\mathrm{pr}$ & pr \\
\hline & 2 & 14 & P4 & 2 & 1 & 1 & 2 & - & - & C1 & D1 & Bathyomphalus contortus (Linnaeus, 1758) & $\mathrm{pr}$ & pr \\
\hline & 2 & 14 & P4 & 2 & 1 & 1 & 2 & - & - & C1 & D1 & Hippeutis complanatus (Linnaeus, 1758) & $\mathrm{pr}$ & $\mathrm{pr}$ \\
\hline & 2 & 14 & P4 & 2 & 1 & 1 & 1,5 & - & - & C1 & D1 & Lymnaea stagnalis (Linnaeus, 1758) & $\mathrm{pr}$ & pr \\
\hline & 2 & 14 & P4 & 2 & 1 & 1 & 1 & - & - & C1 & D1 & Planorbis carinatus O. F. Müller, 1774 & $\mathrm{pr}$ & pr \\
\hline & 2 & 14 & P4 & 2 & 1 & 1 & 0,6 & $\mathrm{~A} 2$ & - & C1 & D1 & Radix auricularia (Linnaeus, 1758) & $\mathrm{pr}$ & $\mathrm{pr}$ \\
\hline & 2 & 14 & P4 & 2 & 1 & 1 & 1,33 & - & - & C1 & D1 & Valvata cristata O. F. Müller, 1774 & $\mathrm{pr}$ & pr \\
\hline & 2 & 14 & P4 & 2 & 1 & 1 & 1,5 & - & - & C1 & D1 & Viviparus viviparus (Linnaeus, 1758) & $\mathrm{pr}$ & $\mathrm{pr}$ \\
\hline & 1 & 15 & P4 & 1 & 1 & 1 & 1,5 & - & - & C1 & D1 & Ancylus fluviatilis O. F. Müller, 1774 & $\mathrm{pr}$ & pr \\
\hline & 1 & 15 & P4 & 1 & 1 & 1 & 1,5 & - & - & C1 & D1 & Bithynia tentaculata (Linnaeus, 1758) & $\mathrm{pr}$ & pr \\
\hline & 1 & 15 & P4 & 1 & 1 & 1 & 1,5 & - & - & C1 & D1 & Galba truncatula (O. F. Müller, 1774) & $\mathrm{pr}$ & pr \\
\hline & 1 & 15 & P4 & 1 & 1 & 1 & 0,75 & A3 & - & C1 & D1 & Gyraulus albus (O. F. Müller, 1774) & $\mathrm{pr}$ & $\mathrm{pr}$ \\
\hline & 1 & 15 & P4 & 1 & 1 & 1 & 1 & - & - & C1 & D1 & Valvata piscinalis (O. F. Müller, 1774) & $\mathrm{pr}$ & pr \\
\hline & - & - & NE & NE & NE & NE & - & - & - & - & - & Ferrissia californica (Rowel, 1863) & $\mathrm{pr}$ & pr \\
\hline & - & - & NE & NE & NE & NE & - & - & - & - & - & Gyraulus parvus (Say, 1817) & $\mathrm{pr}$ & $\mathrm{pr}$ \\
\hline & - & - & NE & NE & NE & NE & - & - & - & - & - & Menetus dilatatus (Gould, 1841) & $\mathrm{pr}$ & $\mathrm{pr}$ \\
\hline & - & - & NE & NE & NE & NE & - & - & - & - & - & Physella acuta (Draparnaud, 1805) & $\mathrm{pr}$ & $\mathrm{pr}$ \\
\hline Limaces & - & - & PoDi & 5 & 1 & 1,5 & - & A2 & B1 & C1 & - & Deroceras rodnae Grossu \& Lupu, 1965 & $a b$ & pr \\
\hline & 6 & 1 & P3 & 4 & 1 & 1,5 & 2 & - & - & C1 & D1 & Tandonia rustica (Millet, 1843) & $\mathrm{pr}$ & pr \\
\hline & 5 & 2 & P3 & 5 & 1 & 1 & 0,4 & $\mathrm{~A} 2$ & - & C1 & D1 & Arion hortensis A. Férussac, 1819 & pr & pr \\
\hline & 5 & 2 & P3 & 5 & 1 & 1 & 0,8 & $\mathrm{~A} 2$ & - & C1 & D1 & Deroceras agreste (Linnaeus, 1758) & $\mathrm{pr}$ & pr \\
\hline & 4 & 3 & P3 & 4 & 1 & 1 & 0,4 & $\mathrm{~A} 2$ & - & C1 & D1 & Arion distinctus J. Mabille, 1868 & $\mathrm{pr}$ & pr \\
\hline & 4 & 3 & P3 & 4 & 1 & 1 & 0,4 & $\mathrm{~A} 2$ & - & C1 & D1 & Arion fasciatus (Nilsson, 1823) & $a b$ & pr \\
\hline & 4 & 3 & P3 & 4 & 1 & 1 & 0,4 & $\mathrm{~A} 2$ & - & C1 & D1 & Arion fuscus (O. F. Müller, 1774) & $\mathrm{pr}$ & pr \\
\hline & 4 & 3 & P3 & 4 & 1 & 1 & 0,6 & $\mathrm{~A} 2$ & - & C1 & D1 & Arion subfuscus (Draparnaud, 1805) & $\mathrm{pr}$ & $\mathrm{pr}$ \\
\hline & 3 & 4 & P4 & 3 & 1 & 1 & 0,6 & $\mathrm{~A} 2$ & - & C1 & D1 & Arion rufus (Linnaeus, 1758) & $\mathrm{pr}$ & $\mathrm{pr}$ \\
\hline & 3 & 4 & P4 & 3 & 1 & 1 & 0,6 & $\mathrm{~A} 2$ & - & C1 & D1 & Deroceras laeve (O. F. Müller, 1774) & $\mathrm{pr}$ & $\mathrm{pr}$ \\
\hline & 3 & 4 & P4 & 3 & 1 & 1 & 1,5 & - & - & C1 & D1 & Lehmannia marginata (O. F. Müller, 1774) & $\mathrm{pr}$ & $\mathrm{pr}$ \\
\hline & 3 & 4 & P4 & 3 & 1 & 1 & 1,5 & - & - & C1 & D1 & Malacolimax tenellus (O. F. Müller, 1774) & $\mathrm{pr}$ & pr \\
\hline & 2 & 5 & P4 & 2 & 1 & 1 & 1 & - & - & C1 & D1 & Limax cinereoniger Wolf, 1803 & $\mathrm{pr}$ & $\mathrm{pr}$ \\
\hline & 2 & 5 & P4 & 2 & 1 & 1 & 1,5 & - & - & C1 & D1 & Limax maximus Linnaeus, 1758 & $\mathrm{pr}$ & $\mathrm{pr}$ \\
\hline & - & - & NE & NE & NE & NE & - & - & - & - & - & Ambigolimax valentianus (A. Férussac, 1822) & $a b$ & $\mathrm{pr}$ \\
\hline & - & - & NE & NE & NE & NE & - & - & - & - & - & Arion intermedius Normand, 1852 & $\mathrm{pr}$ & pr \\
\hline & - & - & NE & NE & NE & NE & - & - & - & - & - & Arion vulgaris Moquin-Tandon, 1855 & $\mathrm{pr}$ & $\mathrm{pr}$ \\
\hline & - & - & NE & NE & NE & NE & - & - & - & - & - & Boettgerilla pallens Simroth, 1912 & $\mathrm{pr}$ & $\mathrm{pr}$ \\
\hline & - & - & NE & NE & NE & $\mathrm{NE}$ & - & - & - & - & - & $\begin{array}{l}\text { Deroceras invadens Reise, Hutchinson, } \\
\text { Schunack \& Schlitt, } 2011\end{array}$ & $\mathrm{pr}$ & $\mathrm{pr}$ \\
\hline & - & - & NE & NE & NE & NE & - & - & - & - & - & Deroceras klemmi Grossu, 1972 & $a b$ & pr \\
\hline & - & - & NE & NE & NE & NE & - & - & - & - & - & Deroceras reticulatum (O. F. Müller, 1774) & $\mathrm{pr}$ & $\mathrm{pr}$ \\
\hline & - & - & NE & NE & NE & NE & - & - & - & - & - & Limacus flavus (Linnaeus, 1758) & $\mathrm{pr}$ & $\mathrm{pr}$ \\
\hline & - & - & NE & NE & NE & NE & - & - & - & - & - & Milax gagates (Draparnaud, 1801) & $a b$ & $\mathrm{pr}$ \\
\hline
\end{tabular}




\begin{tabular}{|c|c|c|c|c|c|c|c|c|c|c|c|c|c|c|}
\hline \multirow[b]{2}{*}{ Groupes } & \multirow[b]{2}{*}{ SG } & \multirow[b]{2}{*}{ Rang } & \multirow[b]{2}{*}{ Cat } & \multicolumn{3}{|c|}{ Critères } & \multicolumn{5}{|c|}{ Actions compensatoires } & \multirow[b]{2}{*}{ Nom valide TAXREF v13.0 } & Régi & ions \\
\hline & & & & $\mathbf{R}$ & $\mathbf{v}$ & $\mathbf{R R}$ & $\mathbf{M} / \mathbf{H}$ & $\mathbf{A}$ & B & C & D & & BFC & GE \\
\hline Escargots & - & - & PoDi & 5 & 1 & 1,5 & - & - & B1 & C1 & - & Acicula fusca (Montagu, 1803) & $\mathrm{pr}$ & $a b$ \\
\hline (Gastropoda) & - & - & PoDi & 5 & 1,5 & 2 & - & $\mathrm{A} 2$ & B1 & C1 & - & Helicopsis striata (O. F. Müller, 1774) & $a b$ & $\mathrm{pr}$ \\
\hline & - & - & PoDi & 5 & 1 & 1,5 & - & - & B1 & $\mathrm{C} 1$ & - & Perforatella bidentata (Gmelin, 1791) & $a b$ & $\mathrm{pr}$ \\
\hline & - & - & PoDi & 5 & 2,5 & 2 & - & - & B1 & C1 & - & Vallonia declivis Sterki, 1893 & $a b$ & $\mathrm{pr}$ \\
\hline & 80 & 1 & P1 & 5 & 4 & 4 & 0,86 & A1 & $\mathrm{B} 1$ & - & D1 & Truncatellina arcyensis Klemm, 1943 & $\mathrm{pr}$ & $a b$ \\
\hline & 15 & 2 & $\mathrm{P} 2$ & 5 & 1,5 & 2 & 2 & - & - & - & D1 & Vertigo genesii (Gredler, 1856) & $\mathrm{pr}$ & $a b$ \\
\hline & 15 & 2 & $\mathrm{P} 2$ & 5 & 1,5 & 2 & 0,4 & $\mathrm{~A} 2$ & - & C1 & D1 & Xerocrassa geyeri (Soós, 1926) & $\mathrm{pr}$ & $\mathrm{pr}$ \\
\hline & 12 & 3 & $\mathrm{P} 2$ & 3 & 1 & 4 & 1,5 & - & - & C1 & D1 & Obscurella conica (Vallot, 1801) & $\mathrm{pr}$ & pr \\
\hline & 12 & 3 & P2 & 4 & 1 & 3 & 1,33 & - & - & $\mathrm{C} 1$ & D1 & Platyla dupuyi (Paladilhe, 1868) & $\mathrm{pr}$ & $\mathrm{pr}$ \\
\hline & 11,25 & 4 & P3 & 5 & 1,5 & 1,5 & 0,67 & - & - & C1 & D1 & Columella columella (G. von Martens, 1830) & $\mathrm{pr}$ & $a b$ \\
\hline & 11,25 & 4 & P3 & 5 & 1,5 & 1,5 & 1,25 & A3 & - & C1 & - & Nesovitrea petronella (L. Pfeiffer, 1853) & $\mathrm{pr}$ & $\mathrm{pr}$ \\
\hline & 11,25 & 4 & P3 & 5 & 1,5 & 1,5 & 0,67 & - & - & C1 & D1 & Pupilla alpicola (Charpentier, 1837) & $\mathrm{pr}$ & $\mathrm{pr}$ \\
\hline & 10,67 & 5 & P3 & 4 & 1,33 & 2 & 1,33 & - & - & C1 & D1 & Vallonia enniensis (Gredler, 1856) & $\mathrm{pr}$ & $\mathrm{pr}$ \\
\hline & 10 & 6 & P3 & 5 & 1 & 2 & 1 & - & - & C1 & D1 & Cochlodina orthostoma (Menke, 1828) & $\mathrm{pr}$ & $a b$ \\
\hline & 10 & 6 & P3 & 5 & 1 & 2 & 1,2 & A3 & B1 & C1 & - & Hessemilimax kotulae (Westerlund, 1883) & $\mathrm{pr}$ & $a b$ \\
\hline & 10 & 6 & P3 & 5 & 1 & 2 & 0,4 & $\mathrm{~A} 2$ & - & C1 & D1 & Quickella arenaria (Potiez \& Michaud, 1838) & $\mathrm{pr}$ & $a b$ \\
\hline & 10 & 6 & P3 & 5 & 1 & 2 & 0,29 & $\mathrm{~A} 2$ & B1 & C1 & D1 & Semilimax semilimax (J. Férussac, 1802) & $a b$ & $\mathrm{pr}$ \\
\hline & 10 & 6 & P3 & 5 & 1 & 2 & 0,25 & $\mathrm{~A} 1$ & B1 & C1 & D1 & Trochulus clandestinus (W. Hartmann, 1821) & $a b$ & $\mathrm{pr}$ \\
\hline & 10 & 6 & P3 & 5 & 1 & 2 & 0,5 & A3 & - & C1 & D1 & Vitrinobrachium breve (A. Férussac, 1821) & $a b$ & $\mathrm{pr}$ \\
\hline & 8 & 7 & P3 & 3 & 1,33 & 2 & 1,5 & - & - & C1 & D1 & Chondrula tridens (O. F. Müller, 1774) & $\mathrm{pr}$ & $\mathrm{pr}$ \\
\hline & 8 & 7 & P3 & 4 & 1 & 2 & 0,67 & - & - & C1 & D1 & Clausilia corynodes Held, 1836 & $\mathrm{pr}$ & $\mathrm{pr}$ \\
\hline & 8 & 7 & P3 & 4 & 1 & 2 & 0,5 & A3 & - & C1 & D1 & Daudebardia brevipes (Draparnaud, 1805) & $a b$ & $\mathrm{pr}$ \\
\hline & 8 & 7 & P3 & 4 & 1 & 2 & 0,5 & A3 & - & C1 & D1 & Daudebardia rufa (Draparnaud, 1805) & $a b$ & $\mathrm{pr}$ \\
\hline & 8 & 7 & P3 & 4 & 1 & 2 & 1 & - & - & C1 & D1 & Pagodulina pagodula (Des Moulins, 1830) & $\mathrm{pr}$ & $\mathrm{pr}$ \\
\hline & 7,5 & 8 & P3 & 5 & 1 & 1,5 & 0,29 & $\mathrm{~A} 2$ & B1 & $\mathrm{C} 1$ & D1 & Aegopinella minor (Stabile, 1864) & $a b$ & $\mathrm{pr}$ \\
\hline & 7,5 & 8 & P3 & 5 & 1 & 1,5 & 0,67 & - & - & C1 & D1 & Alinda biplicata (Montagu, 1803) & $a b$ & $\mathrm{pr}$ \\
\hline & 7,5 & 8 & P3 & 5 & 1 & 1,5 & 1,33 & - & - & C1 & D1 & Discus ruderatus (W. Hartmann, 1821) & $a b$ & $\mathrm{pr}$ \\
\hline & 7,5 & 8 & P3 & 5 & 1 & 1,5 & 0,67 & - & - & C1 & D1 & Granaria variabilis (Draparnaud, 1801) & $\mathrm{pr}$ & $a b$ \\
\hline & 7,5 & 8 & P3 & 5 & 1 & 1,5 & 0,5 & A3 & - & C1 & D1 & Morlina glabra (Rossmässler, 1835) & $\mathrm{pr}$ & pr \\
\hline & 7,5 & 8 & P3 & 5 & 1 & 1,5 & 0,67 & - & - & C1 & D1 & Pupilla sterrii (Voith, 1840) & $\mathrm{pr}$ & $a b$ \\
\hline & 7,5 & 8 & P3 & 5 & 1 & 1,5 & 0,5 & $\mathrm{~A} 1$ & - & C1 & D1 & Trochulus striolatus (C. Pfeiffer, 1828) & $\mathrm{pr}$ & $\mathrm{pr}$ \\
\hline & 7,5 & 8 & P3 & 5 & 1 & 1,5 & 0,5 & - & B1 & C1 & - & Truncatellina claustralis (Gredler, 1856) & $\mathrm{pr}$ & $a b$ \\
\hline & 7,5 & 8 & P3 & 5 & 1 & 1,5 & 0,67 & - & B1 & C1 & - & Zonitoides excavatus (Alder, 1830) & $a b$ & $\mathrm{pr}$ \\
\hline & 7,5 & 9 & P3 & 5 & 1,5 & 1 & 1 & $\mathrm{~A} 2$ & B1 & C1 & - & Cochlicopa nitens (M. von Gallenstein, 1848) & $a b$ & pr \\
\hline & 7 & 10 & P3 & 3 & 2,33 & 1 & 1,67 & - & - & - & D1 & Vertigo angustior Jeffreys, 1830 & $\mathrm{pr}$ & $\mathrm{pr}$ \\
\hline & 6 & 11 & P3 & 3 & 1 & 2 & 1 & - & - & C1 & D1 & Laciniaria plicata (Draparnaud, 1801) & $\mathrm{pr}$ & $\mathrm{pr}$ \\
\hline & 6 & 12 & P3 & 3 & 1 & 2 & 2 & - & - & - & D1 & Vertigo geyeri Lindholm, 1925 & $\mathrm{pr}$ & $a b$ \\
\hline & 6 & 13 & P3 & 4 & 1 & 1,5 & 1,5 & - & - & C1 & D1 & Azeca goodalli (A. Férussac, 1821) & $\mathrm{pr}$ & pr \\
\hline & 6 & 13 & P3 & 4 & 1 & 1,5 & 1 & - & - & C1 & D1 & Cochlodina fimbriata (Rossmässler, 1835) & $\mathrm{pr}$ & $\mathrm{pr}$ \\
\hline & 6 & 13 & P3 & 4 & 1 & 1,5 & 0,67 & - & - & C1 & D1 & Granaria frumentum (Draparnaud, 1801) & $\mathrm{pr}$ & $\mathrm{pr}$ \\
\hline & 6 & 13 & P3 & 4 & 1 & 1,5 & 0,67 & - & - & C1 & D1 & Granopupa granum (Draparnaud, 1801) & $\mathrm{pr}$ & $a b$ \\
\hline & 6 & 13 & P3 & 4 & 1 & 1,5 & 2 & - & - & C1 & D1 & Jaminia quadridens (O. F. Müller, 1774) & $\mathrm{pr}$ & $a b$ \\
\hline & 6 & 13 & P3 & 4 & 1 & 1,5 & 0,6 & $\mathrm{~A} 2$ & - & C1 & D1 & Oxyloma sarsii (Esmark, 1886) & $\mathrm{pr}$ & $\mathrm{pr}$ \\
\hline & 6 & 13 & P3 & 4 & 1 & 1,5 & 0,67 & - & - & C1 & D1 & Platyla polita (W. Hartmann, 1840) & $\mathrm{pr}$ & $\mathrm{pr}$ \\
\hline & 6 & 13 & P3 & 4 & 1 & 1,5 & 0,67 & $\mathrm{~A} 1$ & - & C1 & D1 & Trochulus montanus (S. Studer, 1820) & $\mathrm{pr}$ & $\mathrm{pr}$ \\
\hline & 6 & 13 & P3 & 4 & 1 & 1,5 & 1,33 & - & - & C1 & D1 & Vertigo alpestris Alder, 1838 & $\mathrm{pr}$ & $\mathrm{pr}$ \\
\hline & 6 & 13 & P3 & 4 & 1 & 1,5 & 1 & - & - & C1 & D1 & Vitrea diaphana (S. Studer, 1820) & $\mathrm{pr}$ & $\mathrm{pr}$ \\
\hline & 6 & 14 & P3 & 2 & 3 & 1 & 1,67 & - & - & - & D1 & Vertigo moulinsiana (Dupuy, 1849) & $\mathrm{pr}$ & $\mathrm{pr}$ \\
\hline & 4,5 & 15 & P3 & 3 & 1 & 1,5 & 1 & - & - & C1 & D1 & Clausilia cruciata (S. Studer, 1820) & $\mathrm{pr}$ & $\mathrm{pr}$ \\
\hline & 4,5 & 15 & P3 & 3 & 1 & 1,5 & 1 & - & - & C1 & D1 & Clausilia dubia Draparnaud, 1805 & $\mathrm{pr}$ & pr \\
\hline & 4,5 & 15 & P3 & 3 & 1 & 1,5 & 1,5 & - & - & C1 & D1 & Edentiella edentula (Draparnaud, 1805) & $\mathrm{pr}$ & $\mathrm{pr}$ \\
\hline & 4,5 & 15 & P3 & 3 & 1 & 1,5 & 1 & A3 & - & C1 & D1 & Eucobresia diaphana (Draparnaud, 1805) & $\mathrm{pr}$ & $\mathrm{pr}$ \\
\hline & 4,5 & 15 & P3 & 3 & 1 & 1,5 & 1,5 & - & - & C1 & D1 & Macularia sylvatica (Draparnaud, 1801) & $\mathrm{pr}$ & $a b$ \\
\hline & 4,5 & 15 & P3 & 3 & 1 & 1,5 & 1,5 & - & - & C1 & D1 & Orcula dolium (Draparnaud, 1801) & $\mathrm{pr}$ & $\mathrm{pr}$ \\
\hline & 4,5 & 15 & P3 & 3 & 1 & 1,5 & 0,75 & A3 & - & C1 & D1 & Oxychilus navarricus (Bourguignat, 1870) & $\mathrm{pr}$ & $\mathrm{pr}$ \\
\hline & 4,5 & 15 & P3 & 3 & 1 & 1,5 & 1 & A3 & - & C1 & D1 & Pseudotrichia rubiginosa (Rossmässler, 1838) & $\mathrm{pr}$ & pr \\
\hline & 4,5 & 15 & P3 & 3 & 1 & 1,5 & 1 & - & - & C1 & D1 & Pupilla triplicata (S. Studer, 1820) & $\mathrm{pr}$ & $\mathrm{pr}$ \\
\hline & 4,5 & 15 & P3 & 3 & 1 & 1,5 & 0,5 & $\mathrm{~A} 1$ & - & C1 & D1 & Trochulus villosus (Draparnaud, 1805) & $\mathrm{pr}$ & $\mathrm{pr}$ \\
\hline & 4,5 & 15 & P3 & 3 & 1 & 1,5 & 1,33 & - & - & C1 & - & Vertigo substriata (Jeffreys, 1833) & $\mathrm{pr}$ & $\mathrm{pr}$ \\
\hline & 4 & 16 & P3 & 4 & 1 & 1 & 1 & - & - & C1 & D1 & Columella aspera Waldén, 1966 & $\mathrm{pr}$ & $\mathrm{pr}$ \\
\hline & 4 & 16 & P3 & 4 & 1 & 1 & 1,5 & - & - & C1 & D1 & Euomphalia strigella (Draparnaud, 1801) & $\mathrm{pr}$ & $\mathrm{pr}$ \\
\hline & 3 & 17 & P4 & 2 & 1 & 1,5 & 1 & - & - & C1 & D1 & Macrogastra attenuata (Rossmässler, 1835) & $\mathrm{pr}$ & $\mathrm{pr}$ \\
\hline & 3 & 17 & P4 & 2 & 1 & 1,5 & 1 & - & - & C1 & D1 & Macrogastra plicatula (Draparnaud, 1801) & $\mathrm{pr}$ & $\mathrm{pr}$ \\
\hline & 3 & 17 & P4 & 2 & 1 & 1,5 & 1 & - & - & C1 & D1 & Macrogastra ventricosa (Draparnaud, 1801) & $\mathrm{pr}$ & pr \\
\hline & 3 & 17 & P4 & 2 & 1 & 1,5 & 1 & - & - & C1 & D1 & Vitrea subrimata (Reinhardt, 1871) & $\mathrm{pr}$ & $\mathrm{pr}$ \\
\hline & 3 & 18 & P4 & 3 & 1 & 1 & 1 & - & - & C1 & D1 & Acicula lineata (Draparnaud, 1801) & $\mathrm{pr}$ & $\mathrm{pr}$ \\
\hline & 3 & 18 & P4 & 3 & 1 & 1 & 1 & - & - & C1 & D1 & Balea perversa (Linnaeus, 1758) & $\mathrm{pr}$ & $\mathrm{pr}$ \\
\hline & 3 & 18 & P4 & 3 & 1 & 1 & 0,6 & $\mathrm{~A} 2$ & - & C1 & D1 & Cochlicopa lubricella (Porro, 1838) & $\mathrm{pr}$ & $\mathrm{pr}$ \\
\hline
\end{tabular}




\begin{tabular}{|c|c|c|c|c|c|c|c|c|c|c|c|c|c|c|}
\hline \multirow[b]{2}{*}{ Groupes } & \multirow[b]{2}{*}{ SG } & \multirow[b]{2}{*}{ Rang } & \multirow[b]{2}{*}{ Cat } & \multicolumn{3}{|c|}{ Critères } & \multicolumn{5}{|c|}{ Actions compensatoires } & \multirow[b]{2}{*}{ Nom valide TAXREF v13.0 } & \multicolumn{2}{|c|}{ Régions } \\
\hline & & & & $\mathbf{R}$ & $\mathbf{v}$ & $\mathbf{R R}$ & $\mathbf{M} / \mathbf{H}$ & A & B & C & D & & BFC & GE \\
\hline & 3 & 18 & $\mathrm{P} 4$ & 3 & 1 & 1 & 1 & - & - & C1 & D1 & Macrogastra rolphii (Turton, 1826) & pr & pr \\
\hline & 3 & 18 & $\mathrm{P} 4$ & 3 & 1 & 1 & 0,5 & A3 & - & C1 & D1 & Oxychilus alliarius (J. S. Miller, 1822) & $\mathrm{pr}$ & $\mathrm{pr}$ \\
\hline & 3 & 18 & $\mathrm{P} 4$ & 3 & 1 & 1 & 0,75 & A3 & - & C1 & D1 & Oxychilus draparnaudi (H. Beck, 1837) & $\mathrm{pr}$ & $\mathrm{pr}$ \\
\hline & 3 & 18 & $\mathrm{P} 4$ & 3 & 1 & 1 & 1 & - & - & C1 & D1 & Sphyradium doliolum (Bruguière, 1792) & $\mathrm{pr}$ & pr \\
\hline & 3 & 18 & $\mathrm{P} 4$ & 3 & 1 & 1 & 0,6 & $\mathrm{~A} 2$ & - & C1 & D1 & Succinella oblonga (Draparnaud, 1801) & $\mathrm{pr}$ & $\mathrm{pr}$ \\
\hline & 3 & 18 & $\mathrm{P} 4$ & 3 & 1 & 1 & 0,33 & A1 & - & C1 & D1 & Trochulus sericeus (Draparnaud, 1801) & $\mathrm{pr}$ & $\mathrm{pr}$ \\
\hline & 3 & 18 & $\mathrm{P} 4$ & 3 & 1 & 1 & 1 & - & - & C1 & D1 & Truncatellina callicratis (Scacchi, 1833) & $\mathrm{pr}$ & $\mathrm{pr}$ \\
\hline & 3 & 18 & $\mathrm{P} 4$ & 3 & 1 & 1 & 1 & - & - & C1 & D1 & Zebrina detrita (O. F. Müller, 1774) & $\mathrm{pr}$ & pr \\
\hline & 2 & 19 & $\mathrm{P} 4$ & 1 & 2 & 1 & 0,5 & A3 & - & $\mathrm{C} 1$ & D1 & Phenacolimax major (A. Férussac, 1807) & $\mathrm{pr}$ & pr \\
\hline & 2 & 20 & $\mathrm{P} 4$ & 2 & 1 & 1 & 0,5 & A3 & - & C1 & D1 & Aegopinella nitidula (Draparnaud, 1805) & $\mathrm{pr}$ & $\mathrm{pr}$ \\
\hline & 2 & 20 & P4 & 2 & 1 & 1 & 0,75 & A3 & - & C1 & D1 & Candidula unifasciata (Poiret, 1801) & $\mathrm{pr}$ & $\mathrm{pr}$ \\
\hline & 2 & 20 & P4 & 2 & 1 & 1 & 1 & - & - & C1 & D1 & Chondrina avenacea (Bruguière, 1792) & $\mathrm{pr}$ & pr \\
\hline & 2 & 20 & $\mathrm{P} 4$ & 2 & 1 & 1 & 1 & - & - & C1 & D1 & Columella edentula (Draparnaud, 1805) & $\mathrm{pr}$ & $\mathrm{pr}$ \\
\hline & 2 & 20 & P4 & 2 & 1 & 1 & 1,5 & - & - & C1 & D1 & Ena montana (Draparnaud, 1801) & $\mathrm{pr}$ & $\mathrm{pr}$ \\
\hline & 2 & 20 & $\mathrm{P} 4$ & 2 & 1 & 1 & 1,33 & - & - & C1 & D1 & Euconulus alderi (Grays, 1840) & $\mathrm{pr}$ & $\mathrm{pr}$ \\
\hline & 2 & 20 & P4 & 2 & 1 & 1 & 0,75 & A3 & - & C1 & D1 & Helicella itala itala (Linnaeus, 1758) & $\mathrm{pr}$ & $\mathrm{pr}$ \\
\hline & 2 & 20 & $\mathrm{P} 4$ & 2 & 1 & 1 & 1 & - & - & C1 & D1 & Lauria cylindracea (Da Costa, 1778) & $\mathrm{pr}$ & $\mathrm{pr}$ \\
\hline & 2 & 20 & $\mathrm{P} 4$ & 2 & 1 & 1 & 0,6 & $\mathrm{~A} 2$ & - & C1 & D1 & Oxyloma elegans (Risso, 1826) & $\mathrm{pr}$ & $\mathrm{pr}$ \\
\hline & 2 & 20 & $\mathrm{P} 4$ & 2 & 1 & 1 & 1,5 & - & - & C1 & D1 & Pyramidula pusilla (Vallot, 1801) & $\mathrm{pr}$ & $\mathrm{pr}$ \\
\hline & 2 & 20 & $\mathrm{P} 4$ & 2 & 1 & 1 & 0,6 & $\mathrm{~A} 2$ & - & C1 & D1 & Succinea putris (Linnaeus, 1758) & $\mathrm{pr}$ & pr \\
\hline & 2 & 20 & $\mathrm{P} 4$ & 2 & 1 & 1 & 0,5 & $\mathrm{~A} 1$ & - & C1 & D1 & Trochulus hispidus (Linnaeus, 1758) & $\mathrm{pr}$ & $\mathrm{pr}$ \\
\hline & 2 & 20 & $\mathrm{P} 4$ & 2 & 1 & 1 & 0,5 & A1 & - & C1 & D1 & Trochulus plebeius (Draparnaud, 1805) & $\mathrm{pr}$ & $\mathrm{pr}$ \\
\hline & 2 & 20 & $\mathrm{P} 4$ & 2 & 1 & 1 & 1 & - & - & C1 & D1 & Truncatellina cylindrica (A. Férussac, 1807) & $\mathrm{pr}$ & $\mathrm{pr}$ \\
\hline & 2 & 20 & P4 & 2 & 1 & 1 & 1 & - & - & C1 & D1 & Vallonia excentrica Sterki, 1893 & $\mathrm{pr}$ & pr \\
\hline & 2 & 20 & $\mathrm{P} 4$ & 2 & 1 & 1 & 1 & - & - & C1 & D1 & Vallonia pulchella (O. F. Müller, 1774) & $\mathrm{pr}$ & $\mathrm{pr}$ \\
\hline & 2 & 20 & $\mathrm{P} 4$ & 2 & 1 & 1 & 1,33 & - & - & C1 & D1 & Vertigo antivertigo (Draparnaud, 1801) & $\mathrm{pr}$ & $\mathrm{pr}$ \\
\hline & 2 & 20 & P4 & 2 & 1 & 1 & 1 & - & - & C1 & D1 & Vertigo pusilla O. F. Müller, 1774 & $\mathrm{pr}$ & pr \\
\hline & 2 & 20 & $\mathrm{P} 4$ & 2 & 1 & 1 & 1 & - & - & C1 & D1 & Vitrea contracta (Westerlund, 1871) & $\mathrm{pr}$ & $\mathrm{pr}$ \\
\hline & 2 & 20 & $\mathrm{P} 4$ & 2 & 1 & 1 & 0,75 & A3 & - & C1 & D1 & Vitrina pellucida (O. F. Müller, 1774) & $\mathrm{pr}$ & pr \\
\hline & 1,5 & 21 & P4 & 1 & 1 & 1,5 & 1,5 & - & - & C1 & D1 & $\begin{array}{l}\text { Isognomostoma isognomostomos } \\
\text { (Schröter, 1784) }\end{array}$ & pr & pr \\
\hline & 1 & 22 & P4 & 1 & 1 & 1 & 1 & - & - & C1 & D1 & Abida secale (Draparnaud, 1801) & $\mathrm{pr}$ & $\mathrm{pr}$ \\
\hline & 1 & 22 & P4 & 1 & 1 & 1 & 1,5 & - & - & C1 & D1 & Acanthinula aculeata (O. F. Müller, 1774) & $\mathrm{pr}$ & $\mathrm{pr}$ \\
\hline & 1 & 22 & $\mathrm{P} 4$ & 1 & 1 & 1 & 0,75 & A3 & - & C1 & D1 & Aegopinella nitens (Michaud, 1831) & $\mathrm{pr}$ & $\mathrm{pr}$ \\
\hline & 1 & 22 & $\mathrm{P} 4$ & 1 & 1 & 1 & 0,75 & A3 & - & C1 & D1 & Aegopinella pura (Alder, 1830) & $\mathrm{pr}$ & pr \\
\hline & 1 & 22 & $\mathrm{P} 4$ & 1 & 1 & 1 & 1,5 & - & - & C1 & D1 & Arianta arbustorum (Linnaeus, 1758) & $\mathrm{pr}$ & $\mathrm{pr}$ \\
\hline & 1 & 22 & $\mathrm{P} 4$ & 1 & 1 & 1 & 1 & - & - & C1 & D1 & Carychium minimum O. F. Müller, 1774 & $\mathrm{pr}$ & $\mathrm{pr}$ \\
\hline & 1 & 22 & $\mathrm{P} 4$ & 1 & 1 & 1 & 1 & - & - & C1 & D1 & Carychium tridentatum (Risso, 1826) & $\mathrm{pr}$ & pr \\
\hline & 1 & 22 & $\mathrm{P} 4$ & 1 & 1 & 1 & 1,5 & - & - & C1 & D1 & Cecilioides acicula (O. F. Müller, 1774) & $\mathrm{pr}$ & $\mathrm{pr}$ \\
\hline & 1 & 22 & $\mathrm{P} 4$ & 1 & 1 & 1 & 1,5 & - & - & C1 & D1 & Cepaea hortensis (O. F. Müller, 1774) & $\mathrm{pr}$ & $\mathrm{pr}$ \\
\hline & 1 & 22 & P4 & 1 & 1 & 1 & 1,5 & - & - & C1 & D1 & Cepaea nemoralis (Linnaeus, 1758) & $\mathrm{pr}$ & $\mathrm{pr}$ \\
\hline & 1 & 22 & $\mathrm{P} 4$ & 1 & 1 & 1 & 1 & - & - & C1 & D1 & Clausilia bidentata (Strøm, 1765) & $\mathrm{pr}$ & pr \\
\hline & 1 & 22 & $\mathrm{P} 4$ & 1 & 1 & 1 & 1 & - & - & $\mathrm{C} 1$ & D1 & Clausilia rugosa parvula (A. Férussac, 1807) & $\mathrm{pr}$ & $\mathrm{pr}$ \\
\hline & 1 & 22 & $\mathrm{P} 4$ & 1 & 1 & 1 & 0,6 & $\mathrm{~A} 2$ & - & C1 & D1 & Cochlicopa lubrica (O. F. Müller, 1774) & $\mathrm{pr}$ & $\mathrm{pr}$ \\
\hline & 1 & 22 & P4 & 1 & 1 & 1 & 1,5 & - & - & C1 & D1 & Cochlodina laminata (Montagu, 1803) & $\mathrm{pr}$ & pr \\
\hline & 1 & 22 & P4 & 1 & 1 & 1 & 1,5 & - & - & C1 & D1 & $\begin{array}{l}\text { Cochlostoma septemspirale } \\
\text { (Razoumowsky, 1789) }\end{array}$ & $\mathrm{pr}$ & $\mathrm{pr}$ \\
\hline & 1 & 22 & P4 & 1 & 1 & 1 & 1,5 & - & - & C1 & D1 & Discus rotundatus (O. F. Müller, 1774) & $\mathrm{pr}$ & $\mathrm{pr}$ \\
\hline & 1 & 22 & $\mathrm{P} 4$ & 1 & 1 & 1 & 1 & - & - & $\mathrm{C} 1$ & D1 & Euconulus fulvus (O. F. Müller, 1774) & $\mathrm{pr}$ & $\mathrm{pr}$ \\
\hline & 1 & 22 & $\mathrm{P} 4$ & 1 & 1 & 1 & 1,5 & - & - & C1 & D1 & Fruticicola fruticum (O. F. Müller, 1774) & $\mathrm{pr}$ & $\mathrm{pr}$ \\
\hline & 1 & 22 & $\mathrm{P} 4$ & 1 & 1 & 1 & 1,5 & - & - & C1 & D1 & Helicigona lapicida (Linnaeus, 1758) & $\mathrm{pr}$ & pr \\
\hline & 1 & 22 & $\mathrm{P} 4$ & 1 & 1 & 1 & 1,5 & - & - & $\mathrm{C} 1$ & D1 & Helicodonta obvoluta (O. F. Müller, 1774) & $\mathrm{pr}$ & $\mathrm{pr}$ \\
\hline & 1 & 22 & $\mathrm{P} 4$ & 1 & 1 & 1 & 1,5 & - & - & C1 & D1 & Merdigera obscura (O. F. Müller, 1774) & $\mathrm{pr}$ & $\mathrm{pr}$ \\
\hline & 1 & 22 & $\mathrm{P} 4$ & 1 & 1 & 1 & 1,5 & - & - & C1 & D1 & Monachoides incarnatus (O. F. Müller, 1774) & $\mathrm{pr}$ & $\mathrm{pr}$ \\
\hline & 1 & 22 & P4 & 1 & 1 & 1 & 0,75 & A3 & - & C1 & D1 & Nesovitrea hammonis (Strøm, 1765) & $\mathrm{pr}$ & pr \\
\hline & 1 & 22 & $\mathrm{P} 4$ & 1 & 1 & 1 & 0,75 & A3 & - & C1 & D1 & Oxychilus cellarius (O. F. Müller, 1774) & $\mathrm{pr}$ & pr \\
\hline & 1 & 22 & $\mathrm{P} 4$ & 1 & 1 & 1 & 1 & - & - & C1 & D1 & Pomatias elegans (O. F. Müller, 1774) & $\mathrm{pr}$ & $\mathrm{pr}$ \\
\hline & 1 & 22 & $\mathrm{P} 4$ & 1 & 1 & 1 & 1 & - & - & C1 & D1 & Punctum pygmaeum (Draparnaud, 1801) & $\mathrm{pr}$ & $\mathrm{pr}$ \\
\hline & 1 & 22 & $\mathrm{P} 4$ & 1 & 1 & 1 & 1 & - & - & C1 & D1 & Pupilla muscorum (Linnaeus, 1758) & $\mathrm{pr}$ & $\mathrm{pr}$ \\
\hline & 1 & 22 & $\mathrm{P} 4$ & 1 & 1 & 1 & 1 & - & - & C1 & D1 & Vallonia costata (O. F. Müller, 1774) & $\mathrm{pr}$ & $\mathrm{pr}$ \\
\hline & 1 & 22 & $\mathrm{P} 4$ & 1 & 1 & 1 & 1 & - & - & C1 & D1 & Vertigo pygmaea (Draparnaud, 1801) & $\mathrm{pr}$ & $\mathrm{pr}$ \\
\hline & 1 & 22 & $\mathrm{P} 4$ & 1 & 1 & 1 & 1 & - & - & C1 & D1 & Vitrea crystallina (O. F. Müller, 1774) & $\mathrm{pr}$ & $\mathrm{pr}$ \\
\hline & 1 & 22 & P4 & 1 & 1 & 1 & 1,5 & - & - & C1 & D1 & Zonitoides nitidus (O. F. Müller, 1774) & $\mathrm{pr}$ & $\mathrm{pr}$ \\
\hline & - & - & NE & NE & NE & NE & - & - & - & - & - & Backeljaia gigaxii (L. Pfeiffer, 1847) & $\mathrm{pr}$ & $\mathrm{pr}$ \\
\hline & - & - & NE & NE & NE & NE & - & - & - & - & - & Cernuella neglecta (Draparnaud, 1805) & $\mathrm{pr}$ & $\mathrm{pr}$ \\
\hline & - & - & NE & NE & $\mathrm{NE}$ & NE & - & - & - & - & - & Cernuella virgata (da Costa, 1778) & $a b$ & $\mathrm{pr}$ \\
\hline & - & - & NE & NE & NE & NE & - & - & - & - & - & Cornu aspersum (O. F. Müller, 1774) & $\mathrm{pr}$ & pr \\
\hline
\end{tabular}


ANNEXE 2. - Suite.

\begin{tabular}{|c|c|c|c|c|c|c|c|c|c|c|c|c|c|c|}
\hline \multirow[b]{2}{*}{ Groupes } & \multirow[b]{2}{*}{ SG } & \multirow[b]{2}{*}{ Rang } & \multirow[b]{2}{*}{ Cat } & \multicolumn{3}{|c|}{ Critères } & \multicolumn{5}{|c|}{ Actions compensatoires } & \multirow[b]{2}{*}{ Nom valide TAXREF v13.0 } & \multicolumn{2}{|c|}{ Régions } \\
\hline & & & & $\mathbf{R}$ & $\mathbf{V}$ & $\mathbf{R R}$ & $\mathrm{M} / \mathrm{H}$ & A & B & C & D & & BFC & GE \\
\hline & - & - & $\mathrm{NE}$ & $\mathrm{NE}$ & NE & NE & - & - & - & - & - & Helix lucorum Linnaeus, 1758 & $\mathrm{pr}$ & $\mathrm{pr}$ \\
\hline & - & - & NE & NE & NE & NE & - & - & - & - & - & Helix pomatia Linnaeus, 1758 & $\mathrm{pr}$ & pr \\
\hline & - & - & NE & NE & NE & NE & - & - & - & - & - & Hygromia cinctella (Draparnaud, 1801) & $\mathrm{pr}$ & pr \\
\hline & - & - & NE & NE & NE & NE & - & - & - & - & - & Monacha cantiana (Montagu, 1803) & $\mathrm{pr}$ & $\mathrm{pr}$ \\
\hline & - & - & NE & $\mathrm{NE}$ & NE & NE & - & - & - & - & - & Monacha cartusiana (O. F. Müller, 1774) & $\mathrm{pr}$ & $\mathrm{pr}$ \\
\hline & - & - & NE & NE & NE & NE & - & - & - & - & - & Monacha cemenelea (Risso, 1826) & pr & $a b$ \\
\hline & - & - & NE & NE & NE & NE & - & - & - & - & - & Paralaoma servilis (Shuttleworth, 1852) & $a b$ & pr \\
\hline & - & - & NE & NE & NE & NE & - & - & - & - & - & Testacella haliotidea Lamarck, 1801 & $\mathrm{pr}$ & $\mathrm{pr}$ \\
\hline & - & - & NE & NE & NE & NE & - & - & - & - & - & Xerolenta obvia (Menke, 1828) & $a b$ & $\mathrm{pr}$ \\
\hline & - & - & NE & NE & $\mathrm{NE}$ & NE & - & - & - & - & - & Xeropicta derbentina (Krynicki, 1836) & $\mathrm{pr}$ & $a b$ \\
\hline & - & - & NE & NE & NE & NE & - & - & - & - & - & Xeroplexa intersecta (Poiret, 1801) & $\mathrm{pr}$ & $a b$ \\
\hline
\end{tabular}

